\title{
Kingdom of Lesotho: Request for a Three-Year Arrangement Extended Fund Facility Arrangement - Staff Report; Staff Supplement; Press Release on the Executive Board Discussion; Statement by the Executive Director for the Kingdom of Lesotho
}

In the context of the request for a three-year arrangement extended fund facility arrangement, the following documents have been released and are included in this package:

- $\quad$ The staff report for the Request for a Three-Year Arrangement Extended Fund Facility Arrangement, prepared by a staff team of the IMF, following discussions that ended on March 24, 2010, with the officials of Lesotho on economic developments and policies. Based on information available at the time of these discussions, the staff report was completed on May 17, 2010. The views expressed in the staff report are those of the staff team and do not necessarily reflect the views of the Executive Board of the IMF.

- $\quad$ A supplement on the joint IMF/World Bank debt sustainability analysis.

- $\quad$ A Press Release summarizing the views of the Executive Board as expressed during its June 2, 2010 discussion of the staff report that completed the request and/or review.

- $\quad$ A statement by the Executive Director for the Kingdom of Lesotho.

The documents listed below have been or will be separately released.

Letter of Intent sent to the IMF by the authorities of Lesotho*

Memorandum of Economic and Financial Policies by the authorities of

Lesotho*

Technical Memorandum of Understanding*

*Also included in Staff Report

The policy of publication of staff reports and other documents allows for the deletion of market-sensitive information.

Copies of this report are available to the public from

International Monetary Fund • Publication Services

$70019^{\text {th }}$ Street, N.W. • Washington, D.C. 20431

Telephone: (202) 623-7430 • Telefax: (202) 623-7201

E-mail: publications@imf.org Internet: http://www.imf.org

\section{International Monetary Fund Washington, D.C.}


INTERNATIONAL MONETARY FUND

KINGDOM OF LESOTHO

\section{Request for a Three-Year Arrangement Under the Extended Credit Facility}

Prepared by the African Department in Consultation with Other Departments

Approved by Domenico Fanizza and Tom Dorsey

May 17, 2010

- Mission: March 10-24, 2010. The mission met with Finance and Development

Planning Minister Thahane, Central Bank of Lesotho Governor Senaoana, other senior government officials, and representatives of the donor community. Discussions were concluded on April 26, 2010.

- $\quad$ Team: Messrs. Thugge (head), Pastor and Benicio, and Miss Morgan (all AFR). Ms. Lephoto (OED) also attended.

- Extended Credit Facility arrangement: The authorities are requesting a three-year ECF arrangement with total access of SDR 41.88 million (120 percent of quota) to support their medium-term adjustment program and help reduce balance of payments risks in light of the significant reduction in SACU revenues.

- Data Issues: Macroeconomic data have some shortcomings, but are broadly adequate for surveillance and program monitoring.

- Publication: The authorities have consented to publication of the staff report and the program documents. 


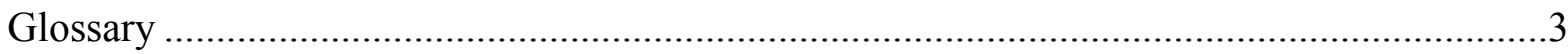

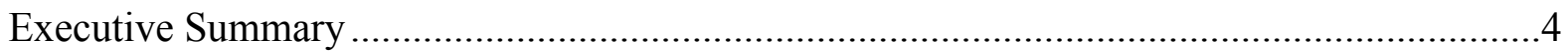

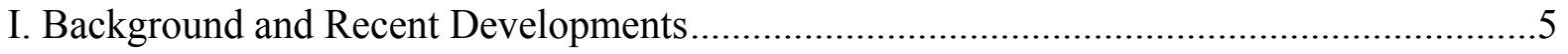

II. The three-year Arrangement Under the Extended Credit Facility (ECF) ..........................

A. Medium-Term Macroeconomic Framework for 2010-13 ...................................

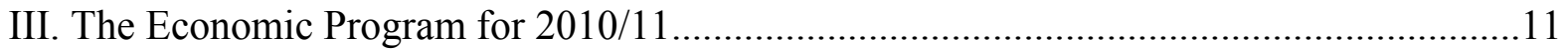

A. Fiscal Consolidation........................................................................................ 11

B. Structural Reforms to Increase Productivity and External Competitiveness ..........14

C. Financial Sector Strengthening to Enhance Access to Financial Services and Support Economic Development...................................................................15

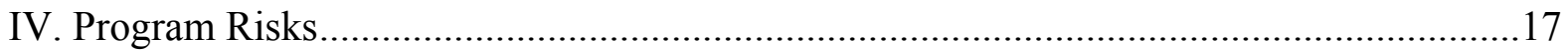

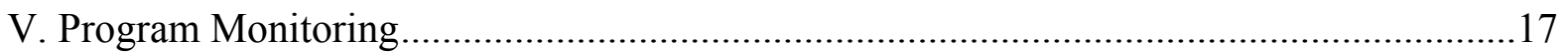

A. Program Monitoring and Conditionality ........................................................ 17

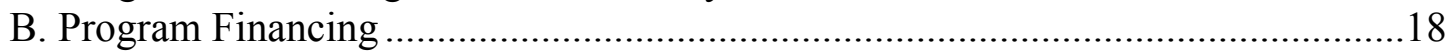

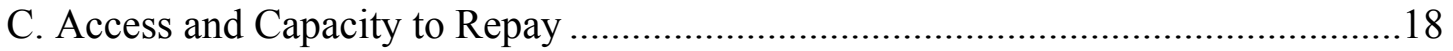

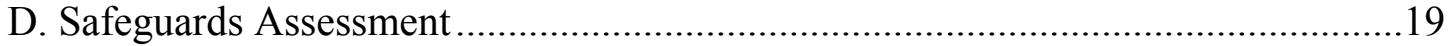

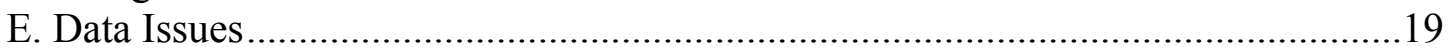

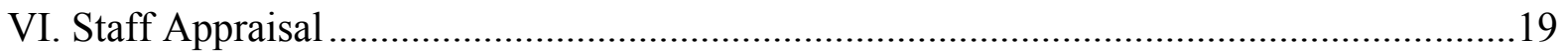

Tables

1. Selected Economic and Financial Indicators, 2006-2015 …........................................22

2. Central Government Operations, 2007/08-2015/16 (In millions of maloti) .....................23

2a. Central Government Operations 2007/08-2015/16 (In percent of GDP) .......................24

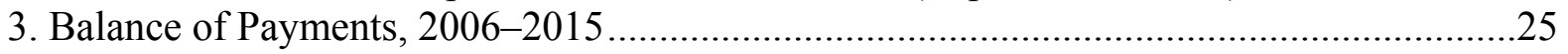

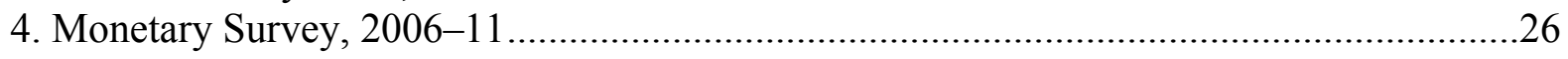

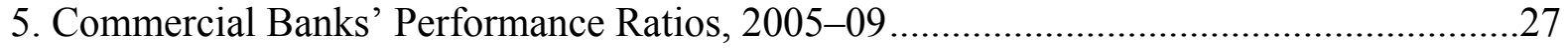

6. External Financing Requirements and Sources, 2010-2013 ........................................28

7. Schedule of ECF Disbursements and Reviews, 2010-13 ..............................................29

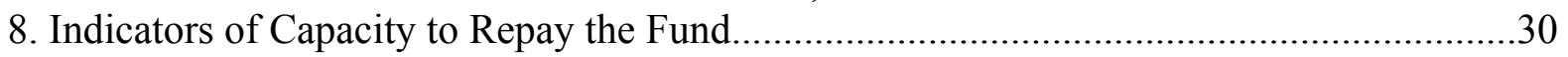

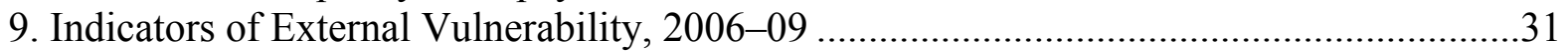

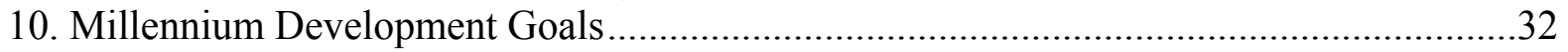

Box

1. The Metolong Dam and the Lesotho Highlands Water Project...................................... 12

Attachments

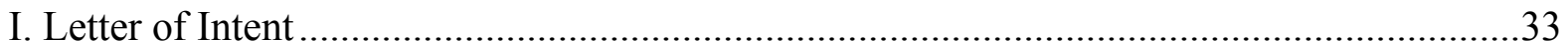

II. Memorandum of Economic and Financial Policies 2010-2013 ...................................34

III. Technical Memorandum of Understanding .............................................................46 


\section{GLOSSARY}

$\begin{array}{ll}\text { AfDB } & \text { African Development Bank } \\ \text { CBL } & \text { Central Bank of Lesotho } \\ \text { CMA } & \text { Common Monetary Area } \\ \text { CPI } & \text { Consumer Price Index } \\ \text { DSA } & \text { Debt Sustainability Analysis } \\ \text { ECF } & \text { Extended Credit Facility } \\ \text { EIB } & \text { European Investment Bank } \\ \text { EU } & \text { European Union } \\ \text { FAD } & \text { Fiscal Affairs Department } \\ \text { FIA } & \text { Financial Institutions Act } \\ \text { FDI } & \text { Foreign Direct Investment } \\ \text { GDP } & \text { Gross Domestic Product } \\ \text { IFMIS } & \text { Integrated Financial Management Information System } \\ \text { LHDA } & \text { Lesotho Highlands Development Authority } \\ \text { LHWP } & \text { Lesotho Highlands Water Project } \\ \text { LNDC } & \text { Lesotho National Development Corporation } \\ \text { LRA } & \text { Lesotho Revenue Authority } \\ \text { MCC } & \text { Millennium Challenge Corporation } \\ \text { M } & \text { Maloti (plural for the Loti, Lesotho's currency) } \\ \text { MEFP } & \text { Memorandum of Economic and Financial Policies } \\ \text { MoFDP } & \text { Ministry of Finance and Development Planning } \\ \text { MDGs } & \text { Millennium Development Goals } \\ \text { NBFIs } & \text { Non-bank Financial Institutions } \\ \text { PAC } & \text { Project Appraisal Committee } \\ \text { PEM } & \text { Public Expenditure Management } \\ \text { PFM } & \text { Public Financial Management } \\ \text { SACU } & \text { Southern African Customs Union } \\ \text { SACCOs } & \text { Savings and Credit Cooperatives } \\ \text { SADC } & \text { Southern African Development Community } \\ \text { TMU } & \text { Technical Memorandum of Understanding } \\ & \end{array}$




\section{EXECUTIVE SUMMARY}

After several years of macroeconomic stability, Lesotho's economic performance deteriorated in 2009. Real GDP decelerated in the wake of reduced demand for the main exports of textiles and diamonds, and significant decline in workers' remittances from South Africa. Persistent increases in public expenditure in the past three years led to a deterioration of the fiscal balance, which shifted into deficit in 2009/10, after five consecutive years of surpluses. The external current account also shifted into deficit, reflecting lower export earnings and income receipts.

Starting from 2010/11, Lesotho will face large fiscal and external imbalances. The global crisis has resulted in a significant decline in SACU revenues, which account for around 60 percent of tax revenue. SACU revenues are projected to decline by some 23 percent of GDP during 2010/11-2011/12, mainly due to lower imports by South Africa. As a result, Lesotho's fiscal and external balances will deteriorate significantly, and remain vulnerable to downside risks. While SACU revenues are projected to recover somewhat, the authorities expect that these revenues will stabilize at levels well below trend.

The main policy challenges for the authorities is to respond to lower SACU revenues, by undertaking key fiscal adjustments to restore macroeconomic stability as a necessary condition for raising Lesotho's growth potential to reduce poverty. Fiscal consolidation will be key to restoring macroeconomic stability, complemented by fast-tracking of structural reforms.

The authorities have adopted a comprehensive medium-term macroeconomic program to address these challenges, in support of which they are requesting Fund support under an ECF arrangement. The program is designed to restore fiscal and external sustainability, achieve broad-based growth and strengthen the financial sector. Key reform measures to be pursued are: (a) containing expenditure to levels consistent with sustainable revenue flows, while safeguarding social spending for the poor and vulnerable groups; (b) further strengthening of public expenditure and financial management; (c) improving the business climate to facilitate private sector development; and (d) strengthening the regulatory framework for the financial sector and deepening financial intermediation. The policies are consistent with the authorities' Interim National Development Framework.

Staff supports the authorities' request for a three-year ECF arrangement in the amount of SDR 41.88 million (120 percent of quota). Fund financing would support Lesotho's efforts to restore macroeconomic stability and help reduce balance of payments risks. 


\section{BACKGROUND AND RECENT DEVELOPMENTS}

1. Lesotho experienced strong macroeconomic performance during 2006-08, supported by favorable external conditions. Real GDP growth averaged 4.5 percent, driven by the construction, textile and mining sectors. Sizeable revenues from the Southern African Customs Union (SACU) resulted in large fiscal and current account surpluses, and boosted gross international reserves. However, gains in reducing poverty and income inequality remained limited, reflecting delays in implementing key structural reforms to generate broadbased growth. In addition, the HIV/AIDS pandemic negatively impacted socioeconomic development and continues to threaten the achievement of the MDGs.

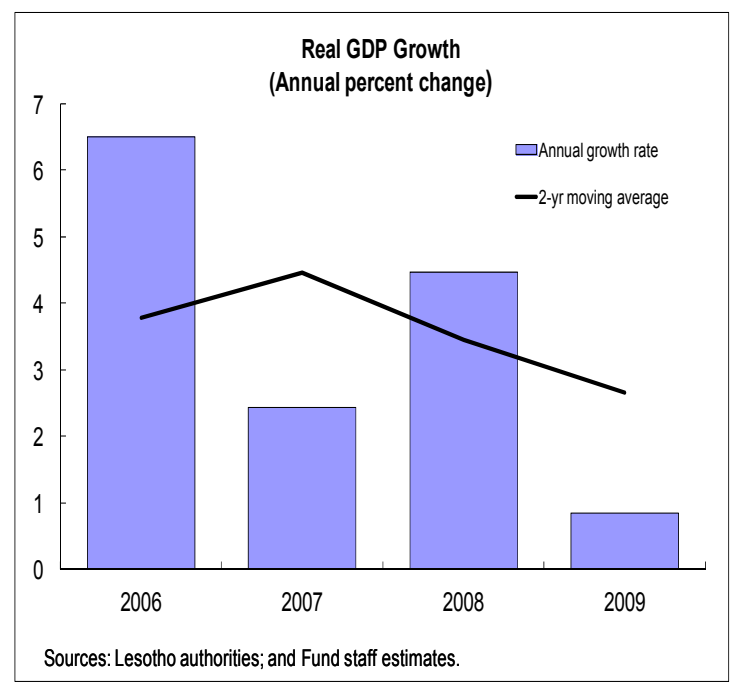

2. Macroeconomic conditions deteriorated sharply in 2009, reflecting the adverse effects of the global economic crisis and a loosening of fiscal policy. Economic growth slowed to 0.9 percent from 4.5 percent in 2008 , due to reduced demand for diamond and textile exports, while workers' remittances fell sharply. On the fiscal front, expenditure rose rapidly in 2009/10 to reach an unprecedented level of 69 percent of GDP. For the first time in five years, the fiscal position shifted into a deficit of 2.7 percent of GDP in 2009/10, compared with surpluses averaging 8.8 percent of GDP during 2006/07-2008/09.

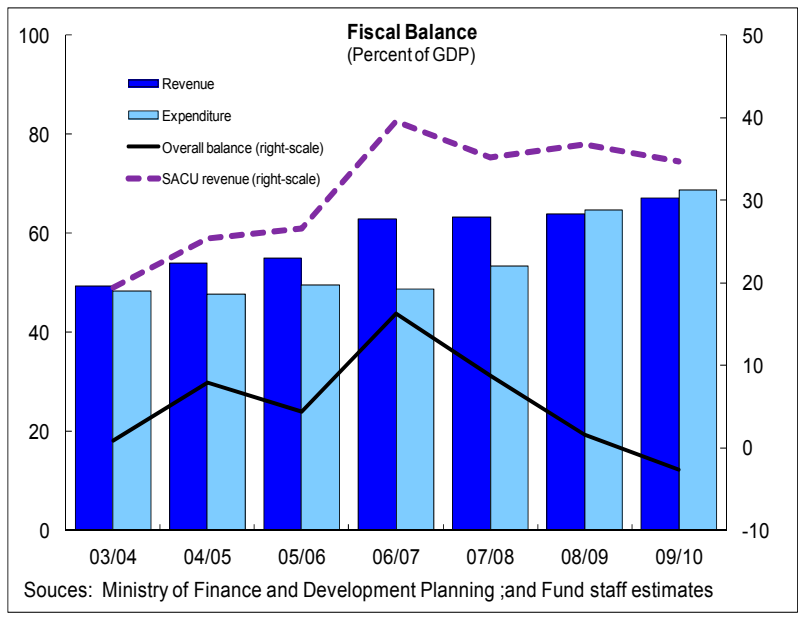

3. The external current account balance also shifted into deficit position in 2009. After several years of large surpluses averaging 9.4 percent of GDP during 2006-2008, the current account position shifted into a deficit of 0.3 percent of GDP in 2009, mainly reflecting lower export earnings and worker's remittances. Nonetheless, capital inflows during 2009 (mainly MCC grants, the SDR allocation and foreign direct investment by Philips Electronics) facilitated a build-up of international reserves to the equivalent of 7.7 months of imports. 

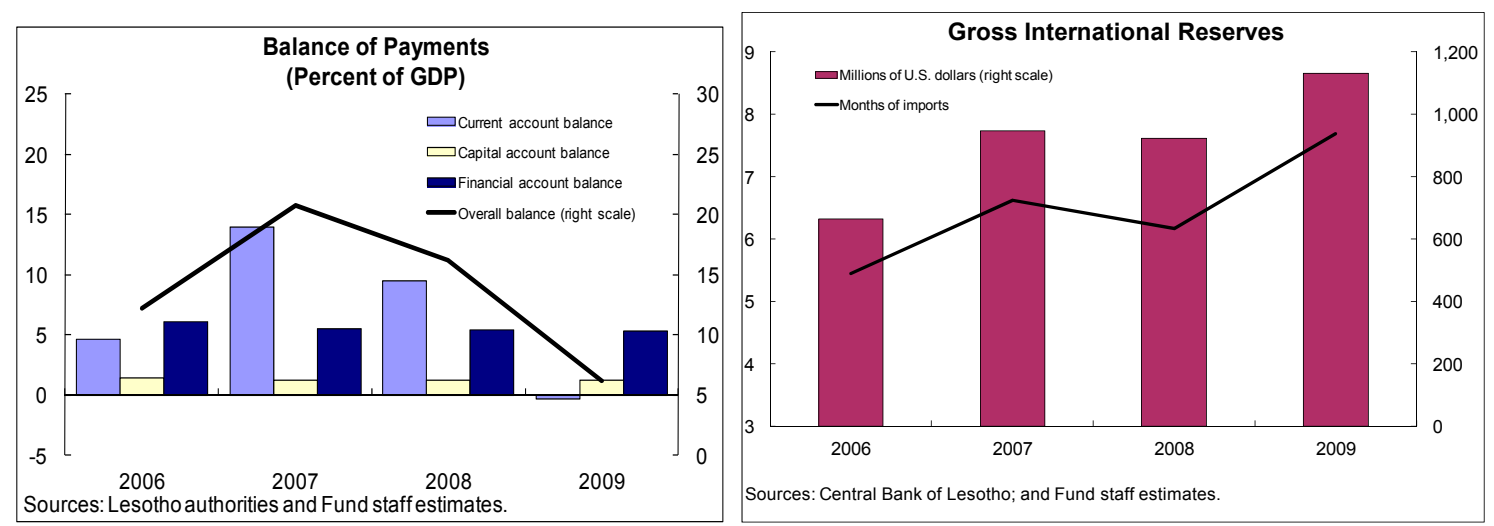

4. Monetary policy remained focused on supporting the exchange rate peg and price stability. In December 2009, the Central Bank of Lesotho (CBL) raised its target range for net international reserves from US\$500-550 million to US\$650-700 million. The 12-month inflation slowed to 4.2 percent at end-December 2009, compared with 10.6 percent at end-December 2008, largely reflecting declining prices for food which represents a large share of the consumer price index basket. With the loti pegged to the rand, inflation and interest rates developments in Lesotho broadly mirror those of South Africa.
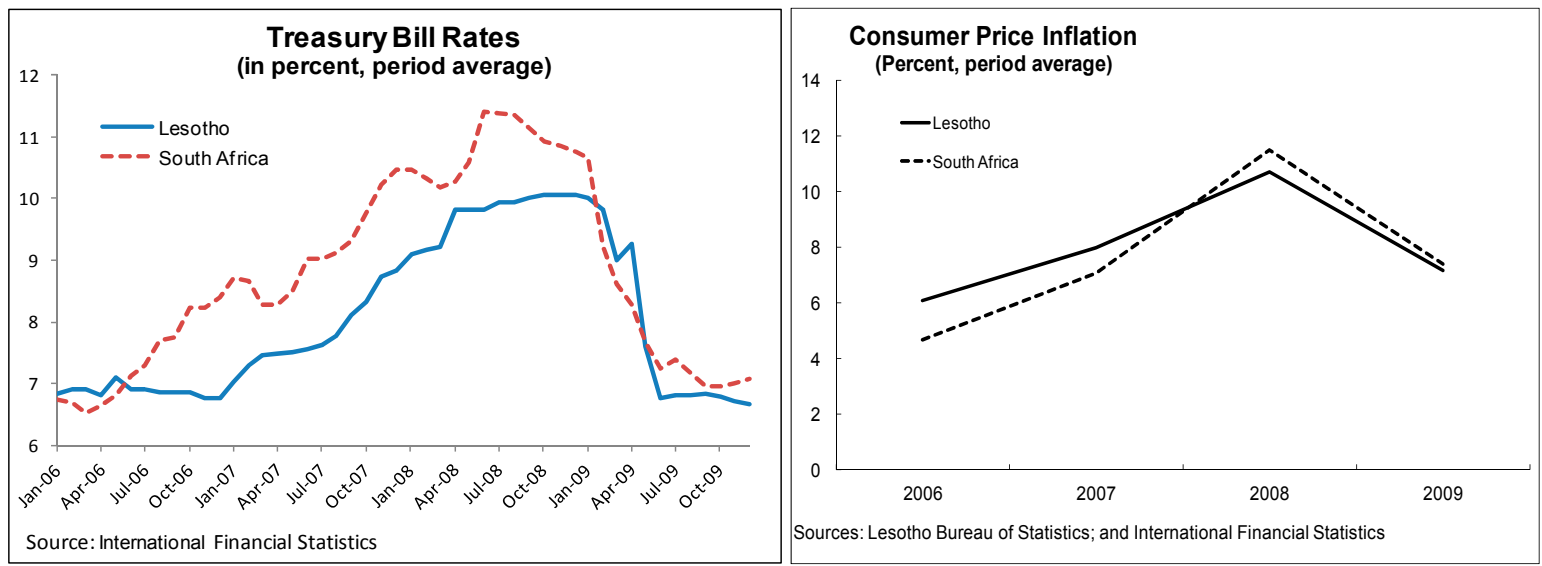

5. The financial sector weathered the global financial crisis relatively well. Banks are well capitalized, liquid and profitable. However, the sector remains vulnerable from weakly supervised nonbank financial institutions (NBFIs) and the potential for reemergence of Ponzi schemes. The CBL is making progress in strengthening the regulatory framework. 

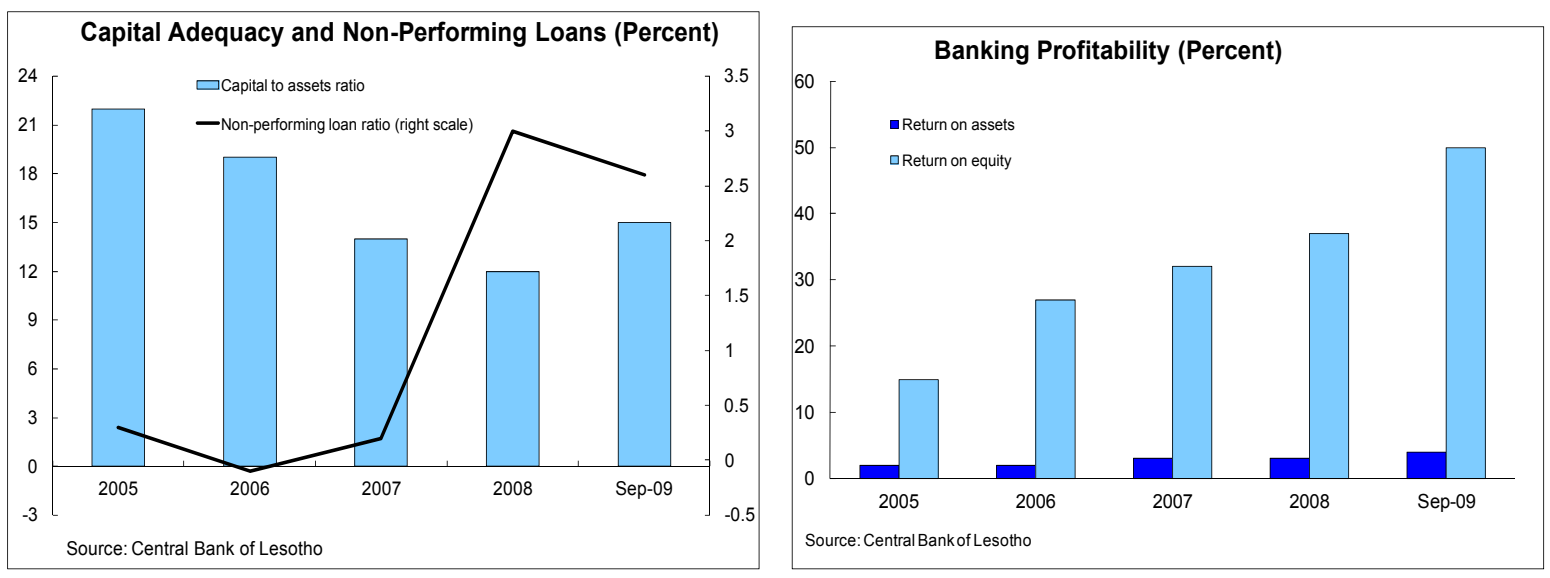

\section{THE THREE-YEAR ARRANGEMENT UNDER THE EXTENDED CREDIT FACILITY (ECF)}

\section{A. Medium-Term Macroeconomic Framework for 2010-13}

6. Lesotho faces serious macro-economic challenges over the medium term. SACU revenues are projected to decline by some 23 percent of GDP during 2010/11-2011/12 because of lower imports by South Africa, due to the global economic crisis. ${ }^{1}$ In the absence of policy adjustment measures, the reduction in SACU revenues would result in an even sharper deterioration in the fiscal deficit (excluding Metolong dam) to an average of about 23 percent of GDP in 2010/11-2011/12. ${ }^{2}$ Although SACU revenues are projected to recover in line with expected economic expansion in South Africa and the completion of repayments to the Common Revenue Pool, they are expected to stabilize at around 20 percent of GDP in the medium term, well below the average of 37 percent of GDP during 2006/07-2008/09. Moreover, risks remain for further decline in SACU revenues from a change in the revenuesharing formula, a reduction in the Common External Tariff rates due to trade liberalization, and the creation of a SADC customs union. Strong fiscal adjustment will therefore be required in order to restore fiscal, debt and external sustainability.

\section{To address these challenges, the authorities have adopted a medium-term} macroeconomic program designed to restore fiscal and external sustainability, achieve sustained broad-based growth for poverty reduction, and strengthen the financial sector. In support of these objectives, the authorities plan to implement measures to:

(a) contain public expenditure while protecting the poor and vulnerable groups;

(b) strengthen non-SACU revenues; (c) further strengthen public financial management to

\footnotetext{
${ }^{1}$ SACU repayments to the Common Revenue Pool, owing to overpayments for 2008/09 and 2009/10, account for about 5 percent of GDP of total reduction in SACU revenues in 2010/11 and about 9 percent of GDP in $2011 / 12$.

${ }^{2}$ The reference to the deficit excluding the Metolong dam is to reflect the fact that these capital expenditures are one-off and drop out of the medium-term fiscal framework after 2012/13.
} 
improve spending efficiency and public service delivery; (d) improve the business climate to facilitate private sector expansion; and (e) strengthen the regulatory framework for the financial sector while enhancing access to financial services, particularly in rural areas (MEFP ๆ 7-15).

\begin{tabular}{|c|c|c|c|c|c|c|c|c|}
\hline \multicolumn{9}{|c|}{ Text Table 1: Lesotho: Medium-Term Key Economic Indicators, 2008-2015 } \\
\hline & \multirow[b]{2}{*}{2008} & \multirow{2}{*}{$\begin{array}{r}\text { Est. } \\
2009 \\
\end{array}$} & & \multicolumn{2}{|c|}{ Projections } & \multirow[b]{2}{*}{2013} & \multirow[b]{2}{*}{2014} & \multirow[b]{2}{*}{2015} \\
\hline & & & 2010 & 2011 & 2012 & & & \\
\hline \multicolumn{9}{|c|}{ (Annual percentage change, unless otherwise indicated) } \\
\hline Real GDP Growth & 4.5 & 0.9 & 5.6 & 3.3 & 5.3 & 3.0 & 5.0 & 12.1 \\
\hline Consumer price index (eop) & 10.6 & 4.2 & 5.8 & 5.6 & 5.5 & 4.6 & 4.6 & 5.0 \\
\hline \multicolumn{9}{|c|}{ (In percent of GDP, unless otherwise indicated) } \\
\hline Overall fiscal balance (incl. grants) $v$ & 1.6 & -2.7 & -19.0 & -17.9 & -6.8 & -2.4 & -0.8 & 1.3 \\
\hline Overall fiscal balance (incl. grants, excl. M etolong) $v$ & $\ldots$ & & -15.3 & -14.2 & -3.1 & -1.8 & -0.4 & 1.7 \\
\hline Government debt $v$ & 52.1 & 40.2 & 45.2 & 49.8 & 53.1 & 55.3 & 54.4 & 52.4 \\
\hline External debt-service ratio $2 /$ & 3.9 & 4.3 & 4.4 & 4.5 & 4.7 & 4.8 & 4.6 & 4.5 \\
\hline Current account balance (incl. official transfers) & 9.5 & -0.3 & -22.0 & -22.5 & -18.8 & -8.9 & -7.6 & -12.0 \\
\hline Gross official reserves (US\$ millions) & 923.1 & 1131.0 & 854.5 & 651.0 & 638.7 & 700.7 & 740.3 & 775.8 \\
\hline Months of imports of goods and services & 6.2 & 7.7 & 5.2 & 4.0 & 3.7 & 4.1 & 4.3 & 4.5 \\
\hline $\begin{array}{l}\text { Sources: Lesotho authorities; and IMF staff estimates and } \\
\text { 1 Fiscal data are reported on a fiscal year basis; fiscal year } \\
\text { 2/ Percent of exports of goods and nonfactor services. }\end{array}$ & $\begin{array}{l}\text { ons. } \\
\text { in Apri }\end{array}$ & & & & & & & \\
\hline
\end{tabular}

8. The proposed medium-term fiscal adjustment path would curtail nonpriority expenditure to achieve a level of expenditure that is consistent with lower SACU revenues. Specific measures will include containing the growth of the wage bill and of goods and services (MEFP \ 10-11). These expenditure reductions, together with some recovery in SACU revenues, would result in a narrowing of the fiscal deficit (excluding Metolong) from about 15.3 percent of GDP in 2010/11 to a small surplus by $2015 / 16$. The authorities plan to finance the deficit through a combination of

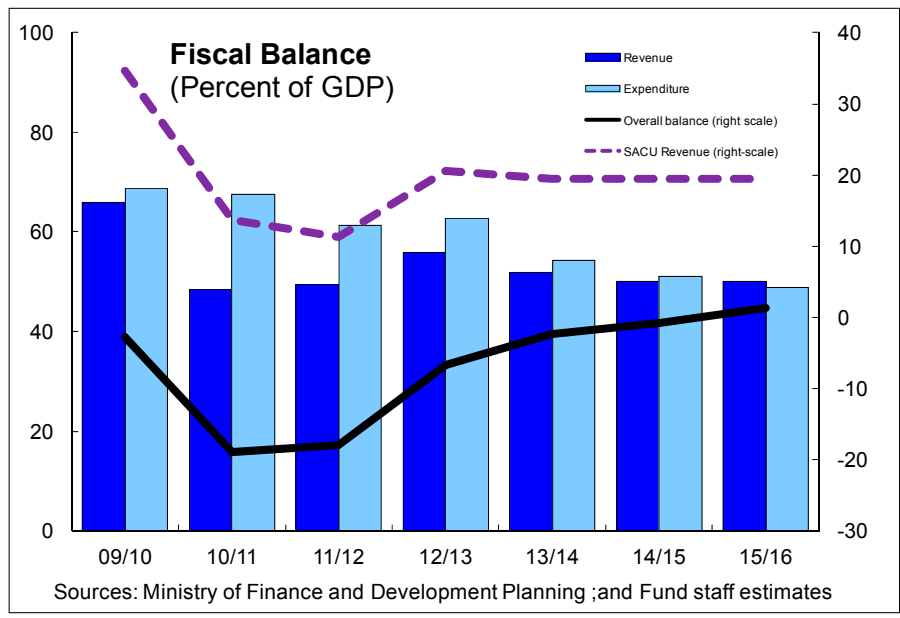
drawdown in deposits at the CBL, budget support grants and new loans from development partners, and by issuing domestic bonds. Given the direct impact of domestic financing on international reserves, the authorities are committed to a cautious drawdown of deposits to protect the credibility of the exchange rate peg. 


\begin{tabular}{|c|c|c|c|c|c|c|c|c|}
\hline \multirow[b]{3}{*}{ Total revenue and grants } & \multirow{2}{*}{$\begin{array}{c}\text { Est. } \\
2009 / 10\end{array}$} & \multirow{2}{*}{\begin{tabular}{|l} 
Budget \\
$2010 / 11$
\end{tabular}} & \multicolumn{6}{|c|}{ Projections } \\
\hline & & & $2010 / 11$ & 201112 & $2012 / 13$ & $2013 / 14$ & $2014 / 15$ & $2015 / 16$ \\
\hline & 66.0 & 49.8 & 48.5 & 49.5 & 56.0 & 52.0 & 50.1 & 50.1 \\
\hline Revenue & 61.6 & 41.0 & 40.9 & 38.7 & 47.8 & 46.6 & 46.6 & 46.6 \\
\hline SACU revenue & 34.7 & 13.7 & 13.7 & 11.4 & 20.5 & 19.4 & 19.4 & 19.4 \\
\hline Non-SACU Revenue & 26.8 & 27.3 & 27.2 & 27.3 & 27.3 & 27.2 & 27.2 & 27.3 \\
\hline Total expenditure and net lending & 68.7 & 69.2 & 67.5 & 67.5 & 62.7 & 54.3 & 51.0 & 48.8 \\
\hline Current expenditure & 51.3 & 43.9 & 43.9 & 41.1 & 40.0 & 38.2 & 37.5 & 35.9 \\
\hline o/w Wages and salaries & 18.1 & 17.0 & 17.0 & 15.1 & 13.7 & 13.7 & 13.7 & 13.7 \\
\hline o/w Goods and services & ₹.. & 11.2 & 11.2 & 10.6 & 0.1 & 9.9 & 9.7 & 8.9 \\
\hline Capital expenditure & 16.0 & 25.4 & 23.6 & 26.4 & 22.8 & 16.1 & 13.5 & 12.9 \\
\hline Overall balance, after grants & -2.7 & -19.5 & -19.0 & -17.9 & -6.8 & -2.4 & -0.8 & 1.3 \\
\hline Overall balance (after grant, excl. M etolong) & $\ldots$ & -15.7 & -15.3 & -14.2 & -3.1 & -1.8 & -0.4 & 1.7 \\
\hline $\begin{array}{l}\text { Non--SA CU balance } 2 / \\
\text { Primary balance }\end{array}$ & $\begin{array}{l}-37.4 \\
-1.9\end{array}$ & $\begin{array}{l}-33.2 \\
-18.3\end{array}$ & $\begin{array}{l}-32.7 \\
-18.1\end{array}$ & $\begin{array}{l}-29.3 \\
-16.9\end{array}$ & $\begin{array}{l}-27.3 \\
-5.5\end{array}$ & $\begin{array}{l}-21.7 \\
-1.0\end{array}$ & $\begin{array}{l}-20.2 \\
0.5\end{array}$ & $\begin{array}{l}-18.1 \\
2.5\end{array}$ \\
\hline \multicolumn{9}{|c|}{ Sources: M inistry of Finance and Develo pment Planning, and Fund staff estimates and projections. } \\
\hline \multicolumn{9}{|l|}{$\checkmark$ The fiscal year runs from A pril $1 \mathrm{M}$ arch 31.} \\
\hline
\end{tabular}

\section{Reflecting developments in the fiscal position, the external current account} deficit is projected to widen to an average of 21.0 percent of GDP during 2010-2012, mainly because of the loss of SACU revenues. Over the medium term, as fiscal consolidation takes hold and there is a recovery of SACU revenues, the current account deficit will narrow from 22 percent of GDP in 2010 to 12 percent of GDP by $2015 .{ }^{3}$ With the proposed Fund assistance, the level of international reserves is projected to remain above the CBL's target for supporting the exchange rate peg.

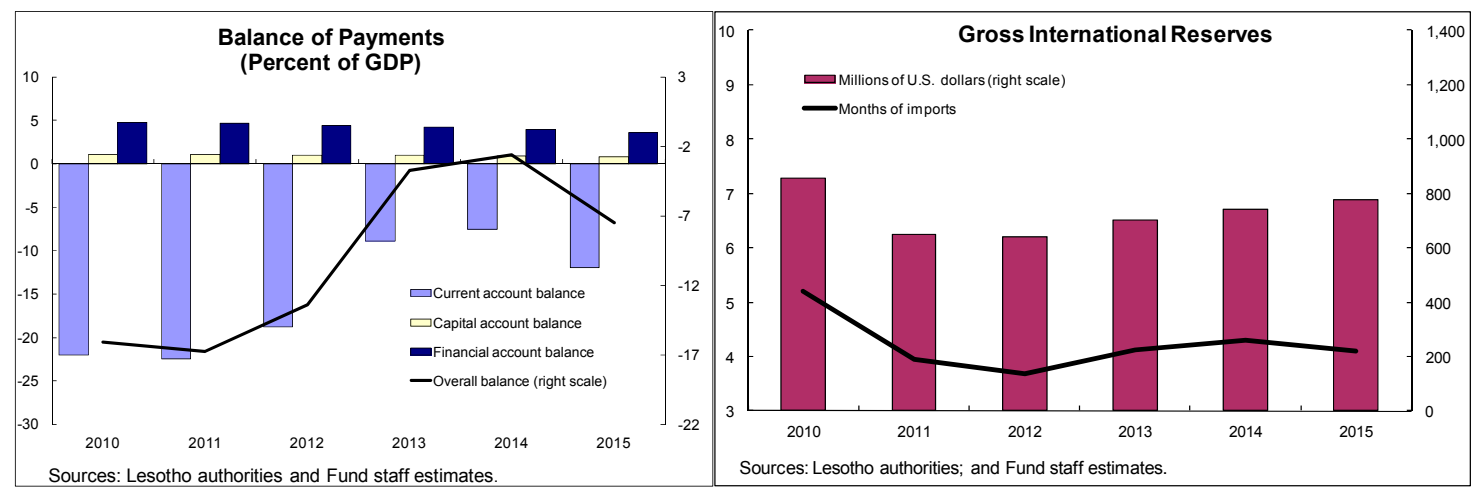

\footnotetext{
${ }^{3}$ Excluding Metolong and LHWPII, the current account deficit would narrow from 20 percent of GDP in 2010 to 4 percent of GDP in 2015 .
} 
10. The authorities' program envisages economic activity to recover gradually over the medium term. Real GDP growth is projected to average 4.3 percent during 2010-13, predicated on substantial increases in public infrastructure investment, including construction of the Metolong dam and the start-up of the second phase of the Lesotho Highlands Water Project (LHWPII) (Box 1), and the expansion of private sector activity, particularly in mining.

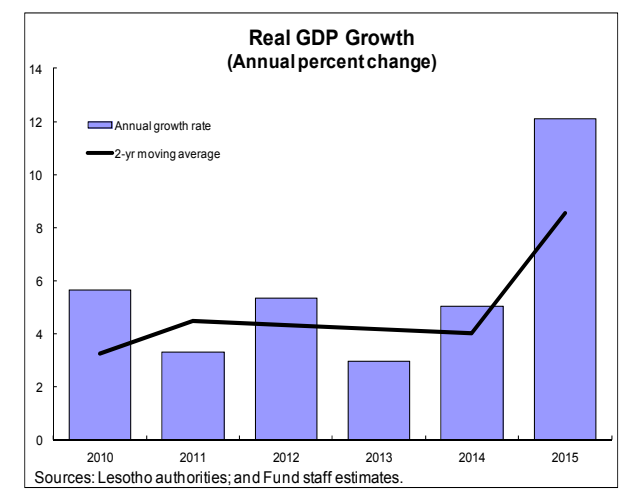

11. Over the medium term, public expenditure and financial management reforms will focus on strengthening budget preparation, execution and accountability. Key measures include introduction and publication of a consolidated medium-term expenditure framework, regular reports on budget performance, introduction of a cash management system, and addressing deficiencies in the Integrated Financial Management Information System (IFMIS). These measures are based on the recommendations made by a March 2010 Fiscal Affairs Department FAD TA mission.

\section{Implementing structural reforms remains central to achieving Lesotho's} medium-term goals of sustained broad-based growth for poverty reduction. The authorities' medium-term program includes measures to reduce infrastructure constraints, strengthen institutional capacity and the legal and regulatory framework, and to enhance property rights and land reform. These measures are considered critical for improving the business climate to facilitate private sector development and economic diversification.

13. The authorities, with support from development partners, intend to strengthen the legal and regulatory framework for the financial sector and deepen financial intermediation over the medium term. These reforms include: strengthening the supervisory role of the CBL; developing the capital market; strengthening the payments and settlement system; establishing credit bureaus; introducing a national identification card system; modernizing commercial courts; and increasing access to financial services, particularly in rural areas.

14. Lesotho remains at moderate risk of debt distress (DSA). After taking into account the new nonconcessional external loan for construction of the Metolong dam, the present value of debt to GDP rises slightly above the key threshold level of 40 percent. Throughout the projection period, however, the debt levels remain at manageable levels as fiscal consolidation takes hold. This underscores the need to restore fiscal sustainability and ensure sound debt management. If the significant amount of remittances to Lesotho, averaging at least 20 percent of GDP over the past several years, are taken into account, the 
debt ratios decline significantly and would remain below the remittance-modified threshold of 36 percent, thereby reducing markedly Lesotho's risk of debt distress.
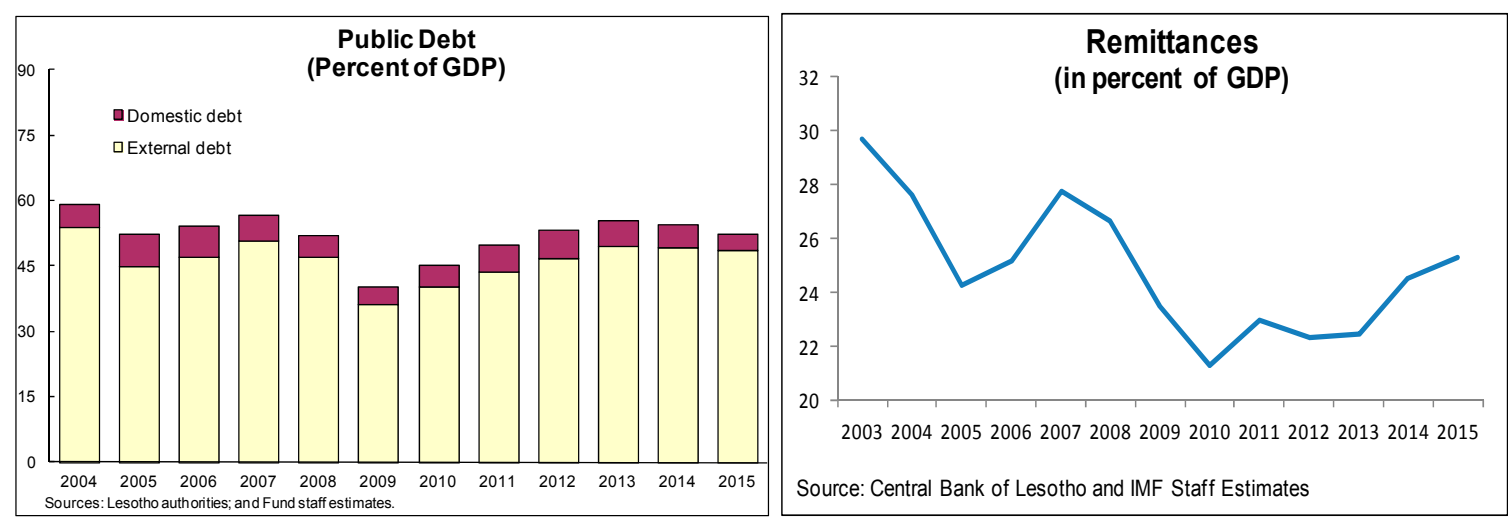

15. In recent years, Lesotho's debt management capacity has improved, supported by technical assistance from some development partners. However, there is scope for further strengthening in light of the increased debt burden and planned issuance of government securities. The authorities plan to strengthen human and institutional capacity in debt management through training and improving the interface between the debt recording system (CS-DRMS) and the IFMIS.

\section{Monetary policy will continue to focus on preserving the peg and maintaining} price stability. Inflation is expected to stabilize at around 5 percent over the medium term, broadly in line with inflation in South Africa. The CBL intends to maintain an adequate level of international reserves to protect the exchange rate peg.

\section{The ECONOMIC Program for 2010/11}

\section{The ECF-supported program would build on the authorities' Interim National} Development Framework, which outlines Lesotho's medium-term development objectives for growth and poverty reduction (MEFP \ 32-35). It would support the authorities' objectives of fiscal consolidation, external stability and broad-based growth for poverty reduction. The ECF would also help strengthen the country's international reserves position thereby enhancing the credibility of the exchange rate peg, bolster confidence in the authorities' policy framework and help to reduce risks.

\section{A. Fiscal Consolidation}

18. Fiscal policy will focus on restraining current expenditures while safeguarding social spending on the poor and vulnerable groups. Against the backdrop of the decline in SACU revenues, fiscal adjustment will rely mainly on recurrent expenditure cuts. 


\section{Box 1. The Metolong Dam and the Lesotho Highlands Water Project}

The construction of the Metolong dam, supported by the MCC and other development partners and the second phase of Lesotho Highlands Water Project (LHWPII) should support growth over the next several years.

The Metolong dam project is part of the authorities' effort to ensure long-term reliable water supply for domestic and industrial purposes. The demand for water in Lesotho has outstripped supply due to rapid urbanization, periodic droughts and increased industrial development. The absence of secure water supply to industries has affected investments, especially in the textile sector.

Construction of the Metolong dam follows feasibility studies and economic assessments supported by the European Development Fund, the Arab Bank for Economic Development in Africa (BADEA), the World Bank and the MCC. These studies identified the Metolong dam program as the least-cost long-term solution for securing medium- to long-term water supply. The MCC estimated an economic rate of return of 24 percent and concluded that the project was viable, and that it would improve industrial production capacity, promote investment in the textile sector, and improve employment opportunities and Lesotho's socio-economic development. Lesotho will also benefit from increased royalties from water transferred to South Africa. The MCC's cost-benefit analysis indicates that the project would not experience cash flow difficulties.

The estimated cost of the project is about US\$413 million and will be financed jointly by Lesotho, the MCC, South Africa, the World Bank, Kuwaiti Fund, OPEC Fund, Saudi Fund and BADEA. Lesotho's share will be met from a non-concessional loan of $€ 140$ million (10 percent of GDP) from the European Investment Bank (EIB) and will be disbursed in rand. The EIB loan is the only one that is non-concessional. Including grants and concessional loans from the MCC, South Africa, the World Bank and the other financiers, the average grant element of the financing package is about 40 percent. The MCC's grant contribution was approved in September 2008 under the MCC's compact program to assist Lesotho reduce poverty through sustained economic growth. The grant has to be utilized by 2013 or Lesotho will lose any remaining balance. Construction of the dam has already started and should be completed by 2013 .

The Lesotho Highlands Water Project (LHWP) is a bi-national project which involves construction of a system of dams and tunnels to transfer water from Lesotho to South Africa, as well as roads and hydroelectricity generation for use in Lesotho. Following the signing of a treaty in 1986 between the two countries, the project has been implemented in phases. The first phase was completed in 2004, and the authorities expect the second phase (LHWP II) to start in 2012 and to be completed by 2017. Financing for LHWPII is currently estimated at M 12 billion (63 percent of 2012/13 GDP), of which Lesotho will be responsible for sourcing M 4 billion and South Africa the rest. A feasibility study of the project is underway. The authorities are aware of the increased risks to debt sustainability arising from this project, and have committed to seek grants and concessional financing for this and other development projects during the program period (MEFP 9 15). Given the uncertainties regarding the financing and timing of the project, staff's analysis, which are reflected in the DSA results, are based on the worst case scenario in which Lesotho is unable to obtain any additional concessional funding. Even under those circumstances, the remittances-modified debt indicators fall below the modified lower thresholds, implying a manageable debt position (DSA). 
The authorities have introduced strong measures to reduce expenditure (as a percent of GDP) on goods and services, subsidies and transfers, ${ }^{4}$ and to limit the increase in the wage bill (Text Table 3; MEFP q 17). The authorities plan to review expenditure with a view to eliminating noncore functions and nonproductive expenditure over the medium term. The World Bank will be undertaking a Public Expenditure Review as part of the Country Assistance Strategy, and the results of the review could be used at a later stage to strengthen safeguarding of key social spending under the program. To mitigate the impact of the fiscal adjustment measures on the vulnerable, the authorities plan to include in the program a floor on

\begin{tabular}{|lc|}
\hline \multicolumn{2}{|c|}{$\begin{array}{c}\text { Text Table 3: Summary of Fiscal Measures 2010/11 } \\
\text { (in percent of GDP) }\end{array}$} \\
\hline Measures & $\begin{array}{c}\text { Expected } \\
\text { Savings }\end{array}$ \\
\hline Revenue Measures & $\mathbf{0 . 4}$ \\
Increase in administrative fees and & \\
charges & 0.3 \\
Increase in fines & 0.1 \\
Expenditure Measures & $\mathbf{8 . 3}$ \\
Reduction in goods and serices & 1.3 \\
Reduction in transfers and subsidies & 4.1 \\
Postponement of payment to defined & 1.8 \\
contribution pension scheme & 1.1 \\
Containment of the wage bill & \\
\hline Source: MoFDP, and Fund staff estimates \\
\hline
\end{tabular}
social spending for old age pension, school feeding program and HIV/AIDs interventions (including anti-retroviral therapies) (MEFP Table 1).

\section{The program envisages increases in public investment for infrastructure} development in support of the authorities' pro-growth objectives. Capital expenditure is projected to increase from 16.0 percent of GDP in 2009/10 to 23.6 percent of GDP in $2010 / 11$, reflecting a substantial increase in externally funded capital spending (6.7 percent of GDP) linked to the construction of the Metolong dam. Of this increase about 0.7 percent of GDP is in the form of grants from the MCC and another 3.6 percent of GDP is to be funded through a nonconcessional loan from the EIB. The authorities consider this project, together with the LHWPII, critical for addressing the infrastructure gap in electricity and water supplies and in supporting private sector activities in key export sectors. In addition, Lesotho will benefit from increases in fiscal revenue and foreign exchange inflows generated by increased water supply to South Africa.

\section{With non-SACU revenues of about 27 percent of GDP, the scope for significant} increases in tax rates is limited, and the main focus will be on implementing measures to improve tax administration. To this end, the 2010/11 budget includes increases in a number of rates, fines and charges, some of which have not been reviewed in over 20 years.

Measures will also be implemented to improve tax administration and compliance, and with technical assistance from the US Treasury Department, the Lesotho Revenue Authority (LRA) is strengthening collection of tax arrears, and enhancing intelligence and investigation, as well as auditing functions. The planned profiling of large-tax-payers with a

\footnotetext{
${ }^{4}$ This includes cuts in transfers to local government and extra budgetary units and bursary scheme for tertiary education.
} 
view to increase tax compliance, and the implementation of the Integrated Revenue Management System should help improve the efficiency of tax collection (MEFP $q 22$ ).

21. Further strengthening of public expenditure management (PEM) will be critical to enhance the efficiency of public expenditure in light of reduced resources. Significant progress has been made in PEM, and for the first time in several years the 2010/11 budget was formulated on the basis of the expected outturn for 2009/10 rather than on the basis of the previous year's budget. This should improve budget execution and spending efficiency as resources will be allocated to ministries with the capacity to implement programs and projects. It should also narrow the gap between the budget and outturn which is a key benchmark for assessing public financial management (PFM) and unlocking additional budgetary support from development partners.

22. The authorities have made progress in improving PFM, with technical and financial assistance from development partners, including the Fund. In April 2009, the authorities introduced the IFMIS to improve budget formulation, execution and accountability. However, there are deficiencies related to internal controls, risk exposure and reporting functions, which have impaired the functionality of the system. The teething problems of the new IFMIS have also resulted in a build-up of domestic arrears, which the authorities are in the process of quantifying. The program incorporates a provision for domestic arrears clearance of M 200 million (1.3 percent of GDP) in the first year (MEFP q21). A March 2010 FAD technical assistance mission made recommendations for improving the functionality of the IFMIS, and some of these recommendations have been incorporated in the program as structural benchmarks for the first review (MEFP $q$ 20, MEFP Table 2).

23. The reduction in revenue has given greater impetus to improving the efficiency and quality of capital expenditure. The Project Appraisal Committee (PAC), which was resuscitated in 2008, vets new capital projects and makes recommendations for inclusion in the budget those projects that meet the medium-term objectives of broad-based growth, poverty reduction and attainment of the MDGs. However, the PAC does not have oversight over ongoing projects. Accordingly, the program includes a structural benchmark for a comprehensive review of all on-going capital projects with the intention of recommending to Cabinet, by end-September 2010, which projects should be retained or eliminated (MEFP 918 ). This is expected to create room for more productive projects in the 2011/12 budget.

\section{B. Structural Reforms to Increase Productivity and External Competitiveness}

24. Implementation of the structural reforms agenda is critical for improving the business climate and increasing external competitiveness and productivity. Relatively high unit labor costs and poor investment climate, evidenced by the slippage in ranking in the 
Doing Business Indicators, have contributed to loss of global and regional competitiveness. This has resulted in a loss of Lesotho's market share of textile exports to the United States. To reverse this trend, the authorities are implementing reforms aimed at reducing the cost of doing business, enhancing competitiveness and productivity, and adopting new legislation to secure property rights, including the Land Reform Bill which was approved by Parliament in March 2010 and is under discussion by the Senate. These reforms are supported by the MCC through its private sector development program and the World Bank under the private sector competitiveness and diversification program, among other projects. The program includes structural measures to improve the business climate (MEFP $q$ 24-26).
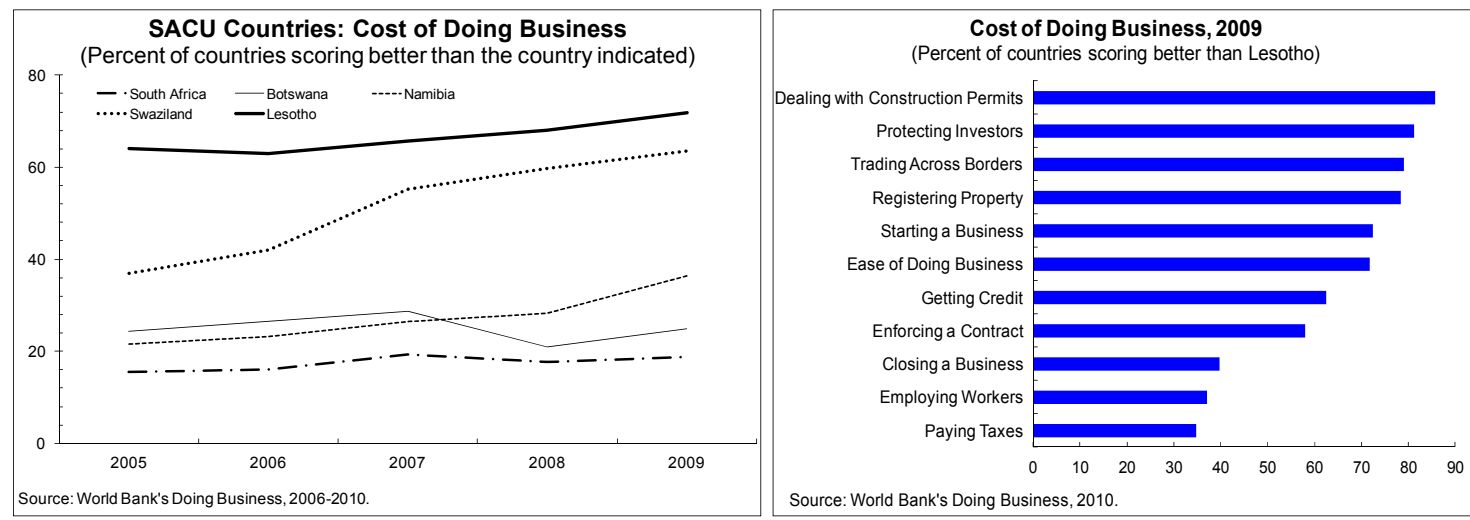

25. A recent staff assessment of the real exchange rate concluded that it is moderately overvalued, and this conclusion remains broadly unchanged. ${ }^{5}$ Given the authorities preference to maintain the peg, there is no scope for external adjustment through a nominal exchange rate depreciation and therefore, restoring current account sustainability will depend on (a) fiscal consolidation; (b) accelerating implementation of structural reforms to increase productivity and reduce unit labor costs; (c) wage restraint to lower cost; and (d) further improvement in infrastructure.

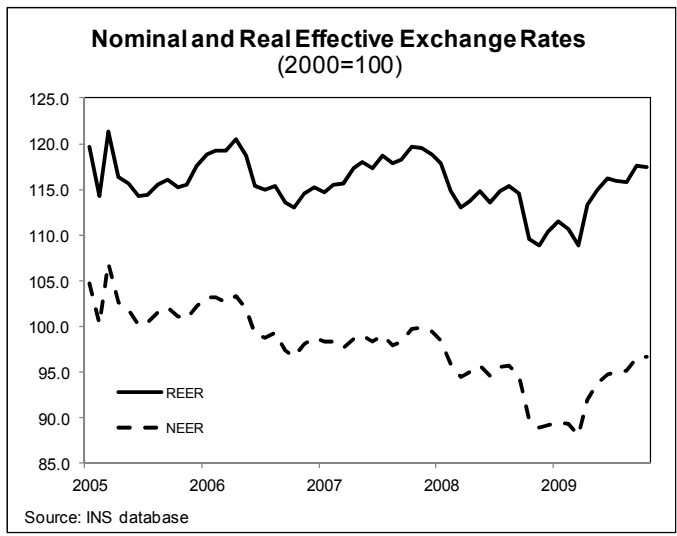

\section{Financial Sector Strengthening to Enhance Access to Financial Services and Support Economic Development}

26. Enhanced prudential regulations of banks and NBFIs will be a main element of financial sector reform under the program (MEFP $\mid \mathbf{2 8 - 2 9}$ ). The CBL intends to submit

\footnotetext{
${ }^{5}$ See Box 1, page 17, SM/10/31, February 9, 2010.
} 
to Parliament, by end-September 2010, the revised Financial Institutions Act (FIA) which incorporates amendments to deal with supervision of NBFIs by the CBL, and unlawful business practices, including Ponzi schemes ${ }^{6}$. Prudential regulations of savings and credit cooperatives (SACCOs) will also be enhanced by amending the Cooperatives Societies Act to prohibit SACCOs from mobilizing deposits from nonmembers. If cooperatives are allowed to take deposits from nonmembers, they will be supervised by the CBL and will be subject to the same prudential and anti-money laundering requirements established for banks.

\section{The program includes reforms to deepen financial intermediation by developing} the domestic bond market and increasing access to financial services, particularly in rural areas (MEFP $\mid \mathbf{2 7}, \mathbf{3 1}$ ). The authorities plan to issue longer-dated domestic bonds. This will extend the yield curve for government securities and provide the authorities with an alternative source of budget financing. In addition, the authorities, with the support of development partners, are reforming the Postbank to shore up its capacity to provide microcredit products in rural areas. The Postbank is also introducing Smartcards which will make it easier for pensioners located in rural areas to access funds.

\section{The CBL is proceeding with the implementation of the Anti-Money Laundering}

(AML) Act of 2008 (MEFP $\$ 30$ ). Draft regulations on the mandate and scope of operations of the Financial Intelligence Unit and for the broader AML regime are being prepared by the CBL and final drafts are expected by end-September 2010.

\footnotetext{
${ }^{6}$ The amendments to the FIA, including on unlawful business practices, were based on recommendations from LEG Department.
} 


\section{Program Risks}

29. Lesotho's economic program is subject to several risks arising from: (a) slower than expected regional and global economic recovery which would further dampen demand for exports and reduce foreign direct investments; (b) further decline in SACU revenues, owing to a change in the revenue-sharing formula and the extent of the economic recovery in South Africa; (c) policy slippages, given the need for expenditure restraint which will require political and social consensus; and (d) delays in implementing key structural reforms underpinning growth in the medium term. One mitigating factor to a number of these risks is the authorities' strong ownership of the program and their commitment to take additional fiscal measures, if warranted, to mitigate risks to the program.

\section{Program Monitoring}

\section{A. Program Monitoring and Conditionality}

\section{Program implementation will be monitored through a set of semi-annual} quantitative performance criteria (PC) and structural benchmarks (SB) laid out in the authorities' MEFP, with terms defined in the accompanying Technical Memorandum of Understanding (TMU). Over the three years of the ECF-supported program (2010-13) semi-annual performance criteria will be set on net domestic borrowing of the central government, and net domestic assets and net international reserves of the CBL. For the first year of the program, performance criteria will be set for end-September 2010 and end-March 2011, with the indicative targets for interim end-quarters June and December 2010. There will also be continuous performance criteria on nonaccumulation of government external payment arrears and on the contracting and guaranteeing of new short-term and medium-term nonconcessional external debt. ${ }^{7}$ Indicative targets will be set on social spending (MEFP 916 ) and on repayment of domestic arrears (MEFP Table 1).

\section{The program will set structural benchmarks in three key reform areas for the} first year of the program: (i) PFM: to enhance the efficiency and quality of public expenditure; (ii) structural reforms: to improve the investment climate, and boost competitiveness and productivity; and (iii) financial sector reforms: to strengthen prudential regulations to reduce systemic risks to the domestic financial sector, and deepen the financial sector by issuing bonds with a view to promote the development of the domestic money and capital market (MEFP Table 2).

\footnotetext{
${ }^{7}$ The concessionality requirements on debt are based on currency denomination rather than residency criterion given the ease with which other Common Monetary Area (CMA) countries, especially South Africa, have unfettered access to debt issued in Lesotho's domestic market. Such access blurs the distinction between external and domestic debt from a residency perspective.
} 


\section{B. Program Financing}

\section{The new ECF arrangement would be part of a wider effort by Lesotho's} development partners (Text Table 4). Total financing requirements for 2010-13 are projected at US\$2,402 million (annual average of 31 percent of GDP). A significant portion of the financing requirement is expected to be covered by SACU transfers, grants and loans from development partners. At the proposed access level for the ECF arrangement, the Fund would account for some 3 percent of envisaged financing by development partners. The financing requirement for 2010 will be financed mainly by SACU transfers, and loans and grants from development partners, including new commitments of US\$83 million for budget support from the African Development Bank (AfDB), EU- including from the Vulnerability Flex, and the World Bank. The first year of the ECF-supported program is fully financed. For the period 2011-13, the commitments from the development partners, including the EU and World Bank, should cover the financing requirements over that period (Table 6).

\begin{tabular}{|c|c|c|c|c|}
\hline \multicolumn{5}{|c|}{$\begin{array}{l}\text { Text Table 4. Lesotho: External Financing Requirements and Sources, 2010-2013 } \\
\qquad \text { (in millions of US dollars) }\end{array}$} \\
\hline & 2010 & 2011 & 2012 & 2013 \\
\hline Gross financing requirements & (540) & (470) & (732) & (660) \\
\hline External current account deficit (excl. official transfers) & (703) & (630) & (704) & (553) \\
\hline Official debt amortization (including IMF) & (28) & (27) & (26) & (29) \\
\hline Other, including change in reserves (+ decrease/- increase) & 190 & 186 & (3) & (78) \\
\hline Sources of financing & 540 & 470 & 732 & 660 \\
\hline IMF & 19.7 & 17.6 & 17.6 & 8.8 \\
\hline Other IFls & 83 & 60 & 46 & 22 \\
\hline AfDB & 9 & 2 & - & - \\
\hline World Bank & 25 & 25 & 25 & 17 \\
\hline EU & 49 & 33 & 21 & 5 \\
\hline Private capital (net) $^{1}$ & (83) & (98) & 29 & 53 \\
\hline Gross official disbursement ${ }^{2}$ & 521 & 490 & 639 & 576 \\
\hline Financing gap (surplus + /deficit -) & - & - & - & - \\
\hline $\begin{array}{l}\text { Sources: Lesotho's authorities; and IMF staff estimates and } \\
{ }^{1} \text { Includes all other net financial flows and errors and omissions } \\
2 \text { Includes loans and grants. }\end{array}$ & & & & \\
\hline
\end{tabular}

\section{Access and Capacity to Repay}

33. The proposed access of 120 percent of quota (SDR 41.88 million), the norm under the ECF instrument, will help build reserves and strengthen the credibility of the loti peg, catalyze budget support from development partners and is consistent with Lesotho's debt sustainability (DSA). ${ }^{8}$ Lesotho's capacity to repay the Fund is adequate, given its low outstanding debt to the Fund (Table 8 ) and previous track record of payment to

\footnotetext{
${ }^{8}$ The IMF financing would be disbursed in seven semi-annual installments starting shortly after the Board approval of the program in May 2010 and following shortly after successful completions of successive reviews (Table 7).
} 
the Fund. ${ }^{9}$ If disbursements are made as scheduled, there will be a peak in debt service to the Fund in 2019 at US\$13.7 million (or 1.1 percent of exports). The strong level of donor support, combined with the prospective economic benefits of future development of new diamond mining and the two large water projects, including water supply to South-Africa (and the accompanying foreign currency earnings and fiscal revenue), are expected to support Lesotho's capacity to repay the Fund.

\section{Safeguards Assessment}

34. The CBL welcomes the IMF's safeguards assessment as it will help strengthen internal control systems. The CBL remains subject to annual external audits. The CBL will work with IMF staff in the coming months to ensure the smooth completion of the safeguards assessment before the time of the first review of the ECF in February 2011. The CBL intends to implement the measures resulting from the safeguards assessment, as part of the effort to strengthen governance and transparency.

\section{E. Data Issues}

35. The data provided are adequate for program monitoring. The quality and timeliness of Lesotho's fiscal, monetary, and external data have improved in recent years, although there are shortcomings. The authorities are committed to building technical capacity within the government and the CBL. The Fund is providing technical assistance in several areas, including public financial management and balance of payment statistics.

\section{Staff APPRAISAL}

36. After several years of good economic performance, Lesotho's fiscal and external balances have deteriorated as a result of the global economic downturn and a surge in spending. The global economic crisis adversely impacted demand for Lesotho's main exports, while the economic downturn in South Africa reduced workers' remittances which further dampened domestic demand. More importantly, the slowdown in imports by South Africa has led to a sharp drop in SACU revenues, at a time when expenditures had reached unprecedented levels, leading to unsustainable fiscal and external positions.

\section{The authorities have designed a macroeconomic and structural reforms} program that, if effectively implemented, will help Lesotho move toward restoring fiscal, debt and external sustainability. The staff supports these policies which seek to address the emerging large fiscal and external imbalances, as a precondition for increasing productivity and competitiveness to diversify the economy and enable broad-based growth

\footnotetext{
${ }^{9}$ Outstanding IMF loans amounted to 38 percent of quota as of March 31, 2010.
} 
for sustained poverty reduction. The proposed measures for strengthening the financial sector and deepening access to financial services in the rural areas should support socio-economic development and help Lesotho move toward achieving the MDGs. The staff welcomes the plan to set up a task team to monitor implementation of the program.

38. Fiscal consolidation is at the center of the authorities' program for restoring macroeconomic stability. In addressing the fiscal imbalance, the authorities have rightly focused on: (a) restraining the growth of recurrent expenditure, especially wages and goods and services which have risen sharply in recent years; (b) prioritizing capital spending by strengthening the role of the Project Appraisal Committee in selecting projects to be included in the budget. The proposed review of on-going capital projects should help strengthen the efficiency of development projects; (c) improving public expenditure and financial management in order to improve the effectiveness of government spending; and (d) strengthening non-SACU revenues mainly by improving tax administration and compliance.

39. The staff welcomes the measures taken in the $2010 / 11$ budget to reduce expenditure and views this as a sign of the authorities' commitment to address the fiscal imbalance. The staff commends the government's intentions to ensure key social programs and spending on the vulnerable are protected, and welcomes the proposed floor on central government social expenditures.

40. The staff notes the increase in the debt burden arising from the loans related to the construction of the Metolong dam and the LHWPII. For Lesotho to maintain debt sustainability, it will have to undertake the necessary fiscal adjustment and implement key structural reforms proposed in the program in order to boost productivity and economic growth. The staff notes the nonconcessional loan for the Metolong dam, and welcomes that grants from other sources, especially the MCC, makes the overall financing package concessional. For the LHWP II and other development projects, the staff urges the authorities to seek grants and concessional financing, including from the World Bank, during the program period.

41. Further strengthening of debt management capacity to effectively manage risks is needed. Although the debt burden remains moderate, there has been a significant increase, and Lesotho will have to strengthen its debt management capacity to ensure long-term debt sustainability. Greater coordination with project implementing agencies will also be critical.

\section{Accelerating structural reforms remains crucial for raising Lesotho's growth} potential by improving the business climate and enhancing external competitiveness and productivity. Lesotho would then be better positioned to exploit its proximity to the larger market of South Africa and the region in general, as a way to diversify its exports and 
develop its manufacturing industry. Effective implementation of structural reforms to support growth will be key to reinforce fiscal consolidation.

43. Strengthening the institutional and regulatory framework for banks and NBFIs should help to support financial sector health and stability. The issuance of domestic bonds to develop the money and capital market is also a welcome development. This, along with efforts to enhance financial services to the rural areas should help to deepen financial intermediation in support of private sector development.

44. The authorities' program is rightly ambitious and necessary, given the extent of the reduction in SACU revenues and the need to return to a path of fiscal, debt and external sustainability. There are risks to the program but without a Fund-supported program, these risks would be amplified. Therefore, the staff supports Lesotho's request for a three-year arrangement under the ECF with access of 120 percent of quota. The arrangement would support the authorities' efforts toward fiscal consolidation while protecting spending on the vulnerable, promote broad-based economic growth for increased employment and poverty reduction, in line with Lesotho's poverty reduction and growth strategy. 
Table 1. Lesotho: Selected Economic and Financial Indicators, 2006-2015 1/

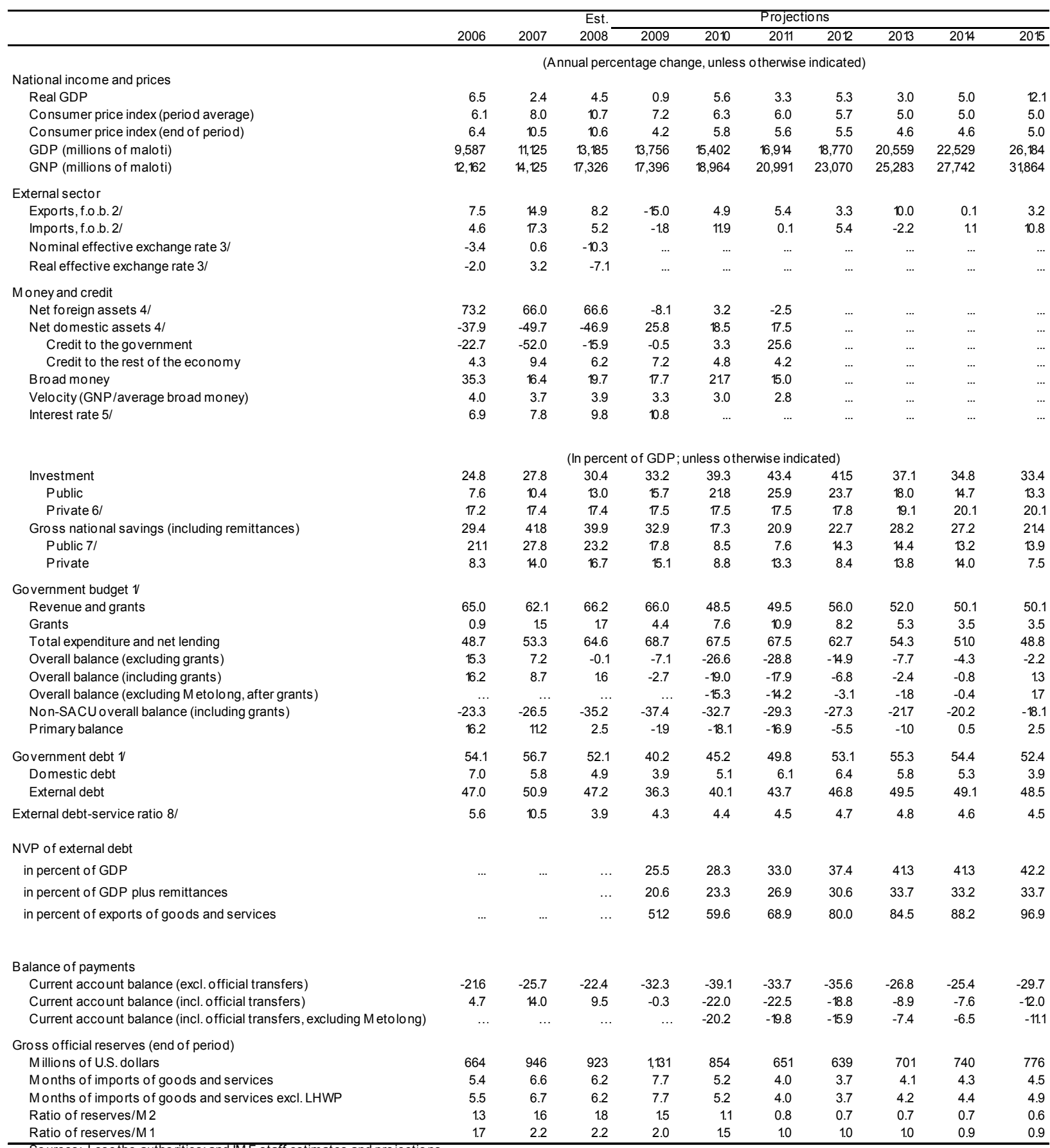

Sources: Lesotho authorities; and IMF staff estimates and projections.

$\checkmark$ Fiscal data are reported on a fiscal year basis; the fiscal year begins in April.

2/ U.S. dollars.

3 / Based on partner-country data, new trade weights from 2004; a minus sign indicates a depreciation.

4/ Change in percent of broad money at the beginning of the period.

$5 /$ The average effective rate on three-month treasury bills.

6/ Includes changes in inventories and gross fixed capital formation by the Lesotho Highlands Development Authority (LHDA).

7/ For 2008-10, government current expenditure has been reduced by the actual and pro spective transfer to the pension fund.

8/ Percent of exports of goods and nonfactor services. 
Table 2. Lesotho: Central Government Operations, 2007/08-2015/16 1/ (In millions of maloti)

\begin{tabular}{|c|c|c|c|c|c|c|c|c|c|c|}
\hline & Actual & Actual & Est. & Budget & Proj. & & & Projections & & \\
\hline & $2007 / 08$ & $2008 / 09$ & $2009 / 10$ & $2010 / 11$ & $2010 / 11$ & $2011 / 12$ & $2012 / 13$ & $2013 / 14$ & $2014 / 15$ & $2015 / 16$ \\
\hline Revenue and grants & 7,225 & 8818 & 9346 & 7854 & 7,654 & 8,605 & 10,754 & 10,936 & 11,752 & 13,382 \\
\hline Revenue & 7,047 & 8,597 & 8,720 & 6,474 & 6,458 & 6,717 & 9,185 & 9,815 & 10,931 & 12,448 \\
\hline Tax revenue & 6,331 & 7,684 & 7,936 & 5,611 & 5,549 & 5,715 & 8,078 & 8,602 & 9,580 & 10,910 \\
\hline Customs revenue (SACU) & 4,098 & 4,901 & 4,918 & 2,162 & 2,162 & 1,975 & 3,947 & 4,081 & 4,544 & 5,174 \\
\hline Noncustoms tax revenue & 2,233 & 2,783 & 3,018 & 3,449 & 3,387 & 3,740 & 4,131 & 4,521 & 5,036 & 5,737 \\
\hline Income taxes & 1,221 & 1,539 & 1,806 & 2,073 & 2,012 & 2,213 & 2,443 & 2,677 & 2,981 & 3,393 \\
\hline Sales tax / value-added tax (VAT) & 848 & 988 & 1,033 & 1,053 & 1,171 & 1,285 & 1,415 & 1,529 & 1,709 & 1,960 \\
\hline Petrol levy & 118 & 99 & 102 & 209 & 108 & 141 & 155 & 170 & 190 & 216 \\
\hline Other tax revenues & 43 & 158 & 72 & 104 & 84 & 95 & 110 & 128 & 137 & 144 \\
\hline Nontax revenue & 716 & 913 & 784 & 864 & 909 & 1,001 & 1,107 & 1,213 & 1,351 & 1,538 \\
\hline Water royalties & 292 & 324 & 347 & 388 & 386 & 425 & 470 & 515 & 573 & 653 \\
\hline Interest received & 7 & 10 & 7 & 8 & 8 & 9 & 10 & 11 & 12 & 14 \\
\hline Other nontax revenues & 371 & 579 & 430 & 468 & 515 & 567 & 627 & 687 & 765 & 871 \\
\hline Grants & 178 & 221 & 626 & 1,379 & 1,195 & 1,889 & 1,569 & 1,121 & 820 & 934 \\
\hline Project grant & & & 212 & 689 & 620 & 683 & 750 & 737 & 820 & 934 \\
\hline$o / w \quad M C C$ & $\ldots$ & $\ldots$ & 414 & 690 & 575 & 1,206 & 819 & 385 & $\ldots$ & $\ldots$ \\
\hline Total expenditure and net lending & 6,210 & 8,605 & 9,730 & 10,927 & 10,651 & 11,723 & 12,054 & 11,433 & 11,946 & 13,029 \\
\hline Current expenditure & 4,687 & 6,385 & 7,266 & 6,923 & 6,932 & 7,135 & 7,679 & 8,049 & 8,787 & 9,590 \\
\hline Wages and salaries & 1,632 & 2,015 & 2,563 & 2,686 & 2,686 & 2,632 & 2,632 & 2,883 & 3,211 & 3,655 \\
\hline Interest payments & 293 & 118 & 117 & 179 & 147 & 187 & 251 & 291 & 300 & 308 \\
\hline External & 243 & 65 & 62 & 114 & 83 & 125 & 169 & 196 & 204 & 212 \\
\hline Domestic & 50 & 53 & 55 & 64 & 65 & 63 & 83 & 96 & 96 & 96 \\
\hline Other expenditure & 2,762 & 4,252 & 4,586 & 4,059 & 4,099 & 4,316 & 4,796 & 4,875 & 5,277 & 5,626 \\
\hline Goods and services & 1,399 & 1,826 & 1,778 & 1,767 & 1,767 & 1,849 & 1,942 & 2,085 & 2,275 & 2,378 \\
\hline Transfer, subsidies and pensions & 1,363 & 2,426 & 2,677 & 2,119 & 2,119 & 2,254 & 2,659 & 2,596 & 2,797 & 3,044 \\
\hline Pensions and gratuities & 396 & 1,013 & 800 & 682 & 682 & 751 & 1,080 & 909 & 1,013 & 1,153 \\
\hline Subventions and transfers & 967 & 1,413 & 1,877 & 1,437 & 1,437 & 1,503 & 1,579 & 1,687 & 1,784 & 1,991 \\
\hline Other Expenditure & $\ldots$ & $\ldots$ & 131 & 173 & 214 & 214 & 194 & 194 & 204 & 204 \\
\hline Capital expenditure & 1,291 & 1,834 & 2,265 & 4,004 & 3,726 & 4,593 & 4,380 & 3,389 & 3,164 & 3,445 \\
\hline Domestically funded & 905.0 & 1,204 & 1,379 & 1,689 & 1,689 & 1,773 & 1,822 & 1,897 & 1,954 & 2,113 \\
\hline Externally funded & 386 & 631 & 885 & 2,315 & 2,037 & 2,820 & 2,558 & 1,493 & 1,211 & 1,332 \\
\hline Net lending & -13 & -1 & 0 & 0 & -7 & -6 & -5 & -5 & -5 & -5 \\
\hline Unidentified Expenditure & 245 & 387 & 200 & $\ldots$ & $\ldots$ & $\ldots$ & $\ldots$ & $\ldots$ & $\ldots$ & $\ldots$ \\
\hline Overall balance, before grants & 837 & -8 & $-1,010$ & $-4,453$ & $-4,193$ & $-5,006$ & $-2,869$ & $-1,618$ & $-1,014$ & -581 \\
\hline Overall balance, after grants & 1,016 & 213 & -384 & $-3,074$ & $-2,997$ & $-3,117$ & $-1,300$ & -497 & -194 & 353 \\
\hline Overall balance excluding Metolong, after grants & $\ldots$ & $\ldots$ & $\ldots$ & $-2,484.2$ & $-2,408$ & $-2,465$ & -586 & -389 & -86 & 461 \\
\hline Non-SACU balance 2/ & $-3,082$ & $-4,688$ & $-5,301$ & $-5,236$ & $-5,159$ & $-5,092$ & $-5,247$ & $-4,578$ & $-4,738$ & $-4,821$ \\
\hline Domestic balance 3/ & 1,453 & 687 & -62 & $-2,023$ & $-2,080$ & $-2,068$ & -147 & 65 & 395 & 958 \\
\hline Primary balance & 1,308 & 331 & -267 & $-2,895$ & $-2,850$ & $-2,930$ & $-1,049$ & -206 & 106 & 661 \\
\hline Total financing & $-1,016$ & -213 & 180 & 2,606 & 2,997 & 3,117 & 1,300 & 497 & 194 & -353 \\
\hline External financing, net & 22 & 173 & 32 & 832 & 1,320 & 1,179 & 1,160 & 225 & 275 & 239 \\
\hline Loan draw ings & 208 & 410 & 259 & 1,074 & 841 & 931 & 989 & 371 & 390 & 398 \\
\hline New commitments & $\ldots$ & $\ldots$ & $\ldots$ & 572 & 718 & 491 & 420 & 150 & 156 & 156 \\
\hline Amortization & -186 & -237 & -228 & -241 & -239 & -242 & -248 & -297 & -271 & -315 \\
\hline Domestic financing, net & $-1,038$ & -386 & -52 & 1,774 & 1,877 & 1,938 & 140 & 272 & -81 & -592 \\
\hline Bank & -999 & -386 & 63 & 1,774 & 1,627 & 1,688 & -25 & 272 & -81 & -592 \\
\hline Nonbank & -39 & 0 & -115 & $\ldots$ & 250 & 250 & 165 & $\cdots$ & $\ldots$ & $\ldots$ \\
\hline Change in domestic arrears & $\ldots$ & $\ldots$ & 200 & $\ldots$ & -200 & $\ldots$ & $\ldots$ & $\ldots$ & $\ldots$ & $\ldots$ \\
\hline Residual gap & 0 & 0 & 204 & 467 & 0 & 0 & 0 & 0 & 0 & 0 \\
\hline
\end{tabular}


Table 2a. Lesotho: Central Government Operations, 2007/08-2015/16 1/ (in percent of GDP; unless otherwise indicated)

\begin{tabular}{|c|c|c|c|c|c|c|c|c|c|c|}
\hline \multirow[b]{3}{*}{ Revenue and grants } & \multirow{2}{*}{\multicolumn{2}{|c|}{\begin{tabular}{|cc} 
Actual & Actual \\
$2007 / 08$ & $2008 / 09$
\end{tabular}}} & \multirow{2}{*}{$\begin{array}{c}\text { Est. } \\
2009 / 10\end{array}$} & \multirow{2}{*}{\multicolumn{2}{|c|}{$\begin{array}{|cc|}\text { Budget } & \text { Proj. } \\
2010 / 11 & 2010 / 11 \\
\end{array}$}} & \multicolumn{5}{|c|}{ Projections } \\
\hline & & & & & & $2011 / 12$ & $2012 / 13$ & $2013 / 14$ & $2014 / 15$ & $2015 / 16$ \\
\hline & 62.1 & 66.2 & 66.0 & 49.8 & 48.5 & 49.5 & 56.0 & 52.0 & 50.1 & 50.1 \\
\hline Revenue & 60.5 & 64.5 & 61.6 & 41.0 & 40.9 & 38.7 & 47.8 & 46.6 & 46.6 & 46.6 \\
\hline Customs revenue (SACU) & 35.2 & 36.8 & 34.7 & 13.7 & 13.7 & 11.4 & 20.5 & 19.4 & 19.4 & 19.4 \\
\hline Noncustoms tax revenue & 19.2 & 20.9 & 21.3 & 21.9 & 21.5 & 21.5 & 21.5 & 21.5 & 21.5 & 21.5 \\
\hline Income taxes & 10.5 & 11.5 & 12.7 & 13.1 & 12.8 & 12.7 & 12.7 & 12.7 & 12.7 & 12.7 \\
\hline Sales tax / value-added tax (VAT) & 7.3 & 7.4 & 7.3 & 6.7 & 7.4 & 7.4 & 7.4 & 7.3 & 7.3 & 7.3 \\
\hline Petrol levy & 1.0 & 0.7 & 0.7 & 1.3 & 0.7 & 0.8 & 0.8 & 0.8 & 0.8 & 0.8 \\
\hline Other tax revenues & 0.4 & 1.2 & 0.5 & 0.7 & 0.5 & 0.5 & 0.6 & 0.6 & 0.6 & 0.5 \\
\hline Nontax revenue & 6.2 & 6.9 & 5.5 & 5.5 & 5.8 & 5.8 & 5.8 & 5.8 & 5.8 & 5.8 \\
\hline Water royalties & 2.5 & 2.4 & 2.4 & 2.5 & 2.4 & 2.4 & 2.4 & 2.4 & 2.4 & 2.4 \\
\hline Interest received & 0.1 & 0.1 & 0.1 & 0.0 & 0.1 & 0.1 & 0.1 & 0.1 & 0.1 & 0.1 \\
\hline Other nontax revenues & 3.2 & 4.3 & 3.0 & 3.0 & 3.3 & 3.3 & 3.3 & 3.3 & 3.3 & 3.3 \\
\hline Grants & 1.5 & 1.7 & 4.4 & 8.7 & 7.6 & 10.9 & 8.2 & 5.3 & 3.5 & 3.5 \\
\hline o/w MCC & $\cdots$ & $\cdots$ & 2.9 & 4.4 & 3.6 & 6.9 & 4.3 & 1.8 & $\cdots$ & $\cdots$ \\
\hline Total expenditure and net lending & 53.3 & 64.6 & 68.7 & 69.2 & 67.5 & 67.5 & 62.7 & 54.3 & 51.0 & 48.8 \\
\hline Current expenditure & 40.3 & 47.9 & 51.3 & 43.9 & 43.9 & 41.1 & 40.0 & 38.2 & 37.5 & 35.9 \\
\hline Wages and salaries & 14.0 & 15.1 & 18.1 & 17.0 & 17.0 & 15.1 & 13.7 & 13.7 & 13.7 & 13.7 \\
\hline Interest payments & 2.5 & 0.9 & 0.8 & 1.1 & 0.9 & 1.1 & 1.3 & 1.4 & 1.3 & 1.2 \\
\hline External & 2.1 & 0.5 & 0.4 & 0.7 & 0.5 & 0.7 & 0.9 & 0.9 & 0.9 & 0.8 \\
\hline Domestic & 0.4 & 0.4 & 0.4 & 0.4 & 0.4 & 0.4 & 0.4 & 0.5 & 0.4 & 0.4 \\
\hline Other expenditure & 23.7 & 31.9 & 32.4 & 25.7 & 26.0 & 24.8 & 25.0 & 23.2 & 22.5 & 21.1 \\
\hline Goods and services & 12.0 & 13.7 & 12.5 & 11.2 & 11.2 & 10.6 & 10.1 & 9.9 & 9.7 & 8.9 \\
\hline Transfers and subsidies & 11.7 & 18.2 & 18.9 & 13.4 & 13.4 & 13.0 & 13.8 & 12.3 & 11.9 & 11.4 \\
\hline Pensions and gratuities & 3.4 & 7.6 & 5.6 & 4.3 & 4.3 & 4.3 & 5.6 & 4.3 & 4.3 & 4.3 \\
\hline Subventions and transfers & 8.3 & 10.6 & 13.3 & 9.1 & 9.1 & 8.6 & 8.2 & 8.0 & 7.6 & 7.5 \\
\hline Other expenditure & $\ldots$ & $\ldots$ & 0.9 & 1.1 & 1.4 & 1.2 & 1.0 & 0.9 & 0.9 & 0.8 \\
\hline Capital expenditure & 11.1 & 13.8 & 16.0 & 25.4 & 23.6 & 26.4 & 22.8 & 16.1 & 13.5 & 12.9 \\
\hline Domestically funded & 7.8 & 9.0 & 9.7 & 10.7 & 10.7 & 10.2 & 9.5 & 9.0 & 8.3 & 7.9 \\
\hline Externally funded & 3.3 & 4.7 & 6.2 & 14.7 & 12.9 & 16.2 & 13.3 & 7.1 & 5.2 & 5.0 \\
\hline Net lending & -0.1 & 0.0 & 0.0 & 0.0 & 0.0 & 0.0 & 0.0 & 0.0 & 0.0 & 0.0 \\
\hline Unidentified Expenditure & 2.1 & 2.9 & 1.4 & $\cdots$ & $\cdots$ & $\cdots$ & $\cdots$ & $\cdots$ & $\cdots$ & $\ldots$ \\
\hline Overall balance, before grants & 7.2 & -0.1 & -7.1 & -28.2 & -26.6 & -28.8 & -14.9 & -7.7 & -4.3 & -2.2 \\
\hline Overall balance, after grants & 8.7 & 1.6 & -2.7 & -19.5 & -19.0 & -17.9 & -6.8 & -2.4 & -0.8 & 1.3 \\
\hline Overall balance excluding Metolong, after grants & $\cdots$ & $\cdots$ & $\ldots$ & -15.7 & -15.3 & -14.2 & -3.1 & -1.8 & -0.4 & 1.7 \\
\hline Non-SACU balance 2/ & -26.5 & -35.2 & -37.4 & -33.2 & -32.7 & -29.3 & -27.3 & -21.7 & -20.2 & -18.1 \\
\hline Domestic balance 3/ & 12.5 & 5.2 & -0.4 & -12.8 & -13.2 & -11.9 & -0.8 & 0.3 & 1.7 & 3.6 \\
\hline Primary balance & 11.2 & 2.5 & -1.9 & -18.3 & -18.1 & -16.9 & -5.5 & -1.0 & 0.5 & 2.5 \\
\hline Total financing & -8.7 & -1.6 & 2.7 & 19.5 & 19.0 & 17.9 & 6.8 & 2.4 & 0.8 & -1.3 \\
\hline External financing (net) & 0.2 & -1.8 & 0.2 & 5.3 & 8.4 & 6.8 & 6.0 & 1.1 & 1.2 & 0.9 \\
\hline Loan draw ings & 1.8 & 3.1 & 1.8 & 6.8 & 5.3 & 5.4 & 5.1 & 1.8 & 1.7 & 1.5 \\
\hline New commitments & $\ldots$ & $\ldots$ & $\ldots$ & $\ldots$ & 4.6 & 2.8 & 2.2 & 0.7 & 0.7 & 0.6 \\
\hline Amortization & -1.6 & -1.8 & -1.6 & -1.5 & -1.5 & -1.4 & -1.3 & -1.4 & -1.2 & -1.2 \\
\hline Domestic financing (net) & -8.9 & -2.9 & -0.4 & 11.2 & 11.9 & 11.1 & 0.7 & 1.3 & -0.3 & -2.2 \\
\hline Bank & -8.6 & $\ldots$ & 0.4 & 11.2 & 10.3 & 9.7 & -0.1 & 1.3 & -0.3 & -2.2 \\
\hline Nonbank & -0.3 & $\ldots$ & -0.8 & $\ldots$ & 1.6 & 1.4 & 0.9 & 0.0 & 0.0 & 0.0 \\
\hline Change in domestic arrears & $\ldots$ & $\ldots$ & 1.4 & $\ldots$ & -1.3 & $\ldots$ & $\ldots$ & $\ldots$ & $\ldots$ & $\ldots$ \\
\hline $\begin{array}{l}\text { Residual gap } \\
\text { Memorandum items: }\end{array}$ & 0.0 & 0.0 & 1.4 & 3.0 & 0.0 & 0.0 & 0.0 & 0.0 & 0.0 & 0.0 \\
\hline GNP at current prices (Millions of maloti) 4/ & 14,125 & 16,538 & 17,396 & 18,964 & 18,964 & 20,991 & 25,283 & 27,742 & 31,864 & 36,279 \\
\hline GDP at current prices (Millions of maloti) 4/ & 11,640 & 13,328 & 14,167 & 15,780 & 15,780 & 17,378 & 19,217 & 21,051 & 23,442 & 26,690 \\
\hline
\end{tabular}

Sources: M inistry of Finance and Development Plannning, and Fund staff estimates and projections.

$\checkmark$ The fiscal year runs from A pril $1 \mathrm{M}$ arch 31.

2/ Overall balance excluding custo ms revenue (SACU)

3/ Domestic balance excludes grants, foreign-financed capital spending, foreign interest payments, and exceptional factors.

4/ On a fiscal year basis. 
Table 3. Lesotho: Balance of Payments, 2006-2015

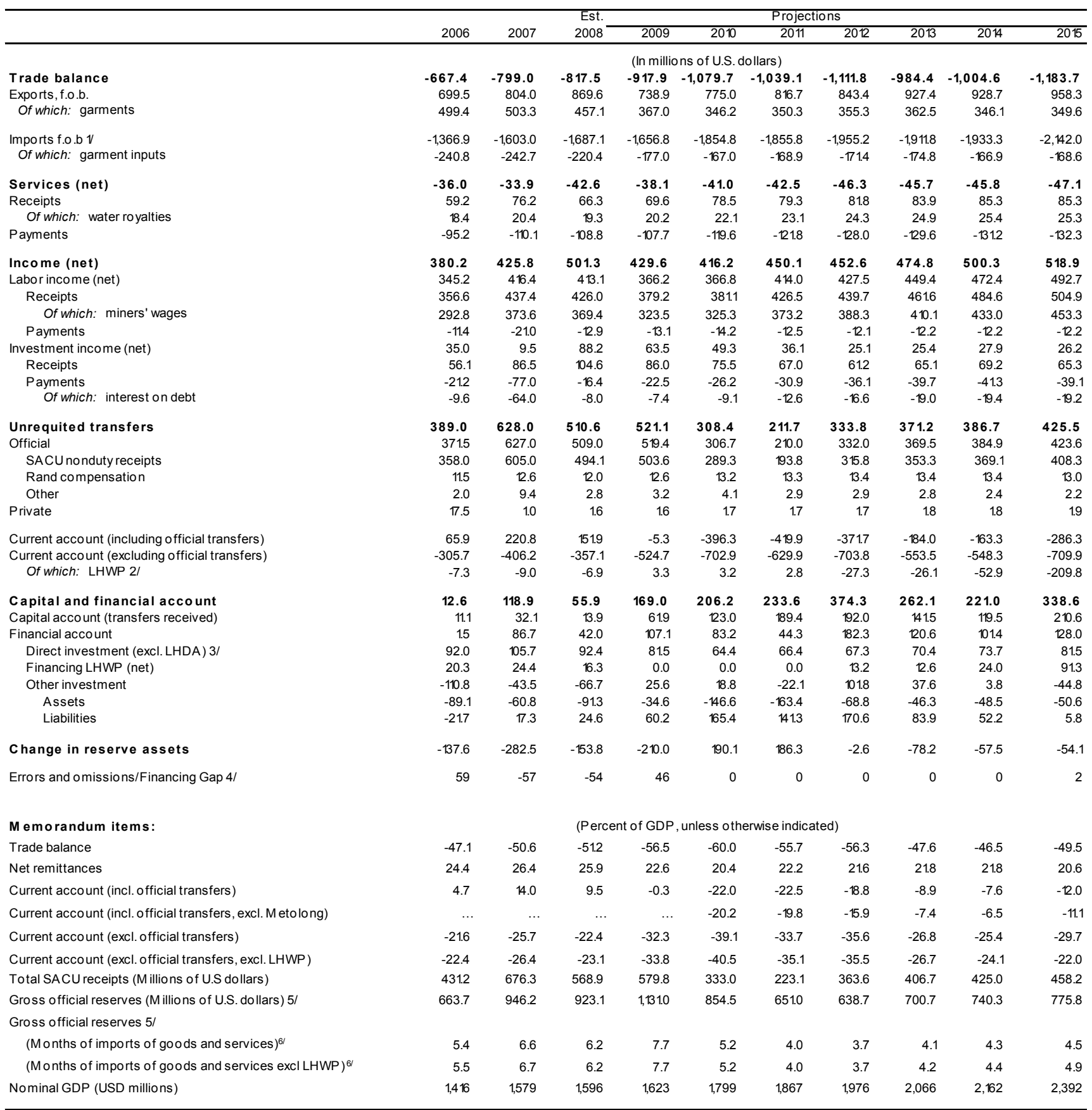

Sources: Central Bank of Lesotho; and IMF staff estimates and projections.

$\checkmark$ Adjusted for SACU duty receipts.

2/ Lesotho Highlands Water Project.

3/ Lesotho Highlands Development Authority.

4/ Fiinancing Gap refers to the period 2010-2015

5/ The figures for gross official reserves include Lesotho's SDR allocation of SDR 29.1mn, received August 28, 2009.

6/ Based on current year imports of goods and services 
Table 4. Lesotho: Monetary Survey, 2006-11

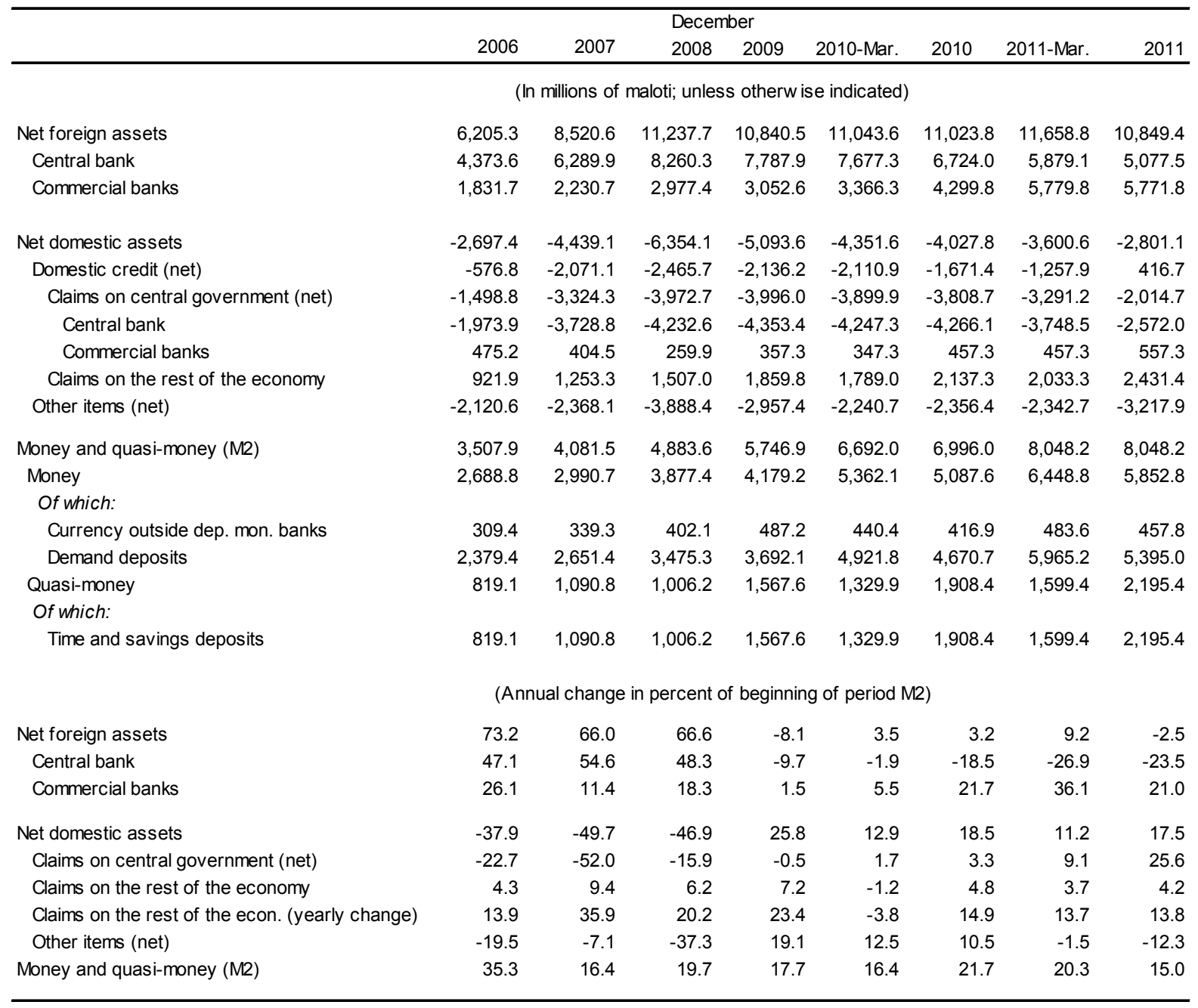

Sources: Central Bank of Lesotho; and IMF staff estimates and projections. 
Table 5: Lesotho: Commercial Banks' Performance Ratios, 2005-09

(As of end-December; in percent)

\begin{tabular}{|c|c|c|c|c|c|}
\hline & 2005 & 2006 & 2007 & 2008 & $\begin{array}{l}\text { Sept. } \\
2009\end{array}$ \\
\hline \multicolumn{6}{|l|}{ I. Capital adequacy } \\
\hline a) Basel capital ratio & 22 & 19 & 14 & 12 & 15 \\
\hline b) Nonperforming loans net & & & & & \\
\hline of provisions to capital & 0.3 & -0.1 & 0.2 & 3 & 2.6 \\
\hline c) Top 20 exposures to statutory & & & & & \\
\hline capital and reserves & 220 & 406 & 520 & 315 & 372 \\
\hline \multicolumn{6}{|l|}{ II. Asset quality } \\
\hline a) Loans to deposit ratio & 29 & 26 & 29 & 30 & 29 \\
\hline b) Earning assets to total assets & 93 & 95 & 96 & 94 & 96 \\
\hline c) Nonperforming loans to total assets & 2 & 2 & 2 & 4 & 0.7 \\
\hline d) Reserve for losses to total loans & 3 & 3 & 3 & 4 & 4 \\
\hline e) Reserve for losses to nonperforming loans & 167 & 125 & 115 & 118 & 113 \\
\hline \multicolumn{6}{|l|}{ |III. Liquidity } \\
\hline a) Liquidity assets to total deposits & 120 & 100 & 94 & 101 & 109 \\
\hline b) Available reserves to total deposits & 8 & 4 & 3 & 4 & 1.1 \\
\hline c) Liquid assets to total assets & 77 & 78 & 76 & 77 & 79 \\
\hline d) Current assets to current liabilities & 86 & 85 & 82 & 83 & 102 \\
\hline \multicolumn{6}{|l|}{ IV. Profitability } \\
\hline a) Net income after tax to total income & 18 & 22 & 21 & 22 & 23 \\
\hline b) Retained income to total income & 16 & 22 & 16 & 32 & 23 \\
\hline c) Total expenses to total income & 81 & 70 & 71 & 69 & 68 \\
\hline d) Return on assets (ROA) & 2 & 2 & 3 & 3 & 4 \\
\hline e) Return on equity & 15 & 27 & 32 & 37 & 50 \\
\hline \multicolumn{6}{|l|}{ V. Solvency } \\
\hline a) Total debt ratio & 11 & 9 & 8 & 8 & 7 \\
\hline
\end{tabular}

Source: Central Bank of Lesotho. 
Table 6. Lesotho: External Financing Requirements and Sources, 2010-2013 (in millions of US dollars)

\begin{tabular}{|c|c|c|c|c|}
\hline & \multicolumn{4}{|c|}{ Projections } \\
\hline & 2010 & 2011 & 2012 & 2013 \\
\hline Gross financing requirements & (540) & (470) & (732) & (660) \\
\hline $\begin{array}{l}\text { External current account deficit (excl. official transfers) } \\
\text { of which: trade balance on goods and services }\end{array}$ & $\begin{array}{r}(703) \\
(1,080)\end{array}$ & $\begin{array}{r}(630) \\
(1,039)\end{array}$ & $\begin{array}{r}(704) \\
(1,112)\end{array}$ & $\begin{array}{l}(553) \\
(984)\end{array}$ \\
\hline $\begin{array}{l}\text { Official debt amortization } \\
\text { of which: IMF repayments }\end{array}$ & $\begin{array}{r}(28) \\
(7)\end{array}$ & $\begin{array}{r}(27) \\
(5)\end{array}$ & $\begin{array}{r}(26) \\
(3)\end{array}$ & $\begin{array}{r}(29) \\
(2)\end{array}$ \\
\hline Other, including change in reserves (+ decrease/- increase) & 190 & 186 & (3) & (78) \\
\hline Sources of financing & 540 & 470 & 732 & 660 \\
\hline IMF & 19.7 & 17.6 & 17.6 & 8.8 \\
\hline Other IFls & 83 & 60 & 46 & 22 \\
\hline AfDB & 9 & 2 & - & - \\
\hline World Bank & 25 & 25 & 25 & 17 \\
\hline EU & 49 & 33 & 21 & 5 \\
\hline Private capital (net)' & (83) & (98) & 29 & 53 \\
\hline FDI & 64 & 66 & 67 & 70 \\
\hline Gross official disbursement ${ }^{2}$ & 521 & 490 & 639 & 576 \\
\hline Official grants & 430 & 399 & 506 & 493 \\
\hline Balance of payments support & 430 & 399 & 506 & 493 \\
\hline Loan disbursements & 61 & 100 & 103 & 53 \\
\hline Financing gap (surplus +/deficit -) & 0 & 0 & 0 & 0 \\
\hline
\end{tabular}

Sources: Lesotho's authorities; and IMF staff estimates and projections.

${ }^{1}$ Includes all other net financial flows and errors and omissions.

2 Includes loans and grants. 
Table 7. Lesotho: Schedule of ECF Disbursements and Reviews, 2010-13

\begin{tabular}{ccc}
\hline Date & $\begin{array}{l}\text { Disbursements 1/ } \\
\text { (Millions of SDR) }\end{array}$ & \multicolumn{1}{c}{ Conditions } \\
2-Jun-10 & 7.80 & Executive Board approval \\
23-Feb-11 & 5.68 & $\begin{array}{l}\text { Completion of first review, based on observance of } \\
\text { performance criteria through September 30, 2010 }\end{array}$ \\
17-Aug-11 & 5.68 & $\begin{array}{l}\text { Completion of second review, based on } \\
\text { observance of performance criteria through } \\
\text { March 31, 2011 }\end{array}$ \\
24-Feb-12 & 5.68 & $\begin{array}{l}\text { Completion of third review, based on observance } \\
\text { of performance criteria through September } \\
\text { 30, 2011 }\end{array}$ \\
20-Aug-12 & 5.68 & $\begin{array}{l}\text { Completion of fourth review, based on observance } \\
\text { of performance criteria through March 31, 2012 }\end{array}$ \\
& 5.68 & $\begin{array}{l}\text { Completion of fifth review, based on observance of } \\
\text { performance criteria through September 30, 2012 }\end{array}$ \\
1-Jun-13 & 5.68 & $\begin{array}{l}\text { Completion of sixth review, based on observance } \\
\text { of performance criteria through March 31, 2013 }\end{array}$ \\
\hline
\end{tabular}

Source: IMF Staff estimates.

1/ Based on access of 120 percent of quota (SDR 41.88 million), disbursements are expected to take place shortly after the date of the Board meeting. 
Table 8. Lesotho: Indicators of Capacity to Repay the Fund ${ }^{1}$

\begin{tabular}{|c|c|c|c|c|c|c|c|c|c|c|c|}
\hline & \multicolumn{11}{|c|}{ Projection } \\
\hline & 2010 & 2011 & 2012 & 2013 & 2014 & 2015 & 2016 & 2017 & 2018 & 2019 & 2020 \\
\hline \multicolumn{12}{|l|}{$\begin{array}{l}\text { Fund obligations based on existing credit } \\
\text { (in millions of SDRs) }\end{array}$} \\
\hline Principal & 4.9 & 4.6 & 3.2 & 1.8 & 1.1 & 0.0 & 0.0 & 0.0 & 0.0 & 0.0 & 0.0 \\
\hline Charges and interest ${ }^{2}$ & 0.0 & 0.0 & 0.0 & 0.0 & 0.0 & 0.0 & 0.0 & 0.0 & 0.0 & 0.0 & 0.0 \\
\hline \multicolumn{12}{|c|}{$\begin{array}{l}\text { Fund obligations based on existing and prospective credit } \\
\text { (in millions of SDRs) }\end{array}$} \\
\hline Principal & 4.9 & 4.6 & 3.2 & 1.8 & 1.1 & 0.8 & 3.3 & 5.5 & 7.8 & 8.4 & 7.6 \\
\hline Charges and interest ${ }^{2}$ & 0.0 & 0.0 & 0.2 & 0.3 & 0.3 & 0.3 & 0.3 & 0.3 & 0.2 & 0.2 & 0.1 \\
\hline \multicolumn{12}{|l|}{ Total obligations based on existing and prospective credit } \\
\hline In millions of SDRs & 4.9 & 4.6 & 3.4 & 2.1 & 1.4 & 1.1 & 3.6 & 5.8 & 8.0 & 8.6 & 7.7 \\
\hline In millions of US\$ & 7.9 & 7.3 & 5.4 & 3.3 & 2.2 & 1.8 & 5.7 & 9.3 & 12.9 & 13.7 & 12.4 \\
\hline In percent of exports of goods and services & 0.9 & 0.8 & 0.6 & 0.3 & 0.2 & 0.2 & 0.5 & 0.8 & 1.1 & 1.1 & 1.0 \\
\hline In percent of debt service ${ }^{3}$ & 23.8 & 20.6 & 15.5 & 9.1 & 5.8 & 4.2 & 15.1 & 22.3 & 28.3 & 27.0 & 22.0 \\
\hline In percent of GDP & 0.5 & 0.4 & 0.3 & 0.2 & 0.1 & 0.1 & 0.2 & 0.4 & 0.5 & 0.5 & 0.5 \\
\hline In percent of Gross International Reserves & 0.9 & 1.1 & 0.9 & 0.5 & 0.3 & 0.2 & 0.5 & 0.9 & 1.2 & 1.3 & 1.2 \\
\hline In percent of quota & 14.0 & 13.0 & 9.7 & 5.9 & 3.9 & 3.2 & 10.2 & 16.6 & 23.0 & 24.5 & 22.1 \\
\hline \multicolumn{12}{|l|}{ Outstanding Fund credit } \\
\hline In millions of SDRs & 24.0 & 30.8 & 39.0 & 42.9 & 41.9 & 41.1 & 37.8 & 32.3 & 24.5 & 16.1 & 8.5 \\
\hline In millions of US\$ & 38.4 & 49.4 & 62.6 & 68.9 & 67.2 & 66.0 & 60.7 & 51.9 & 39.3 & 25.9 & 13.7 \\
\hline In percent of exports of goods and services & 4.5 & 5.5 & 6.8 & 6.8 & 6.6 & 6.3 & 5.6 & 4.6 & 3.4 & 2.1 & 1.1 \\
\hline In percent of debt service ${ }^{3}$ & 116.4 & 139.0 & 178.4 & 189.9 & 175.8 & 156.9 & 159.9 & 124.1 & 86.3 & 51.0 & 24.4 \\
\hline In percent of GDP & 2.2 & 2.7 & 3.2 & 3.4 & 3.2 & 2.8 & 2.5 & 2.1 & 1.5 & 1.0 & 0.5 \\
\hline In percent of Gross International Reserves & 4.5 & 7.6 & 9.8 & 9.8 & 9.1 & 8.5 & 5.5 & 4.8 & 3.7 & 2.4 & 1.3 \\
\hline In percent of quota & 68.7 & 88.2 & 111.7 & 123.0 & 120.0 & 117.8 & 108.4 & 92.6 & 70.2 & 46.2 & 24.4 \\
\hline Net use of Fund credit (in millions of SDRs) & 8.6 & 6.8 & 8.2 & 3.9 & -1.1 & -0.8 & -3.3 & -5.5 & -7.8 & -8.4 & -7.6 \\
\hline Disbursements & 13.5 & 11.4 & 11.4 & 5.7 & 0.0 & 0.0 & 0.0 & 0.0 & 0.0 & 0.0 & 0.0 \\
\hline Repayments & 4.9 & 4.6 & 3.2 & 1.8 & 1.1 & 0.8 & 3.3 & 5.5 & 7.8 & 8.4 & 7.6 \\
\hline \multicolumn{12}{|l|}{ Memorandum items: } \\
\hline Exports of goods and services (in millions of US $\$$ ) & 853.5 & 896.0 & 925.1 & $1,011.3$ & $1,014.0$ & $1,043.6$ & $1,077.4$ & $1,116.7$ & $1,157.8$ & $1,230.9$ & $1,276.8$ \\
\hline Debt service (in millions of US $\$)^{3}$ & 33 & 36 & 35 & 36 & 38 & 42 & 38 & 42 & 46 & 51 & 56 \\
\hline Nominal GDP (in millions of US\$) & 1,744 & 1,800 & 1,941 & 1,998 & 2,113 & 2,318 & 2,390 & 2,464 & 2,540 & 2,618 & 2,699 \\
\hline Gross International Reserves (in millions of US $\$$ ) & 854 & 651 & 639 & 701 & 740 & 776 & 1,105 & 1,089 & 1,065 & 1,073 & 1,064 \\
\hline Quota (millions of SDRs) & 34.9 & 34.9 & 34.9 & 34.9 & 34.9 & 34.9 & 34.9 & 34.9 & 34.9 & 34.9 & 34.9 \\
\hline
\end{tabular}

Source: IMF staff estimates and projections.

${ }^{1}$ ECF disbursements of SDR 7.80 million (18.6 percent of quota) upon approval, June 2010; six sucessive disbursements of SDR 5.68 million (13.6 percent of quota) upon completion of each of the six reviews, through August 2013; in total SDR 41.88 million (120 percent of quota) during 2010-13.

${ }^{2}$ Reflects exceptional relief of all interest payments on concessional loans due to the IMF through December 2011.

${ }^{3}$ Total debt service includes IMF repayments. 
Table 9. Lesotho: Indicators of External Vulnerability, 2006-10

(In percent of GDP; unless otherwise indicated)

\begin{tabular}{|c|c|c|c|c|c|}
\hline & 2006 & 2007 & 2008 & 2009 & $\begin{array}{l}\text { Proj. } \\
2010 \\
\end{array}$ \\
\hline \multicolumn{6}{|l|}{ Financial indicators } \\
\hline Public sector debt ${ }^{1}$ & 54.1 & 56.7 & 52.1 & 40.2 & 45.2 \\
\hline Broad money (M2; annual percent change) & 35.3 & 16.4 & 19.7 & 7.7 & 21.7 \\
\hline Private sector credit (annual change in percent ) & B.9 & 35.9 & 20.2 & 23.4 & 14.9 \\
\hline Domestic credit (net) & -6.0 & -18.6 & -18.7 & -15.5 & -10.9 \\
\hline Treasury-bill yield (percent) ${ }^{2}$ & 6.8 & 8.8 & 0.1 & 6.7 & $\ldots$ \\
\hline Treasury-bill yield (real, percent) ${ }^{3}$ & 0.7 & 0.8 & -0.7 & -0.5 & $\ldots$ \\
\hline \multicolumn{6}{|l|}{ External indicators } \\
\hline Exports of goods and services (U.S. dollars, annual percent change) & 7.9 & 16.0 & 6.3 & -13.6 & 5.6 \\
\hline Imports of goods and services (U.S. dollars, annual percent change) & 3.6 & 17.2 & 4.8 & -1.8 & 11.9 \\
\hline Current account balance (including official transfers) & 4.7 & 14.0 & 9.5 & -0.3 & -22.0 \\
\hline Capital and financial account balance & 0.9 & 7.5 & 3.5 & 10.4 & 11.5 \\
\hline Of which: inward foreign direct investment & 6.5 & 6.7 & 5.8 & 5.0 & 3.6 \\
\hline Net foreign assets of the banking sector (millions of U.S. dollars) & 262.8 & 327.6 & 320.0 & 413.6 & 488 \\
\hline Foreign assets of the banking sector (millions of U.S. dollars) & 273.3 & 338.3 & 329.9 & 455.7 & 524.2 \\
\hline Foreign liabilities of the banking sector (millions of U.S. dollars) & 10.5 & 10.8 & 10.0 & 42.1 & 36.1 \\
\hline Gross official reserves (millions of U.S. dollars) & 663.7 & 946.2 & 923.1 & 1131.0 & 854.5 \\
\hline Gross official reserves (months of imports of goods and services) & 5.4 & 6.6 & 6.2 & 7.7 & 5.2 \\
\hline Ratio of reserve money to reserves (percent) & 14.7 & 0.7 & 8.4 & 2.2 & 2.1 \\
\hline Ratio of broad money to reserves (percent) & 75.8 & 63.3 & 56.9 & 68.9 & 88.9 \\
\hline Total external debt ${ }^{4}$ & 47.0 & 50.9 & 47.2 & 36.3 & 40.1 \\
\hline Ratio of total external debt to exports of goods and services (percent) & 88.7 & 94.3 & 714 & 83.6 & 82.1 \\
\hline REER depreciation (-) (end of period, CPI-based) & -2.0 & 3.2 & -7.1 & $\ldots$ & $\ldots$ \\
\hline \multicolumn{6}{|l|}{ Memorandum items: } \\
\hline GDP (millions of U.S. dollars) & $1,415.8$ & $1,579.1$ & $1,596.0$ & $1,623.4$ & $1,799.3$ \\
\hline Nominal exchange rate (maloti per U.S. dollar, period average) & 6.8 & 7.0 & 8.3 & 8.5 & $\ldots$ \\
\hline Nominal exchange rate (maloti per U.S. dollar, end-of-period) & 7.0 & 6.8 & 9.3 & 7.4 & \\
\hline
\end{tabular}

Sources: Lesotho authorities, and IMF staff estimates.

${ }^{1}$ National government debt on a fiscal year basis; the fiscal year begins in April.

${ }^{2}$ End of period.

${ }^{3}$ Backward-looking with actual $\mathrm{CPI}$.

${ }^{4}$ Excludes private debt within the Common Monetary Area. 
Table 10. Lesotho: Millennium Development Goals

\begin{tabular}{|c|c|c|c|c|c|}
\hline & 1990 & 1995 & 2000 & 2005 & 2008 \\
\hline \multicolumn{6}{|l|}{ Goal 1: Eradicate extreme poverty and hunger } \\
\hline Employment to population ratio, $15+$, total $(\%)$ & 48.0 & 46.0 & 57.0 & 52.0 & 54.0 \\
\hline Employment to population ratio, ages $15-24$, total (\%) & 40.0 & 36.0 & 45.0 & 40.0 & 40.0 \\
\hline Income share held by lowest $20 \%$ &.. & 1 &.. & 3 & .. \\
\hline Malnutrition prevalence, weight for age (\% of children under 5 ) &.. & .. & 15 & 17 & .. \\
\hline Poverty headcount ratio at national poverty line (\% of population) &.. & 48 & .. & 43 & .. \\
\hline Vulnerable employment, total (\% of total employment) &.. &.. & .. &.. & .. \\
\hline \multicolumn{6}{|l|}{ Goal 2: Achieve universal primary education } \\
\hline Literacy rate, youth female (\% of females ages $15-24$ ) &.. & .. & 97 & .. & 98 \\
\hline Literacy rate, youth male (\% of males ages $15-24)$ &.. & .. & 85 &.. & 86 \\
\hline Persistence to last grade of primary, total (\% of cohort) & .. & .. & 54 & 62 & 46 \\
\hline Primary completion rate, total ( $\%$ of relevant age group) & 58 & .. & 60 & 62 & 73 \\
\hline Total enrollment, primary (\% net) & .. & .. & 78 & 75 & 73 \\
\hline \multicolumn{6}{|l|}{ Goal 3: Promote gender equality and empower women } \\
\hline Proportion of seats held by women in national parliament (\%) & .. & 5.0 & 4.0 & 6.0 & 25.0 \\
\hline Ratio of female to male primary enrollment & 122.0 & 112.0 & 104.0 & 100.0 & 99.0 \\
\hline Ratio of female to male secondary enrollment & 142.0 & .. & 131.0 & 127.0 & 132.0 \\
\hline Ratio of female to male enrollments in tertiary education & 130.0 &.. & 153.0 & 127.0 & 119.0 \\
\hline Share of women employed in the nonagricultural sector & .. & 51.6 & 51.0 &.. & .. \\
\hline (\% of total nonagricultural employment) & & & .. & .. & .. \\
\hline \multicolumn{6}{|l|}{ Goal 4: Reduce child mortality } \\
\hline Immunization, measles (\% of children ages $12-23$ months) & 80.0 & 83.0 & 74.0 & 85.0 & 85.0 \\
\hline Mortality rate, infant (per 1,000 live births) & 80.0 & 80.0 & 83.0 & 74.0 & 63.0 \\
\hline Mortality rate, under-5 (per 1,000$)$ & 101.0 & 103.0 & 109.0 & 97.0 & 79.0 \\
\hline \multicolumn{6}{|l|}{ Goal 5: Improve maternal health } \\
\hline Adolescent fertility rate (births per 1,000 women ages $15-19$ ) & .. & .. & 92.0 & 82.0 & 72.0 \\
\hline Births attended by skilled health staff ( $\%$ of total) &.. & 50.0 & 60.0 & 55.0 & .. \\
\hline Contraceptive prevalence (\% of women ages $15-49)$ & 23.0 &.. & 30.0 & 37.0 & .. \\
\hline Maternal mortality ratio (modeled estimate, per 100,000 live births) & .. &.. &.. & 960.0 & .. \\
\hline Pregnant women receiving prenatal care (\%) &.. & 88.0 & 85.0 & 90.0 & .. \\
\hline Unmet need for contraception (\% of married women ages 15-49) &.. & .. & .. & 31.0 & .. \\
\hline \multicolumn{6}{|l|}{ Goal 6: Combat HIVIAIDS, malaria, and other diseases } \\
\hline Children with fever receiving antimalarial drugs ( $\%$ of children under age 5 with fever) & .. & .. & .. & .. & .. \\
\hline Condom use, population ages $15-24$, female (\% of females ages $15-24)$ &.. & .. & .. & 26.0 & .. \\
\hline Condom use, population ages $15-24$, male (\% of males ages $15-24)$ &.. & .. & .. & 44.0 & .. \\
\hline Prevalence of $\mathrm{HN}$, female (\% ages $15-24)$ &.. & .. & .. & 14.9 & 14.9 \\
\hline Prevalence of HV, male (\% ages $15-24)$ & .. & .. & .. & 5.9 & 5.9 \\
\hline Prevalence of HN, total (\% of population ages 15-49) & 0.8 & 14.2 & 23.9 & 23.4 & 23.2 \\
\hline Incidence of tuberculosis (per 100,000 people) & 184.0 & 323.0 & 553.0 & 639.0 & 635.0 \\
\hline Tuberculosis cases detected under DOTS (\%) & .. & 57.0 & 68.0 & 79.0 & 69.0 \\
\hline \multicolumn{6}{|l|}{ Goal 7: Ensure environmental sustainability } \\
\hline CO2 emissions (kg per PPP \$ of GDP) &.. & .. & .. & .. & .. \\
\hline CO2 emissions (metric tons per capita) &.. &.. &.. &.. & .. \\
\hline Forest area ( $\%$ of land area) & 0.2 & 0.2 & 0.2 & 0.3 & 0.3 \\
\hline Improved sanitation facilities (\% of population with access) &.. & 33.0 & 34.0 & 36.0 & 36.0 \\
\hline Improved water source (\% of population with access) &.. & 77.0 & 77.0 & 78.0 & 78.0 \\
\hline Marine protected areas, ( $\%$ of surface area) &.. & .. & .. & .. & 0.0 \\
\hline Terrestrial protected areas ( $\%$ of total land area) &.. & .. & .. & .. & 0.0 \\
\hline \multicolumn{6}{|l|}{ Goal 8: Develop a global partnership for development } \\
\hline Net ODA capita (current US\$) & 87.0 & 65.0 & 19.0 & 34.0 & 70.0 \\
\hline Debt service (PPG and IMF only, \% of exports of G\&S, excl. workers' remittances) & 4.0 & 6.0 & 11.0 & 7.0 & 3.0 \\
\hline Internet users (per 100 people) & 0.0 & 0.0 & 0.2 & 2.6 & 3.6 \\
\hline Mobile phone subscribers (per 100 people) & 0.0 & 1.0 & 2.0 & 3.0 & 4.0 \\
\hline Telephone mainlines (per 100 people) & 1.0 & 1.0 & 1.0 & 2.0 & 3.0 \\
\hline \multicolumn{6}{|l|}{ Other } \\
\hline Fertility rate, total (births per woman) & 5.0 & 5.0 & 4.0 & 4.0 & 3.0 \\
\hline GNI per capita, Atlas method (current US\$) & 540 & 710 & 570 & 800 & 1,060 \\
\hline GNI, Atlas method (current US\$) (billions) & 0.9 & 1.2 & 1.1 & 1.6 & 2.2 \\
\hline Gross capital formation (\% of GDP) & 56.2 & 63.4 & 42.1 & 24.7 & 28.2 \\
\hline Life expectancy at birth, total (years) & 59 & 59 & 50 & 45 & 45 \\
\hline Literacy rate, adult total ( $\%$ of people ages 15 and above) &.. &.. & 86 &.. & 90 \\
\hline Population, total (millions) & 1.6 & 2.0 & 1.9 & 2.0 & 2.0 \\
\hline Trade (\% of GDP) & 132.6 & 138.7 & 131.1 & 153.6 & 158.1 \\
\hline
\end{tabular}

Source: World Development Indicators database 


\section{Attachment I. Letter of Intent}

May 17,2010

Mr. Dominique Strauss-Kahn

Managing Director

International Monetary Fund

Washington, D.C. 20431

U.S.A.

Dear Mr. Strauss-Kahn:

Following several years of improving economic performance, Lesotho's economy has been buffeted by the global economic crisis. Economic activity has slowed owing to a significant reduction in our major exports and reduced workers' remittances from South Africa. In addition, a sharp decline in Southern African Customs Unions (SACU) revenues, which constitute a large proportion of government revenue, is expected to lead to sharply wider fiscal and external imbalances. To address these challenges, the Government of Lesotho has adopted a package of economic policies designed to achieve macroeconomic stability consistent with strong growth and poverty reduction and which is in line with our Interim National Development Framework. In undertaking these policies, the government will ensure social support to the most vulnerable is protected.

The attached Memorandum of Economic and Financial Policies (MEFP) sets forth the government's economic objectives and policies for the three-year period (2010-13) and its action plan for the first year of the program. In support of its program, the government hereby requests a three-year arrangement with the Fund under the Extended Credit Facility (ECF) in a total amount of SDR 41.88 million (equivalent to 120 percent of quota), with the first disbursement of SDR 7.78 million following approval of the program.

The Government of Lesotho believes that the policies set forth in the attached MEFP are adequate to achieve the objectives of the program, but it will take any further measures that may become necessary for this purpose including by strengthening governance and transparency and implementing measures resulting from the updated safeguards assessment of the Central Bank of Lesotho (CBL). Lesotho will consult with the International Monetary Fund (IMF)--at its own initiative or whenever the Managing Director of the IMF requests such a consultation--on the adoption of these measures and in advance of any revisions to the policies contained in the MEFP, in accordance with the IMF's policies on such consultations.

The Government will provide the IMF with such information as the IMF may request in connection with the progress made in implementing the economic and financial policies, and achieving the objectives of the program. The Government authorizes the IMF to publish this letter, the attached MEFP and TMU, and the related Staff Report, including placement of these documents on the IMF website.

Yours sincerely, /s/

Hon. Timothy T. Thahane, Minister of Finance and Development Planning 
Attachment II. Memorandum of Economic and Financial Policies 2010-2013

\section{INTRODUCTION AND BACKGROUND}

1. This memorandum sets out Lesotho's economic and financial policies for the medium term and for the period April 2010-March 2011 for which the government is seeking support from the IMF under a three-year Extended Credit Facility (ECF) arrangement. The policies are consistent with Lesotho's Interim National Development Framework (INDF) which outlines the country's medium-term development objectives for growth and poverty reduction. The government is preparing a comprehensive National Development Plan which is expected to be implemented starting in 2012/13.

2. The main objective of the government's program is to restore macro-economic stability while limiting the adverse impact of fiscal consolidation on the poor and vulnerable Basotho in the face of a protracted decline in revenues. Our program is anchored around three pillars: (a) restoring fiscal sustainability; (b) achieving external sustainability and broad-based growth for sustained poverty reduction; and (c) strengthening the financial sector and enhancing access to financial services. Without decisive actions, Lesotho faces severe fiscal and balance of payments pressures that could erode the recent gains the country has made on macroeconomic stabilization.

\section{RECENT ECONOMIC DEVELOPMENTS}

3. After several years of favorable macro-economic management, Lesotho's economic performance deteriorated in 2009 in the wake of the global crisis. Real GDP growth decelerated to 0.9 percent from 4.5 percent in 2008, mainly reflecting a marked decline in textile exports, slow recovery in diamond exports, and a decrease in workers' remittances from South Africa which lowered household disposable income and reduced consumption. This also led to a shift in the current account position from a surplus of 9.5 percent of GDP in 2008 to a deficit of 0.3 percent of GDP in 2009.

4. On the fiscal front, after five consecutive years of surpluses, the fiscal position is expected to shift to a deficit of 2.7 percent of GDP in 2009/10 from a surplus of 1.6 percent in 2008/09. This was on account of increased spending on (a) public sector wages; (b) new hiring in the education and health sectors; (c) social safety net for vulnerable groups; (d) funding of the newly-established defined contribution pension scheme;

(e) developing a Minimum Infrastructure Platform to support private investments; and

(f) strengthening institutions that will support sound economic management.

5. Inflation slowed sharply to 4.2 percent (year-on-year) in December 2009 from 10.6 percent in December 2008, reflecting much smaller increases in food prices and falling international fuel prices. Nevertheless, fertilizer prices and other input costs remain 
high, and this has had a negative impact on agricultural production. The rate of inflation is highly influenced by inflation developments in South Africa.

6. The financial sector has weathered the global crisis well. Our banks are well capitalized, profitable and liquid. The major vulnerability to our financial system stems from weakly supervised nonbank financial institutions (NBFIs) but efforts are underway to strengthen the CBL's supervisory role over NBFIs. Significant progress has been made in anti-money laundering reforms including implementation of "Know Your Customer" guidelines.

\section{Medium-Term ObJectives ANd Policies}

7. Lesotho faces serious macro-economic challenges over the medium-term. The severity of the global economic crisis has resulted in (a) steep declines in SACU revenues which account for the bulk of our revenues; (b) a sharp contraction in demand for our main exports of textile and diamonds; and (c) a reduction in workers' remittances following the retrenchment of Basotho workers in South Africa. Lower imports by South Africa are expected to result in a significant reduction of net SACU revenues by a total of 23.3 percent of GDP during 2010/11-2011/12 relative to 2009/10. Although some recovery in SACU receipts is expected in 2012/13, we anticipate a permanent loss of SACU revenues with receipts stabilizing at around 20 percent of GDP, levels prevailing prior to the change in the SACU formula in 2004/05 and before the economic boom in South Africa.

\section{The decline in SACU revenues comes at a time when expenditures are at} historically high levels, and is projected to widen the fiscal deficit (excluding outlays for the Metolong dam) to an average of 15.0 percent of GDP in 2010/11-2011/12. The government plans to finance the deficit mainly by drawing down its deposits at the CBL and by issuing domestic bonds. In addition, we have secured budgetary grants and concessional loans from some of our development partners.

9. Starting with the $2010 / 11$ budget, the Government has began to take determined action to curtail recurrent spending with a view to reducing nonproductive expenditures over the medium-term to a level consistent with sustainable levels of revenues. In this regard, the government intends to reduce total expenditure from around 68 percent of GDP in 2010/11 to around 49 percent of GDP by 2015/16, by cutting nonpriority expenditures while at the same time protecting spending on critical social programs. The reduction in capital spending in part reflects the completion of the Metolong dam in 2012/13. With medium-term revenues stabilizing at around 50 percent of GDP, we are targeting a balanced fiscal position by 2015/16. 
10. Specific measures to reduce recurrent spending include:

- Containing the wage bill by: (a) restricting new recruitment to high-priority areas; (b) freezing the number of existing positions and reducing the size of the public service; and (c) eliminating posts that have remained vacant for more than 12 months. If there is need for any new positions, they will be assessed in the context of skills requirements in high-priority areas such as health and education. Given the general wage increases in each of the last three years, government will eschew further general wage increases during the program period. Any increases will be linked to performance and retaining key skills. In addition, the government will take advantage of the mandatory retirement scheme for civil servants that comes into effect in 2010/11 to reduce the size of the public service by carefully assessing whether the vacated positions can be eliminated without affecting efficient services delivery. We intend to carry out a comprehensive review of the public service to identify noncore functions of the government with a view to reducing certain nonessential operations.

- Reducing expenditure on goods and services by: (a) rationalizing diplomatic missions; (b) reducing international travel; and (c) cutting spending on furniture and equipment. In addition, we will be outsourcing noncore functions to enhance efficiency gains.

11. By containing the growth in recurrent spending, we will create fiscal space to increase development spending and to raise the share of our spending allocated to capital projects. This will release funds for developing a minimum infrastructure platform and institutions for investment, particularly small and medium scale enterprises, which is a key priority of the government.

12. The external current account deficit (excluding outlays for the Metolong dam) is projected to widen to 20 percent of GDP in 2010 owing to the lower SACU revenues. It should narrow thereafter to about 6.5 percent of GDP by 2014 as net SACU revenues recover and as we implement the fiscal adjustment measures. With the proposed adjustment, our international reserves should be sufficient to sustain the loti peg to the rand.

13. Growth over the medium term is premised on the implementation of our macroeconomic and structural reforms aimed at increasing competitiveness and achieving diversification. Real GDP is expected to accelerate from 0.9 percent in 2009 to an average of 4.3 percent per annum during the ECF-supported program (2010-2013). An expansion in private sector activities, complemented by public investment in infrastructure, including the construction of the Metolong Dam, the start up of the second phase of the 
Lesotho Highlands Water Project (LHWP II) and development of further mining opportunities will help to underpin growth.

14. There is limited scope for independent monetary policy due to our country's membership in the Common Monetary Area (CMA) and the peg of the loti to the South African rand. The peg of the loti to the rand has facilitated transactions with South Africa and helped maintain low inflation. In this context, our efforts will continue to focus on preserving the fixed exchange rate system and maintaining price stability. Supported by the peg, inflation is expected to stabilize at around 5 percent over the medium term, broadly in line with inflation in South Africa. The CBL will maintain an adequate level of international reserves to protect the exchange rate peg.

15. With regards to the proposed loans for the Metolong dam and LHWPII, we are aware that these will raise our debt levels over the medium term. However, these projects are critical to addressing sustained economic growth and poverty reduction. Nevertheless, we are aware of the risks of a rising debt burden and are committed to seeking grants and concessional financing for the LHWPII and other development projects during the program period.

\section{MACROECONOMIC OBJECTIVES FOR 2010/11}

The government's economic program aims to achieve fiscal consolidation, external stability and broad based growth for poverty reduction, and a strengthening of the financial sector. Below we describe the specific policies we intend to put in place to achieve these broad objectives. The specific quantitative targets and structural policies underlying our adjustment program are summarized in Tables 1 and 2.

16. The Government recognizes the urgent need to address emerging large fiscal imbalances given the steep and permanent decline in SACU revenues. This will require a judicious balance between adjustment measures, external support and use of accumulated government deposits. The fiscal savings generated in recent years provide some scope to draw down reserves without jeopardizing the CBL's reserve position and the credibility of the loti peg to the rand. The bulk of the proposed fiscal adjustment will rely mainly on curtailing nonproductive and nonpriority expenditures while safeguarding vulnerable groups. The program includes a floor for spending on priority social programs.

17. In the absence of policy adjustment, the decline in SACU revenues by 21 percent of GDP in 2010/11 would have resulted in a fiscal deficit (excluding outlays for the Metolong dam) of about 20 percent of GDP in 2010/11. However, the government intends to implement bold measures to contain the fiscal deficit (excluding outlays for the Metolong dam) to 15.3 percent of GDP by reducing overall spending (excluding outlays for the 
Metolong dam) by 4.9 percent of GDP. These spending cuts largely fall on recurrent expenditure as follows:

- Limiting the increase in the wage bill to 4.8 percent in nominal terms to cater for inflation and increased hiring in the health and education sectors;

- Reducing goods and services appropriation from 12.5 percent of GDP in 2009/10 to 11.2 percent of GDP, below the level of 2007/08; and

- Limiting spending on subsidies and transfers, mainly through the postponement to 2012 of the payment of M 250 million linked to pension reform, and reducing appropriation for the bursary scheme for tertiary education by M104 million.

\section{With regard to public investment, the government realizes there is scope to} improve the efficiency of capital projects. In this context, we intend to conduct a comprehensive review of all ongoing capital projects with a view to assessing their desirability and making recommendations to Cabinet on which projects should be retained or eliminated. This review, which will also look at recurrent expenditures, (including on the bursary scheme for tertiary education) will be submitted to Cabinet by September 2010 in order to help guide budget preparations for 2011/12. For new capital projects, the Project Appraisal Committee will continue to vet them to ensure that only those that meet the criteria for enhancing broad-based economic growth, poverty reduction, and attaining the MDGs are included in the budget.

19. With reduced revenues, government recognizes the importance of improving public expenditure management (PEM) to enhance efficiency of expenditure. With this in mind, for the first time in several years, the 2010/11 budget was framed based on the expected outturn rather than on the previous year's budget. This is a major improvement over the previous process, and will contribute to improved budget execution as resources are allocated to those ministries that have the capacity to execute their programs.

20. We are also committed to building on the progress we have made in reforming our Public Financial Management (PFM) system. The recent implementation of the Integrated Financial Management Information System (IFMIS) will help improve budget formulation, execution, and reporting. However, we are aware of the significant deficiencies in the functioning of the IFMIS and are committed to make progress in this area guided by the recommendations of the recent IMF Technical Assistance mission on PFM. Improving the functionality of the IFMIS will include actions to prevent over-commitment of resources 
and to strengthen internal control. ${ }^{1}$ These actions will be monitored through structural benchmarks.

21. The government is aware that there has been an accumulation of domestic arrears, mainly because of teething problems with the IFMIS rather than due to lack of funds. We are in the process of clarifying the size of the arrears as at end-March 2010, with a view to eliminating them within the program period. For the first year, 2010/11, we plan to eliminate up to M 200 million. We intend to undertake a survey from line ministries to complement information from the IFMIS in order to get a more accurate figure for domestic arrears. Lesotho has no external payments arrears.

\section{Given that non-SACU revenues are relatively high, at around 27 percent of} GDP, there is limited scope for further increases. However, as announced in the 2010/11 budget, the government will undertake a wholesale revision of rates, charges and fines, some of which have not been reviewed in 20 years. In addition, government believes there is some scope to increase non-SACU revenue by improving tax administration and compliance. To this end, with technical assistance from the United States, the Lesotho Revenue Authority (LRA) has taken steps to strengthen (a) collection of tax arrears; (b) intelligence and investigations; and (c) audit. The LRA is also profiling large tax payers to increase tax compliance. Implementation of the Integrated Revenue Management System, complemented with training of LRA managers and tax collectors, will help improve overall operational efficiency.

\section{The government recognizes the importance of having unqualified audited} financial statements for the purpose of strengthening governance and public accountability. To this end, preparation of the annual public financial accounts for 2007/08 has been completed and the Statement of Affairs as at end-March 2008 is at an advanced stage. Once approved, this will set the stage for preparation of the annual public financial accounts for subsequent years, starting with 2008/09. The legal framework for budget execution is also being revised and the draft Public Financial Management and Accountability Bill will be submitted to Cabinet by June 2010.

\section{Structural Reforms to Improve Productivity and The INVESTMENT Climate}

\section{Improving the investment climate to boost competitiveness and productivity by} lowering unit labor costs is critical for broad-based shared-growth and diversification.

\footnotetext{
${ }^{1}$ The IFMIS allows over commitments when it processes payment vouchers by reversing the related commitment, thereby releasing funds previously committed. In addition, users belonging to one ministry maybe able to access the records of another ministry indicating inadequate access control.
} 
To this end, the government is pursuing reforms that will make it easier to start, operate and expand businesses. A number of these reforms are supported by development partners including the Millennium Challenge Corporation (MCC), which is pursuing reforms in the following areas: (a) modernize civil legal procedures to expedite resolution of commercial disputes; (b) undertake land reform to facilitate use of land as an economic asset;

(c) introduce national identification cards; (d) establish a credit reference bureau; (e) develop an automated payments and settlement system; and (f) reform the Postbank to expand access to financial services in the rural areas.

25. The government is committed to improving the investment climate in order to attract increased private sector investment and create employment opportunities for any retrenchment arising from public sector reform. Effective implementation of the MCC's private sector component and the World Bank's ongoing Private Sector Competitiveness and Diversification Program, among other projects, should help reduce the cost of doing business and boost Lesotho's regional and global competitiveness. To this end, we intend to submit to Parliament both the Companies (a structural benchmark under the program) and the Industrial Licensing Bills by end-September $2010 .^{2}$

26. Since government intends to maintain the loti peg to the rand, achieving external sustainability will rely on fiscal consolidation, fast-tracking of key structural reforms to enhance productivity growth in tradables, wage restraint to reduce costs, and improvement of infrastructure. In light of Lesotho's close proximity to South Africa and comparatively lower unit labor costs, we will intensify our efforts to take advantage of this large market as a way of developing our manufacturing industry.

\section{Strengthening the Financial Sector}

27. Deepening the money and capital markets will be a key priority of the government. The development of a domestic bond market will help extend the yield curve, deepen the securities market, and provide government with an alternative source of financing for priority projects. The government believes there is significant demand from the banking sector and institutional investors for longer-dated investment instruments. The CBL plans to issue bonds by December 2010. This is a structural benchmark under the program.

\section{The CBL is reviewing the Financial Institutions Act (FIA) and also intends to strengthen the framework for prudential regulations of savings and credit cooperatives}

\footnotetext{
${ }^{2}$ The Companies Bill will enhance the ease of starting business by improving procedures and reducing cost. It will also improve on investor protection, specifically minority shareholders. The Industrial Licensing Bill will reduce the cost of doing business by facilitating registration of companies and easing licensing requirements. Both reforms are being supported by the World Bank.
} 
(SACCOs), and other NBFIs including pension funds. The review of the FIA has been mostly completed, and incorporates amendments which deal with the legal framework to underpin the CBL's supervision of NBFIs, and prohibition of unlawful business practices, including Ponzi schemes. The CBL expects to submit to Parliament the amendments to the FIA by end-September 2010. This is a structural benchmark under the program.

29. The legal and supervision status of SACCOs that mobilize deposits from nonmembers is under review by the CBL and the Commissioner of Cooperatives. The intention is to delete, by end-December 2010, Article 68 of the Cooperatives Societies Act which legalizes cooperatives to mobilize deposits from nonmembers. If a decision is taken to allow cooperative banks to take deposits from nonmembers, those institutions would have to be supervised by the central bank and would be subject to the same prudential and antimoney laundering requirements established for regular banks.

30. The Financial Intelligence Unit, which was established in 2008 following parliamentary approval of the Anti-Money Laundering (AML) Act, is currently being set up in consultation with development partners. Draft regulations for the unit's mandate and for the broader AML regime are being prepared by the CBL with technical assistance from the US Treasury Department, and final drafts are expected by end-September 2010.

31. The government, with the support of MCC and the International Fund for Agricultural Development (IFAD) is committed to improving access to financial services for rural areas. In this context, the Postbank has started to provide micro-lending products to individuals and will expand this service to small and medium-size enterprises. It will also introduce Smart-cards which will make it easier for pensioners to receive and withdraw funds. As the size of its micro-lending operations increases, the CBL will closely monitor the Postbank's operations.

\section{Poverty Reduction Strategy}

32. The government's economic program to be supported by the ECF is consistent with the strategy of achieving broad based growth and poverty reduction set out in the Interim National Development Framework (INDF) (2008/09-2010/11). The INDF is based on the priorities and objectives contained in the original Poverty Reduction Strategy Paper (PRSP) (2004/04-07/08). It identifies as a key priority the need to address Lesotho's vulnerability to over-reliance on remittances from South Africa as well the significant dependence of the budget on SACU revenues.

33. To address this vulnerability, the INDF focuses on achieving sustained economic growth as a way to diversify its revenue base and generate alternative employment opportunities for Basotho. The INDF growth strategy points to specific sectors for diversification as manufacturing, mining, tourism and agriculture. Growth would be driven 
by the private sector, while the government's role would be to provide an appropriate environment through (i) sound macro-economic management; (ii) a conducive investment climate; (ii) provision of infrastructure; and (iv) a sound financial system. The INDF also acknowledges the need for reducing government spending to align it with sustainable revenues, and emphasizes the importance of improving the effectiveness and efficiency of the remaining expenditure, while at the same time protecting vulnerable groups such as pensioners and those with HIV/AIDS.

34. The proposed ECF would support these objectives in the INDF as follows: (i) it aims to achieve macro-economic stability through an orderly fiscal adjustment that addresses the reduction in SACU revenues; (ii) it focuses on raising non-SACU revenues and improving public expenditure and financial management; (iii) it calls for accelerating structural reforms in order to increase productivity and competiveness which would facilitate economic diversification; and finally (iv) the program targets to strengthen the financial sector by addressing vulnerabilities from NBFIs and improving access to financial services in rural areas. The ECF-supported program also protects the vulnerable by putting a floor on critical social expenditures, including on HIV/AIDS.

\section{The INDF is a precursor, to a comprehensive five-year National Development} Plan (NDP) which is planned to be implemented starting 2012/13. By mid-2010, we plan to embark on the process of developing the NDP with wide participation of key stakeholders across the country. The NDP will aim at achieving Lesotho's development objectives of strong sustained growth for poverty reduction, as a way towards achieving the MDGs.

\section{Risks and Contingency Plans}

36. Although there are some signs of a regional and global recovery, some downside risks to the program remain. These risks relate to: (i) the pace of the global and regional economic recovery; (ii) potentially lower SACU revenues because of: a change in the SACU revenue-sharing formula; a reduction in tariff rates due to trade liberalization; and the creation of a SADC customs union. If such risks were to materialize, the government, in close consultation with IMF staff, stands ready to take additional fiscal measures while safeguarding social spending to ensure the achievement of a sustainable fiscal position and to prevent a large reduction of reserves.

\section{SAFEguard ASSESSMent}

37. The CBL welcomes the IMF's safeguards assessment and we believe it will help the CBL strengthen its internal control systems. The CBL's financial statements are externally audited by an independent external audit firm. We look forward to the findings and recommendations of the safeguards report and CBL will work with IMF staff in the 
coming months to ensure the smooth completion of the safeguards assessment by the time of the first review of the ECF in February 2011.

\section{Program Monitoring}

38. Semi-annual disbursements under the ECF arrangement will be based on the observance of quantitative performance criteria. Completion of the first and second reviews of the arrangement, by February 23, 2011 and August 17, 2011, respectively, will be based on the observance of quantitative performance criteria through end-September 2010 and end-March 2011, respectively (Table 1). The targets for end-June 2010 and December 2010 will be indicative. The definitions of the variables monitored as quantitative performance criteria are provided in the Technical Memorandum of Understanding (TMU).

39. Government is committed to ensuring that the program remains on track, and has set up a task team chaired by the Ministry of Finance and Development Planning to monitor implementation. 


\section{Table 1. Lesotho: Quantitative Performance Criteria, Benchmarks, and Indicative Targets \\ March 2010-March 2011}

Table 1. Lesotho: Quantitative Performance Criteria, Benchmarks, and Indicative Targets March 2010 - March 2011

\begin{tabular}{|c|c|c|c|c|c|c|}
\hline & \multirow[b]{2}{*}{$\begin{array}{l}\text { December, } 2009 \\
\text { Actual }\end{array}$} & \multicolumn{4}{|c|}{2010} & \multirow{2}{*}{$\begin{array}{c}2011 \\
\text { March } \\
\text { Perf. Criteria }\end{array}$} \\
\hline & & $\begin{array}{l}\text { March } \\
\text { Est. }\end{array}$ & $\begin{array}{c}\text { June } \\
\text { Benchmarks }\end{array}$ & $\begin{array}{l}\text { September } \\
\text { Perf. Criteria }\end{array}$ & $\begin{array}{c}\text { December } \\
\text { Benchmarks }\end{array}$ & \\
\hline & \multicolumn{6}{|c|}{ (In millions of Maloti) } \\
\hline requirement of the central government ${ }^{1,2,3}$ & $\ldots$ & 0.0 & -217.5 & 311.5 & 779.9 & $1,877.0$ \\
\hline \multirow[t]{2}{*}{$\begin{array}{l}\text { Ceiling on the net domestic assets } \\
\text { of the Central Bank of Lesotho }{ }^{1,2,3}\end{array}$} & - & - & 1,170 & 1,884 & 1,752 & 3,032 \\
\hline & \multicolumn{6}{|c|}{ (In millions of US dollars) } \\
\hline $\begin{array}{l}\text { Floor on the stock of net international } \\
\text { reserves of the Central Bank of Lesotho }{ }^{2,3}\end{array}$ & 1,118 & 1,027 & 992 & 956 & 884 & 827 \\
\hline \multicolumn{7}{|l|}{$\begin{array}{l}\text { Ceiling on the amount of new non- } \\
\text { concessional external debt contracted or } \\
\text { guaranteed by the public sector (cumulative } \\
\text { from end-March } 2010^{2,4}\end{array}$} \\
\hline Maturity of less than one year & $\ldots$ & 0 & 0 & 0 & 0 & 0 \\
\hline Maturity of one year or more ${ }^{1,5}$ & $\ldots$ & 0 & 182 & 182 & 182 & 182 \\
\hline $\begin{array}{l}\text { Ceiling on the stock of external payments } \\
\text { arrears }{ }^{4}\end{array}$ & $\ldots$ & 0 & 0 & 0 & 0 & 0 \\
\hline Indicative targets: & \multicolumn{6}{|c|}{ (In millions of Maloti) } \\
\hline Floor on the central government social expenditures ${ }^{6}$ & $\ldots$ & 0 & 170 & 170 & 170 & 170 \\
\hline Ceiling on gross cumulative payments of domestic arrears & & 0 & 200 & 200 & 200 & 200 \\
\hline \multicolumn{7}{|l|}{ Memo items: } \\
\hline Net disbursements ${ }^{1,2}$ & & 0 & 212.2 & 198.9 & 208.0 & 396.3 \\
\hline General budget support & $\ldots$ & 0 & 298.0 & 358.0 & 448.0 & 718.0 \\
\hline Debt service payments & $\ldots$ & $\ldots$ & 85.8 & 159.1 & 240.0 & 321.7 \\
\hline Unused outstanding Metolong balances ${ }^{1}$ & $\ldots$ & $\cdots$ & 1279.1 & 1123.4 & 984.2 & 1144.4 \\
\hline Metolong loan disbursement & $\ldots$ & $\ldots$ & $1,370.9$ & $1,370.9$ & $1,370.9$ & $1,716.6$ \\
\hline Use of Metolong loan & $\ldots$ & & 91.9 & 247.5 & 386.8 & 572.2 \\
\hline
\end{tabular}

Sources: Ministry of Finance and Development Planning; Central Bank of Lesotho; and Fund staff estimates.

${ }^{1}$ Values are cumulative from end-March 2010.

${ }^{2}$ Definitions and program adjusters are specified in the TMU.

${ }^{3}$ Excludes unused outstanding balances from the EIB loan for the Metolong dam.

${ }^{4}$ Continuous performance criteria

${ }^{5}$ New nonconcessional borrowing is limited to financing the Metolong dam.

${ }^{6}$ Includes spending on school feeding program, old age pension and HIVIAIDS 
Table 2. Lesotho: Structural Benchmarks

\begin{tabular}{|c|c|c|}
\hline Benchmarks & Test Date & Macroeconomic rationale \\
\hline \multicolumn{3}{|l|}{ I. Public Financial Management } \\
\hline $\begin{array}{l}\text { Prepare monthly monitoring reports assessing the impact of } \\
\text { modification to the IFMIS to prevent over-commitment. }\end{array}$ & End-September 2010 & $\begin{array}{l}\text { Support expenditure control and medium-term } \\
\text { fiscal consolidation }\end{array}$ \\
\hline $\begin{array}{l}\text { Complete an audit of user access rights to the IFMIS and } \\
\text { assign user access rights on a "need-to-use" basis }\end{array}$ & End-June 2010 & $\begin{array}{l}\text { Support expenditure control and medium-term } \\
\text { fiscal consolidation }\end{array}$ \\
\hline $\begin{array}{l}\text { Submit to Cabinet a comprehensive review of all on-going } \\
\text { capital projects, assessing their desirability and make } \\
\text { recommendations on projects to be retained or eliminated }\end{array}$ & End-September 2010 & $\begin{array}{l}\text { Enhance the efficiency and quality of public } \\
\text { expenditure }\end{array}$ \\
\hline \multicolumn{3}{|l|}{ II. Structural Reforms } \\
\hline Submit to Parliament the Companies Bill & End- September 2010 & $\begin{array}{l}\text { Improve the business climate to facilitate private } \\
\text { sector-led growth, and increase productivity and } \\
\text { competitiveness }\end{array}$ \\
\hline \multicolumn{3}{|l|}{ III. Financial sector regulation } \\
\hline Issue domestic bonds & End-December 2010 & $\begin{array}{l}\text { Development of the domestic money and capital } \\
\text { markets }\end{array}$ \\
\hline Submit to Parliament the amended Financial Institutions Act & End-September 2010 & $\begin{array}{l}\text { Strengthen prudential regulations and reduce } \\
\text { systemic risks to the domestic financial sector }\end{array}$ \\
\hline
\end{tabular}




\section{Attachment III. Technical Memorandum of Understanding}

May 17, 2010

1. This memorandum sets forth the understandings between the government of Lesotho and the IMF staff regarding the definitions of the quantitative performance criteria and benchmarks for a new three-year arrangement supported under the Extended Credit Facility (ECF), as well as the respective reporting requirements. These performance criteria and benchmarks are reported in Table 1 of the government's Memorandum of Economic and Financial Policies (MEFP).

\section{A. Ceiling on the Domestic Financing Requirement (DFR) of the Central Government}

2. Definition. The central government includes the central administration and all district administrations. The domestic financing requirement of the central government is defined as net credit to the government from the banking system (that is, the Central Bank of Lesotho and the commercial banks) plus holdings of treasury bills and other government securities by the nonbank sector. For program monitoring purposes, the domestic financing requirement will be calculated as the change from end-March 2010 of net credit to the government by the banking system and of holdings of treasury bills and other government securities by the nonbank sector. In particular, the calculation of the domestic financing requirement shall include changes in (i) balances held in the privatization account or balances of other accounts into which proceeds from the sale of public enterprises are deposited; (ii) the amount of outstanding treasury bills issued by the Central Bank of Lesotho for monetary policy purposes; and (iii) the balance of the blocked government deposit account used by the Central Bank of Lesotho to sterilize reserve money absorbed by monetary policy operations. The calculation of the domestic financing requirement shall exclude changes in balances held in any account into which revenues collected by the customs department are held pending their transfer to the SACU revenue pool. External debt service, amortization, disbursements and external grants will be calculated at an exchange rate of M 8.55 per U.S. dollar.

3. Supporting material. The Central Bank of Lesotho will provide the monetary survey and other monthly monetary statistics, as well as a table showing the details of all government financing operations from the nonbank public, on a monthly basis and within 30 days of the end of the month. The following information will be presented as memorandum items in the monetary survey: (i) the outstanding balances in the privatization account or accounts; and (ii) details of any monetary operations with treasury bills, including changes in government deposits as a result of such operations. The Central Bank of Lesotho will also provide a table showing the details of government debt by type and holder. The Ministry of Finance and Development Planning will provide detailed monthly budget operation reports and tax arrears reports. 


\section{B. Ceiling on the Stock of Net Domestic Assets of the Central Bank of Lesotho}

4. Definition. The net domestic assets (NDA) of the Central Bank of Lesotho are defined as the difference between reserve money (currency in circulation plus total bank deposits at the central bank) and NFA (as defined in paragraph 5). The NDA thus includes net claims by the Central Bank of Lesotho on the government (loans and treasury bills purchased less government deposits), claims on banks, and "other items net" (other assets, other liabilities, and the capital account).

5. Definition. The net foreign assets (NFA) of the Central Bank of Lesotho are defined as foreign assets minus foreign liabilities, and include all foreign claims and liabilities of the central bank. The values of all foreign assets and liabilities will be calculated in U.S. dollars at the end of each quarter using the program exchange rates.

6. Supporting material. The Central Bank of Lesotho will provide detailed data on its balance sheet on a monthly basis within 21 days of the end of the month. The central bank will also provide a table of selected monetary indicators covering the major elements of its balance sheet on a weekly basis.

\section{Floor on the Stock of Net International Reserves of the Central Bank of Lesotho}

7. Definition. The net international reserves (NIR) are defined as the Central Bank of Lesotho's liquid, convertible foreign assets minus its convertible foreign liabilities. Pledged or otherwise encumbered assets, including, but not limited to, assets used as collateral or as guarantee for third-party external liabilities are excluded from reserve assets. Reserve assets include cash and balances held with banks, bankers' acceptances, investments, foreign notes and coins held by the Central Bank of Lesotho, Lesotho's reserve position in the Fund, and SDR holdings. Reserve liabilities include nonresident deposits at the Central Bank of Lesotho, use of IMF credit, and any other liabilities of the central bank to nonresidents. The stock of NIR at the end of each quarter is defined in U.S. dollars and will be calculated using the program exchange rates. ${ }^{1}$

8. Supporting material. The Central Bank of Lesotho will provide data on its NIR on a monthly basis within one week of the end of the month. The NIR data will be provided in a

\footnotetext{
${ }^{1}$ Program cross exchange rates are: South African rand per U.S. dollar: 7.; U.S. dollars per pound sterling: 1.5; U.S. dollars per euro: 1.3; Swiss francs per U.S. dollar: 1.1; Swedish kronor per U.S. dollar: 7.3; and Botswana pula per U.S. dollar: 6.8. Program maloti per U.S. dollar exchange rate: 7.3.
} 
table showing the currency breakdown of the reserve assets and reserve liabilities of the Central Bank of Lesotho converted into U.S. dollars and maloti at the program exchange rates.

\section{Ceiling on the Amount of New Nonconcessional External Debt Contracted or Guaranteed by the Public Sector, with Original Maturity of One Year or More}

9. Definition. For purposes of the ECF, concessionality requirements will be applied to foreign-currency denominated debt regardless of the residency of the creditor. The public sector comprises the central government, the Central Bank of Lesotho, and all public enterprises and other official sector entities with majority state ownership. This performance criterion applies not only to debt as defined in point No. 9 of the Guidelines on Performance Criteria with Respect to External Debt in Fund Arrangements, adopted by Decision No. 6230-(79/140), as revised on August 24, 2000, as amended effective December 1, 2009, but also to commitments contracted or guaranteed for which value has not been received. Included in this performance criterion are all current liabilities that are created under a contractual arrangement through the provision of value in the form of assets (including currency) or services, and that require the public sector (obligor) to make one or more payments in the form of assets (including currency) at some future point(s) in time to discharge principal and/or interest liabilities incurred under the contract. In effect, all instruments that share the characteristics of debt as described above (including loans, suppliers' credits, and leases) will be subject to the ceiling. New nonconcessional borrowing is limited to the Metolong dam, a high-return public investment project. The performance criterion will be evaluated on a continuous basis as the cumulative change in the amount of new nonconcessional debt contracted or guaranteed from end-March 2010 onward.

10. Definition. A loan is concessional if its grant element is at least 35 percent of the value of the loan, calculated using a discount rate based on commercial interest reference rates (CIRRs) reported by the OECD. For loans of maturity of greater than 15 years, the grant element will be based on the ten-year average of OECD CIRRs. For loans of maturity of 15 years or less, the grant element will be based on the six-month average of OECD CIRRs. Margins for differing repayment periods would be added to the CIRRs: 0.75 percent for repayment periods of less than 15 years, 1 percent for repayment periods of 15 to 19 years, 1.15 percent for repayment periods of 20 to 29 years, and 1.25 percent for repayment periods of 30 years or more.

11. Supporting material. Details of all new commitments and government guarantees for external borrowing, with detailed explanations, will be provided by the Ministry of Finance and Development Planning on a monthly basis within 30 days of the end of the month. 


\section{E. Ceiling on the Amount of New External Debt Contracted or Guaranteed by the Public Sector, with Original Maturity of Less than One Year}

12. Definition. The public sector comprises the central government, the Central Bank of

Lesotho, and all enterprises with majority state ownership. This performance criterion applies not only to debt as defined in point No. 9 of the Guidelines on Performance Criteria with Respect to External Debt in Fund Arrangements, adopted by Decision No. 6230-(79/140), as revised on August 24, 2000, as amended effective December 1, 2009, but also to commitments contracted or guaranteed for which value has not been received. Included in this performance criterion are all current liabilities that are created under a contractual arrangement through the provision of value in the form of assets (including currency) or services, and that require the public sector (obligor) to make one or more payments in the form of assets (including currency) at some future point(s) in time to discharge principal and/or interest liabilities incurred under the contract. In effect, all instruments that share the characteristics of debt as described above (including loans, suppliers' credits, and leases) will be subject to the ceiling. Excluded from this performance criterion are normal short-term import credits. The performance criterion will be evaluated on a continuous basis as the cumulative change in the amount of new nonconcessional debt contracted or guaranteed from end-March 2010.

13. Supporting material. Details of all new commitments and government guarantees for external borrowing, with detailed explanations, will be provided by the Ministry of Finance and Development Planning on a monthly basis within 30 days of the end of the month.

\section{F. Ceiling on the Stock of External Payments Arrears}

14. Definition. During the period of the arrangement, the stock of external payments arrears of the public sector (central government, Central Bank of Lesotho, and all enterprises with majority state ownership) will continually remain zero. Arrears on external debt-service obligations include any nonpayment of interest and/or principal in full and on time falling due to all creditors, including the IMF and the World Bank.

15. Supporting material. Details of arrears accumulated on interest and principal payments to creditors will be reported within one week from the date of the missed payment.

\section{G. Ceiling on Domestic Arrears}

16. Definition: Domestic arrears are overdue payment obligations of the central government other than external payment arrears, including on wages and salaries, pensions, transfers, domestic interest, goods and services, obligations arising from court cases, legally established compensation claims, and tax refunds. Payments on wages and salaries, pensions, transfers, court-established obligations, and compensations are in arrears when they remain 
unpaid for more than 45 days beyond their due date. Domestic interest payments are in arrears when the payment is not made on the due date. Payments for goods and services are deemed to be in arrears if they have not been made within 45 days of the date of invoice, or-if a grace period has been agreed—within the contractually agreed grace period.

17. Supporting material: Information regarding domestic arrears is to be compiled through audits of the accounts of spending Ministries and agencies, conducted by the Internal Audit division of the Ministry of Finance and Development Planning. The audits will be completed and data submitted to IMF staff within six weeks of the end of each quarter. This audit information will be cross-checked with the data obtained from the IFMIS with a view to improving the functionality of the IFMIS.

\section{H. Floor on the Central Government Social Expenditures}

18. Definition: There will be a floor on the Central Government Social Expenditures. The observance of this floor is an indicative target. Social expenditures comprise spending on the following programs: school feeding program, old age pension, and HIV/AIDS.

\begin{tabular}{|c|c|c|c|c|}
\hline \multicolumn{5}{|c|}{$\begin{array}{l}\text { Social Expenditures 2010/11 } \\
\text { (in millions of Maloti) }\end{array}$} \\
\hline & Jun-10 & Sep-10 & Dec-10 & Mar-11 \\
\hline Social Expenditures & 170.0 & 170.0 & 170.0 & 170.0 \\
\hline Social Benefits & 88.8 & 88.8 & 88.8 & 88.8 \\
\hline$o / w$ Old Age Pension & 73.8 & 73.8 & 73.8 & 73.8 \\
\hline APC Pension & 5.5 & 5.5 & 5.5 & 5.5 \\
\hline Public Assistance Cash \& Kind & 9.5 & 9.5 & 9.5 & 9.5 \\
\hline School Feeding Program & 54.5 & 54.5 & 54.5 & 54.5 \\
\hline HIV/AIDS & 26.7 & 26.7 & 26.7 & 26.7 \\
\hline$o / w$ Anti-Retroviral Therapies & 23.7 & 23.7 & 23.7 & 23.7 \\
\hline
\end{tabular}

19. Supporting material: Data on social spending will be compiled by the Ministry of Finance and Development Planning and will be provided on a quarterly basis, to be submitted no later than six weeks after the end of each reporting period.

\section{Adjusters}

20. The quantitative performance criteria specified under the program are subject to the following adjusters: 


\section{A. Southern African Customs Union Revenues}

The program targets for the NIR, NDA, and DFR in any quarter will be adjusted upward by the amount of any advance receipts from the Southern Africa Customs Union (SACU) in that quarter, where such advance receipts constitute amounts that would otherwise have been received in a subsequent quarter. It will be adjusted for accounting practice changes implemented by the Central Bank of Lesotho that are recommended by the IMF's Statistics Department or are made in response to the IMF safeguards assessment.

21. Supporting material: The Central Bank of Lesotho will provide data on SACU receipts on a quarterly basis within the first month of the quarter.

\section{B. Budgetary Support net of Debt Service}

The ceilings on NDA and DFR will be adjusted downward (upward) by the full amount of the excess (shortfall) in budgetary support net of debt service relative to the programmed levels specified in Table 1 of the MEFP.

The floor on the NIR of the Central Bank of Lesotho will be adjusted upward (downward) by the full amount of the excess (shortfall) in budgetary support net of debt service relative to the programmed levels specified in Table 1 of the MEFP.

22. Supporting material: Data on budget support and debt service will be compiled by the Ministry of Finance and Development Planning and will be provided on a quarterly basis, to be submitted no later than six weeks after the end of each reporting period.

\section{Payment of Arrears}

The ceilings on NDA and DFR will be adjusted downward (upward) by the full amount of the excess (shortfall) in payments of arrears relative to the programmed levels specified in Table 1 of the MEFP.

The floor on the NIR of the Central Bank of Lesotho will be adjusted downward (upward) by the full amount of the excess (shortfall) in payment of arrears relative to the programmed levels specified in Table 1 of the MEFP.

23. Supporting material: Data on domestic arrears will be compiled by the Ministry of Finance and Development Planning and will be provided on a quarterly basis, to be submitted no later than six weeks after the end of each reporting period.

\footnotetext{
${ }^{2}$ General budget support consists of grants and loans received by the Central Government for financing its overall policy and budget priorities.
} 


\section{INTERNATIONAL MONETARY FUND}

\section{KINGDOM OF LESOTHO}

\section{Request for a Three-Year Arrangement Under the Extended Credit Facility- Informational Annex}

Prepared by the African Department in Consultation with Other Departments

Approved by Domenico Fanizza and Tom Dorsey

May 17, 2010

- Relations with the Fund. Describes financial and technical assistance by the IMF, and provides information on the exchange rate system. Lesotho's last Fund arrangement, a PRGF, expired in 2004.

- The JMAP Bank-Fund. Describes the priorities and main activities of the World Bank Group and the IMF, and areas of cooperation in their work with the authorities of Lesotho.

- $\quad$ Statistical Issues. Assesses the quality of statistical data. Economic data are adequate for surveillance purposes, though some deficiencies remain. Lesotho subscribes to the GDDS. 


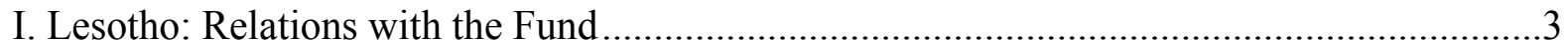

II. Lesotho: The JMAP Bank-Fund Matrix....................................................................6

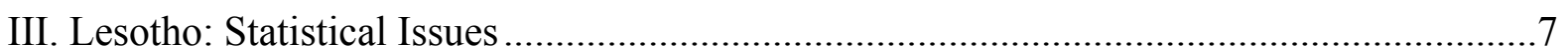




\section{LESOTHO: RELATIONS WITH THE FUND}

(As of March 31, 2010)

I. Membership status: Joined 07/25/1968; accepted the obligations of Article VIII, Sections 2, 3, and 4: 03/05/1997.

II. General resources account:

Quota

Fund holdings of currency

Reserve position in Fund

III. SDR Department:

Net cumulative allocation

Holdings

IV. Outstanding purchases and loans:

$\mathrm{ECF}^{1}$ arrangements $\underline{\text { SDR Million }}$

34.90

31.32

3.61

$\underline{\text { SDR Million }}$

32.88

29.16

$\underline{\text { SDR Million }}$

13.30

38.11

V. Financial arrangements:

$\begin{array}{ccccc}\text { Type } & \begin{array}{c}\text { Approval } \\ \text { Date }\end{array} & \begin{array}{c}\text { Expiration } \\ \text { Date }\end{array} & \begin{array}{c}\text { Amount Approved } \\ \text { (SDR Million) }\end{array} & \begin{array}{c}\text { Amount Drawn } \\ \text { (SDR Million) }\end{array} \\ \text { ECF } & 03 / 09 / 2001 & 10 / 31 / 2004 & 24.50 & 24.50 \\ \text { Stand-By } & 09 / 23 / 1996 & 9 / 22 / 1997 & 7.17 & 0.0 \\ \text { Stand-by } & 07 / 31 / 1995 & 7 / 30 / 1996 & 7.17 & 0.0\end{array}$

\footnotetext{
${ }^{1}$ Formerly PRGF
} 
VI. Projected obligations to fund (SDR million; based on existing use of resources and present holdings of SDRs):

\begin{tabular}{lllll}
\multicolumn{5}{c}{ Forthcoming } \\
\hline$\underline{2010}$ & $\underline{2011}$ & $\underline{2012}$ & $\underline{2013}$ & $\underline{2014}$ \\
2.80 & 4.55 & 3.15 & 1.75 & 1.05 \\
0.01 & 0.01 & 0.02 & 0.02 & 0.01 \\
$\mathbf{2 . 8 1}$ & $\mathbf{4 . 5 6}$ & $\mathbf{3 . 1 7}$ & $\mathbf{1 . 7 7}$ & $\mathbf{1 . 0 6}$
\end{tabular}

VII. Under the Fund's safeguards assessment policy, the CBL was subject to a safeguards assessment with respect to $\mathrm{ECF}^{1}$ arrangement approved on March 09, 2001. The assessment, completed on July 24, 2003, found that while the bank has made notable progress in strengthening its safeguards since the off-site assessment in July 2001, vulnerabilities remain, in particular in the areas of internal audit and internal controls.

\section{Exchange arrangement:}

Lesotho's currency, the loti (plural maloti), is pegged to the South African rand at par. Both the loti and rand are legal tenders. As of May 5, 2010, the maloti rate per U.S. dollar was M 7.64.

\section{Article IV consultation:}

The 2009 Article IV consultation was concluded by the Executive Board on February 22, 2010. Lesotho is on the standard 12-month Article IV consultation cycle.

\section{Technical assistance missions:}

\author{
Monetary policy implementation \\ Public accounts \\ General Data Dissemination System (GDDS) project for \\ Anglophone Africa \\ Balance of payments \\ Monetary policy and AML/CFT \\ Government finance statistics \\ National accounts \\ National accounts statistics \\ GDDS project for Anglophone Africa \\ Legislative drafting/FIU \\ Monetary operations/payments issues/banking supervision \\ Government finance statistics/GDDS \\ Banking law \\ National accounts statistics/GDDS \\ Monetary operations \\ Payments issues \\ Banking law \\ BOP statistics/GDDS
}

$\begin{array}{lll}\text { MAE } & \text { short-term } & 2001 \\ \text { FAD } & \text { short-term } & 2002 \\ \text { STA } & \text { short-term } & 2002 \\ & & \\ \text { STA } & \text { short-term } & 2002 \\ \text { MFD } & \text { short-term } & 2003 \\ \text { STA } & \text { short-term } & 2003 \\ \text { STA } & \text { short-term } & 2003 \\ \text { STA } & \text { short-term } & 2004 \\ \text { STA } & \text { short-term } & 2004 \\ \text { LEG } & \text { Short-term } & 2005 \\ \text { MFD } & \text { short-term } & 2005 \\ \text { STA } & \text { short-term } & 2005 \\ \text { LEG } & \text { short-term } & 2005 \\ \text { STA } & \text { short-term } & 2005 \\ \text { MFD } & \text { short-term } & 2005 \\ \text { MFD } & \text { short-term } & 2005 \\ \text { LEG } & \text { short-term } & 2005 \\ \text { STA } & \text { short-term } & 2005\end{array}$


GDDS consultation

STA short-term 2005

Government Finance Statistics: GDDS Project for Anglophone

STA short-term

2006

African Countries

Payment System Reform

LEG short-term 2006

Central Banking and Banking Law

LEG short-term 2006

Bank Supervision/Monetary Operations/Payments \& Settlements

MFD short-term 2006

Legal drafting, AML/CFT, FIU, supervisory and institutional

LEG short-term 2006

Regional workshop on the statistical treatment of SACU transfers STA short-term 2007

Monetary and Financial Statistics

STA short-term 2007

Nonbank supervision

Bank supervision

Monetary operations/Bank supervision

Payment systems

Government Finance Statistics: GDDS Project for Anglophone

MCM short-term

2007

MCM short-term 2007

MCM short-term 2007

MCM short-term 2007

African Countries

Money markets

Banking law and regulation

Balance of payment

Payment systems modernization

Bank supervision

Payment systems

Money markets

Monetary and Financial Statistics

STA short-term

2008

MCM short-term 2008

LEG short-term 2008

STA short-term 2008

MCM Short-term 2008

MCM short-term 2008

MCM short-term 2009

MCM short-term 2009

STA short-term 2009

Missions planned for $\mathbf{2 0 1 0}$

Balance of Payments

Public Financial Management

STA short-term 2010

Financial sector regulations

FAD short-term 2010

LEG short-term 2010

\section{Resident Representatives:}

None. 


\section{LESOTHO: THE JMAP BANK-FUND MATRIX}

(As of April 6, 2010)

\begin{tabular}{|c|c|c|c|}
\hline Title & Products & $\begin{array}{c}\text { Provisional Timing of } \\
\text { missions }\end{array}$ & $\begin{array}{l}\text { Expected Delivery } \\
\text { date }\end{array}$ \\
\hline \multicolumn{4}{|c|}{ A. Mutual information on relevant work programs } \\
\hline $\begin{array}{l}\text { Bank work } \\
\text { program in next } \\
12 \text { months }\end{array}$ & $\begin{array}{l}\text { - CAS } \\
\text { - ESW on Regional Trade and } \\
\text { Integration } \\
\text { - Third Poverty Reduction Support } \\
\text { Credit } \\
\text { - Private Sector competitiveness and } \\
\text { Economic Diversification project } \\
\text { - Post Basic Education ESW }\end{array}$ & $\begin{array}{l}\text { - February } 2010 \\
\text { - Ongoing } \\
\text { - August } 2010 \\
\text { - Ongoing regular } \\
\text { missions } \\
\text { - January } 2010\end{array}$ & $\begin{array}{l}\text { - June } 2010 \\
\text { - November } 2010 \\
\text { - April } 2011 \\
\text { - Project effective, } \\
\text { implementation } \\
\text { ongoing } \\
\text { - } 2011\end{array}$ \\
\hline $\begin{array}{l}\text { IMF work } \\
\text { program in next } \\
12 \text { months }\end{array}$ & $\begin{array}{l}\text { - Program review and } 2010 \text { Article IV } \\
\text { Article IV Consultation }\end{array}$ & - November 2010 & - February 2011 \\
\hline \multicolumn{4}{|c|}{ B. Requests for work program inputs } \\
\hline $\begin{array}{l}\text { Fund request to } \\
\text { Bank }\end{array}$ & $\begin{array}{l}\text { - Assisting the authorities with food } \\
\text { strategy and scaling up agricultural } \\
\text { yield. } \\
\text { - Updates on: private sector } \\
\text { competitiveness and development, } \\
\text { PPP capacity building, SMEs } \\
\text { financing schemes and } \\
\text { decentralization issues } \\
\text { - Updates on budget support programs }\end{array}$ & $\begin{array}{l}\text { - Ongoing } \\
\text { - Ongoing } \\
\text { - Ongoing }\end{array}$ & \\
\hline $\begin{array}{l}\text { Bank request to } \\
\text { Fund }\end{array}$ & $\begin{array}{l}\text { - Data and macro framework (including } \\
\text { the underlying analytical model). } \\
\text { - Report on the Fund TA on IFMIS }\end{array}$ & $\begin{array}{l}\text { - Regularly } \\
\text { - Regularly }\end{array}$ & \\
\hline \multicolumn{4}{|c|}{ C. Agreements on joint products and missions } \\
\hline $\begin{array}{l}\text { Joint products } \\
\text { in next } 12 \\
\text { months }\end{array}$ & Low income debt sustainability analysis & $\begin{array}{l}\text { - March } 2010 \\
\text { - November } 2010\end{array}$ & $\begin{array}{l}\text { - May } 2010 \\
\text { - February } 2011\end{array}$ \\
\hline
\end{tabular}




\section{Lesotho: Statistical ISSUES}

The statistical database is adequate for surveillance, although there are some deficiencies in core surveillance data, including government finance and balance of payments statistics that are currently being addressed by the authorities.

As one of 22 countries participating in the GDDS Project for Anglophone African Countries (funded by the U.K. Department for International Development (DFID)), Lesotho has undertaken the GDDS as framework for the development of its national statistical system and a depository of metadata for main macroeconomic aggregates. GDDS metadata have been posted on the Fund's Dissemination Standards Bulletin Board since August 2003. Pending metadata updates in GDDS include those referring to recent/prospective revisions of the national accounts, government finance, and balance of payments statistics.

\section{National accounts}

Revised national accounts were released in November 2008 by the Bureau of Statistics of Lesotho (BSL). Technical assistance from the EU and DFID supported the data revision and the rebasing of the GDP data from 1981 to 2007. Important new sources of data include the use of VAT records and consumer spending information from the 2002/03 household budget survey. Annual GDP estimates in current prices and volume terms from the production, expenditure, and income sides are currently being produced and published by the BLS, albeit with different time lags. Other macroeconomic indicators, such as highfrequency industrial production/trade indices, are used to monitor developments during the year.

There is a need to further improve the source data used for compiling national accounts. Pending challenges include, inter alia, robust data on intermediate consumption for key economic activities, intra-SACU exports and imports, and gross fixed capital formation in machinery and equipment, and formal and informal construction activities. Private consumption and gross fixed capital formation are now estimated within the framework of a supply and use table. However, it has not been possible to balance supply and use completely, hence the remaining discrepancy between the tables on GDP by activity and expenditure.

\section{Prices}

The consumer price index (CPI) is estimated on a monthly basis by the Bureau of Statistics (BOS). The CPI is designated to show changes in the cost of buying a fixed bundle of consumer goods and services as determined for the base period (January 2004) using spending weights from the 2002/03 Household Budget Survey (HBS). Prices are collected every month in twelve urban towns and forty five selected rural areas of Lesotho by personal visits to outlets. 
Plans are in place at the BOS to develop producer and export price indices. In the meantime, the national accounts rely heavily on the South African producer price index (PPI), which is used as a deflator for imports from SACU member countries. South Africa's import price indices are used as deflators for imports from non-SACU countries.

\section{Government finance statistics}

Data on government finance statistics are provided by the Ministry of Finance and Development Planning (MoFDP) to AFR periodically, and the timeliness and quality of the data have improved. Technical assistance from STA and DIMMOL supported migration of data from GFSM 1986 to the GFSM 2001 framework. AFR has been provided with monthly, quarterly and annual disaggregated central government data (on a cash basis) for the period 1991/92-2008/09, using the GFSM 2001 framework. These statistics were compiled from the GOLFIS, supplemented with data from the Lesotho Revenue Authority (LRA), other government departments and units and the banking system.

The Integrated Financial Management Information System (IFMIS), introduced in April 2009, and which has modules consistent with the GFSM 2001 framework, is now the main source of government finance statistics. However, as a result of teething problems experienced with the new IFMIS, there has been inadequate data capture of revenues and budget execution data for 2009/10, and therefore shortcomings to the data to prepare the 2010/11 budget. To ensure timely reconciliation of and monitoring of budget execution, the IFMIS implementation issues need to be addressed urgently, including ensuring proper reconciliation and adequate quality check of data from line ministries and government units. The authorities have integrated the government payroll system (Unique, implemented in September 2009) with the IFMIS and are working to integrate the CS-DRMS - the public debt recording and management system and the LRA system - the VIPS. There are also plans to interface IFMIS with the CBL.

The MFDP also provides annual data to STA on the operations of the general government for publication in the Government Finance Statistics Yearbook. No high frequency data are reported for publication in the International Financial Statistics. The MFDP disseminates government finance statistics, including government debt, to the public through its website and the website of the Bureau of Statistics.

\section{Monetary and financial statistics}

Since December 2004, Lesotho has been compiling monetary statistics based on the Standardized Report Forms (SRFs), which allow for an improved classification and sectorization of accounts. Monthly data using SRFs are currently disseminated in the International Finance Statistics Supplement.

Despite the progress achieved, the authorities need to expand the institutional coverage of the other depository corporations sector. A monetary and financial statistics mission in 
September 2007 found that work had started on the inventory of all financial corporations (deposit-taking and others), and that it was expected that credit cooperatives would be fully covered in the other depository corporations survey in the last quarter of 2008. The compilation of the other financial corporations' survey had been targeted for 2009.

\section{Balance of payments and external debt}

The CBL compiles quarterly balance of payments statistics, usually with a lag of three months. However, a number of methodological and data classification issues with current practices will need to be addressed to make the national presentation consistent with the standard presentation recommended in the IMF's Balance of Payments Manual, fifth edition (BPM5).

\section{Balance of payments missions from the IMF Statistics department have been advising the authorities on methodological and data issues since 2002. A STA mission in mid-2002 found that the methodology underlying the balance of payments compilation used outdated benchmark surveys, had an incomplete coverage of main economic activities (e.g., commodity exports and imports, services, and direct investment statistics), and depended on data sources that were not produced on a timely basis.}

A follow-up STA mission in July/August 2008 made further recommendations on data improvements. The mission welcomed the CBL efforts to implement some new data surveys -on private capital flows, road transportation, telecommunications, postal and courier services, and remittances - which extended the data coverage and established benchmarks in data compilation. However, the quality of the balance of payments still has inadequacies, in large part due to undercoverage and the erroneous treatment of some transactions. With the full support of the compilers, the mission reviewed the sources and methods employed in compiling the balance of payments and the International Investment Position (IIP) and found a number of ways to strengthen them, especially in the area of coverage. The mission emphasized the need to continue conducting the private capital flows survey each year, with a particular focus on increasing the coverage of foreign direct investment enterprises and the survey's response rate. On dissemination practices, the mission urged the authorities to post the balance of payments and IIP data at the CBL website on a quarterly basis.

The authorities are currently implementing the technical assistance recommendations provided by the IMF. The first step has been to implement methodological changes that do not require data sources, but would ensure correct and consistent use of data classifications along the BPM5. Subsequent steps will include, notably, improved estimates of imports of goods and services.

Data on public sector external debt is provided by the MFDP. The MoFDP maintains a comprehensive database of outstanding loans, with information on currency composition, debt service and amortization, as well as scheduled future disbursements. 


\section{Lesotho: Table of Common Indicators Required for Surveillance}

(As of April 16, 2010)

\begin{tabular}{|c|c|c|c|c|c|}
\hline & $\begin{array}{c}\begin{array}{c}\text { Date of } \\
\text { latest } \\
\text { observation }\end{array} \\
\end{array}$ & $\begin{array}{l}\text { Date } \\
\text { received }\end{array}$ & $\begin{array}{c}\text { Frequency } \\
\text { of } \\
\text { Data } 7 / \\
\end{array}$ & $\begin{array}{c}\text { Frequency } \\
\text { of } \\
\text { Reporting } 7 /\end{array}$ & $\begin{array}{l}\text { Frequency of } \\
\text { Publication } 7 /\end{array}$ \\
\hline Exchange rates & April 2010 & April 2010 & $\mathrm{D}$ & M & $\mathrm{D}$ \\
\hline $\begin{array}{l}\text { International reserve assets and reserve liabilities } \\
\text { of the monetary authorities } 1 /\end{array}$ & April 2010 & April 2010 & M & Q & Q \\
\hline Reserve/base money & Feb. 2010 & April 2010 & M & Q & Q \\
\hline Broad money & Feb. 2010 & April 2010 & M & Q & Q \\
\hline Central bank balance sheet & Feb. 2010 & April 2010 & M & Q & Q \\
\hline Consolidated balance sheet of the banking system & Feb. 2010 & April 2010 & M & Q & Q \\
\hline Interest rates $2 /$ & Feb. 2010 & April 2010 & M & M & M \\
\hline Consumer price index & Nov. 2009 & Dec. 2009 & M & M & M \\
\hline $\begin{array}{l}\text { Revenue, expenditure, balance and composition of } \\
\text { financing } 3 / \text {-general government } 4 /\end{array}$ & $\mathrm{N} / \mathrm{A}$ & $\mathrm{N} / \mathrm{A}$ & N/A & N/A & $\mathrm{N} / \mathrm{A}$ \\
\hline $\begin{array}{l}\text { Revenue, expenditure, balance and composition of } \\
\text { financing } 3 / \text {-central government }\end{array}$ & Feb. 2010 & March 2010 & M & I & Q \\
\hline $\begin{array}{l}\text { Stocks of central government and central } \\
\text { government-guaranteed debt } 5 /\end{array}$ & Feb. 2010 & March 2010 & M & 1 & Q \\
\hline External current account balance & Q4 2009 & March 2010 & Q & Q & Q \\
\hline Exports and imports of goods and services & Q4 2009 & March 2010 & Q & Q & Q \\
\hline GDP/GNP & 2009 & March 2010 & A & $A$ & A \\
\hline Gross external debt & Feb. 2010 & April 2010 & M & Q & Q \\
\hline International investment position $6 /$ & Q4 2008 & Dec., 2009 & Q & Q & Q \\
\hline
\end{tabular}

$1 /$ Includes reserve assets pledged or otherwise encumbered as well as net derivative positions.

2/ Both market-based and officially-determined, including discount rates; money market rates; and rates on treasury bills, notes, and bonds.

3/ Foreign, domestic bank, and domestic nonbank financing.

4/ The general government consists of the central government (budgetary funds, extra budgetary funds, and social security

funds) and state and local governments.

$5 /$ Including currency and maturity composition.

6/ Includes external gross financial asset and liability positions vis-à-vis nonresidents.

7/Daily (D), weekly (W), monthly (M), quarterly (Q), annually (A), irregular (I); and not available. 


\section{INTERNATIONAL MONETARY FUND AND \\ INTERNATIONAL DEVELOPMENT ASSOCIATION}

KINGDOM OF LESOTHO

\section{Joint World Bank/IMF Debt Sustainability Analysis}

Prepared by the staffs of the International Monetary Fund and The International Development Association

Approved by Domenico Fanizza and Tom Dorsey (IMF)

May 17, 2010

This debt sustainability analysis for low-income countries (LIC DSA) updates the one completed and presented to the IMF Board, on February 22, 2010, together with the 2009 Article IV Staff Report (SM/10/31, SUP. 1). A key revision is the incorporation of a new nonconcessional external loan for construction of the Metolong dam. As a result, Lesotho's risk of debt distress has risen, but the PV of debt to GDP ratio is still projected to return to a manageable level over the medium term as the fiscal position improves. If the significant amount of remittances to Lesotho is taken into account, the modified debt ratios decline significantly and the risk of debt distress is substantially reduced to moderate. Lesotho remains vulnerable to adverse shocks to the exchange rate or real GDP growth, as well as to a permanent decline in the level of SACU receipts. These results underscore the need to curtail additional nonconcessional borrowing, realign spending with its sustainable long-run level of SACU revenues, and accelerate structural reforms to boost productivity and competitiveness in order to accelerate medium-term growth. 


\section{INTRODUCTION}

\section{This updated DSA has been prepared by the IMF staff as an annex to the} request for a three-year ECF arrangement. The DSA comprises external and domestic debt, and is based on the framework for low-income countries approved by the respective Executive Boards. Given the importance of remittances in enhancing Lesotho's capacity to repay debt, the DSA uses the remittance-modified debt indicators to assess the risk of debt distress. ${ }^{1}$ The framework takes into account indicative thresholds for debt burden indicators determined by the quality of the country's policies and institutions, ${ }^{2}$ and comprises baseline and alternative scenarios.

\section{Lesotho's nominal public sector debt declined from 52 percent of GDP at end-} 2008 to 40 percent at end-2009, mainly because of a significant (21 percent) appreciation of the loti/dollar exchange rate. Of the total public sector debt, US\$664 million was owed to external creditors, almost entirely to multilaterals (US\$611 million), mainly IDA and the African Development Fund. Public domestic debt (held by residents) amounted to US\$72 million at the end of 2009; at present, domestic debt is limited to Treasury bills issued for liquidity management and is not used for budget financing.

\footnotetext{
${ }^{1}$ See "Applying the Debt Sustainability Framework for Low-Income Countries Post Debt Relief," (IDA/SecM2006-0564 and SM/07/131) and "Staff Guidance Note on the Application of the Joint Fund-Bank Debt Sustainability Framework for Low-Income Countries," (SM/10/16).

${ }^{2}$ The World Bank Country Policy and Institutional Assessment has ranked Lesotho using the three-year moving average as a "medium performer" in terms of policy and institutions with a rating of 3.53. The applicable indicative thresholds for debt sustainability (DSF), proposed under the framework for low-income countries are: (i) 40 percent for the NPV of debt-to-GDP ratio, (ii) 150 percent for NPV of debt-to-exports ratio; (iii) 250 percent for the NPV of debt-to-fiscal revenues ratio; (iv) 20 percent for the debt service to exports ratio; and (v) 30 percent for the debt service to revenue ratio.
} 
Lesotho: Public Debt Outstanding at end-2009

\begin{tabular}{lrr}
\hline & $\begin{array}{c}\text { In Millions } \\
\text { of USD }\end{array}$ & $\begin{array}{c}\text { In Percent of } \\
\text { GDP }\end{array}$ \\
\hline Total public debt & 736.5 & 40.2 \\
Domestic debt & 72.2 & 3.9 \\
External debt & 664.3 & 36.3 \\
Multilateral & 611.1 & 33.4 \\
World Bank Group & 317.7 & 17.0 \\
African Development Fund & 215.9 & 11.6 \\
EU & 25.7 & 1.6 \\
IMF & 23.6 & 1.5 \\
Others & 28.2 & 1.7 \\
Bilateral & 45.7 & 2.4 \\
Commercial & 7.5 & 0.4 \\
\hline
\end{tabular}

Source: Ministry of Finance and Development Planning

3. The large decline in SACU revenues projected for 2010/11 remains the key driver of fiscal and external outlook as well as future debt dynamics. The resulting increase in public debt will depend on the extent to which the authorities are able to implement offsetting expenditure measures and mobilize financing from donors on concessional terms. While the level of SACU revenues is expected to increase as South Africa's economy recovers, it is unlikely to return to the very high levels recorded in recent years. In addition, there is considerable uncertainty regarding the level of SACU revenues over the longer term, with the possibility of revisions to the revenue-sharing formula that would lower the share of the Common Revenue Pool (CRP) allocated to the BLNS countries.

\section{Macroeconomic Assumptions}

\section{The underlying macroeconomic assumptions for this DSA differ from those of} the February 2010 DSA in the following respects: The fiscal and external account deficits are wider, but the average real GDP growth rate (2010-15) and debt outstanding are higher as a result of debt-financed spending for the Metolong dam project and the proposed Fund disbursements.

- $\quad$ Output growth is now expected to average 5.7 percent annually during 2010-15, boosted by construction of the Metolong dam (starting in 2010/11), as against 5.3 percent previously. Initial construction of the LHWPII is expected to begin in 2012, with the largest expenditures taking place during 2015-19. Growth in 2015 is projected to reach 12.1 percent due to the impact of the LHWPII.

- Inflation (as measured by the implicit GDP deflator) is assumed to move from an average of 7.7 percent over 2000-09 (elevated in part by the food and energy 
price shocks in 2007-08) to 3 percent over the longer term, in line with projected consumer price inflation in South Africa.

- $\quad$ The average fiscal deficit has widen by 3.5 percent of GDP to an annual average deficit of 14.6 percent of GDP during 2010/11-2012/13, owing to higher debtfinance capital spending related to the Metolong dam project.

- $\quad$ The profile of SACU repayments has been revised to reflect two repayments in 2010/11 and 2011/12 rather than the previous assumption of three payments stretching to $2012 / 13$. The fiscal position is projected to return to a surplus of 1.3 percent of GDP in 2015/16 compared with a balanced position in the previous DSA, in line with a sharper reduction in expenditure. Like in the previous DSA, this one assumes that the deficits will be financed by combination of drawing down government deposit with the CBL, donors' budget support and new borrowings, including domestic debt issuance.

- $\quad$ The DSA prepared for the Article IV assumed new bilateral borrowing of US\$120 million during 2011-12 to offset the SACU shortfall, at an interest rate of 6 percent and maturity of 10 years. The current DSA does not make such an assumption. Instead, the authorities intend to use their deposits to cover the revenue shortfall.

- $\quad$ The current account balance (including official transfers) which broadly reflects changes in the fiscal position has widened by 5 percent of GDP to an average of 21 percent of GDP during 2010-12, reflecting projected higher imports related to the Metolong dam. However, by 2014 the external current deficit narrows to about the same level as in the previous DSA.

- $\quad$ The DSA assumes Fund access amounting to 120 percent of quota which is equivalent to 3.4 percent of GDP (2010). The proposed access would raise the PV of debt to GDP by an average of about 2 percent of GDP during the period 2010-15. The debt service ratios would remain broadly the same (averaging 4.6 percent during 2010-15) in the scenario with or without Fund disbursement. Given the concessional nature of Fund lending, the proposed access is consistent with Lesotho's debt sustainability.

\section{EXTERNAL DEBT SUSTAINABILITY}

\section{Baseline}

\section{Lesotho's PV of external debt stood at 28.3 percent of GDP in 2010 (Table 1a)}

and is projected to rise to 42.2 percent by 2015 , above the 40 percent indicative threshold, mainly because of the new nonconcessional borrowing for the Metolong dam. This breach of the threshold lasts for 9 years, before declining to 15.3 percent by 2030 . The PV of debt 
relative to exports and to revenues would also rise through 2020, but remain well below the indicative thresholds. There would also be a modest increase in the ratio of debt service to exports (from 4.2 to 7.3 percent) and debt service to revenues (from 4.4 to 7.2 percent), but debt service ratios would remain well below the thresholds due to the highly concessional nature of existing debt.

\section{Lesotho's debt ratios fall considerably if remittances (mainly from Basotho} working in South Africa) are also included in the assessment (Figures 3-4). Specifically, the PV of external debt to the sum of GDP and remittances would reach about 34 percent by 2015 , slightly lower than the modified threshold of 36 percent, and fall thereafter to 12.3 percent by 2030. Similarly, the PV of external debt to exports and gross remittances peaks at 61.3 percent in 2015 , well below the modified threshold of 135 percent. $^{3}$

\section{Alternative scenarios and stress tests}

7. Sensitivity tests show that Lesotho's debt burden would increase, but still fall below the indicative threshold for PV of debt to GDP by 2030, in the event of less favorable public sector borrowing terms (Table 1 b and Figure 1). In a scenario in which the interest rate on new public sector loans is 2 percentage points higher than the baseline assumption (scenario A2), the PV of debt-to-GDP ratio reaches 47 percent in 2020, but falls to 19 percent by 2030 . In a scenario in which the key variables are set at their average of the past 10 years, Lesotho's debt ratios would be well below the baseline, reflecting the large fiscal surpluses over this period relative to the projected significant deterioration in the fiscal position.

8. The bound tests reveal that Lesotho would face the most distress if there were to be a significant terms of trade shock (scenario B3) or a large nominal depreciation (scenario B6). In a scenario with terms of trade shock in 2010-11, the PV of debt-to-GDP ratio would increase to 54 percent by 2020 and then ease to 19 percent by 2030. In the event of a one-time 30 percent depreciation of the nominal exchange rate (B6), the PV of debt-toGDP ratio would similarly increase to 60 percent by 2020 , but then fall to 21 percent by 2030.

\section{Country-specific scenario}

9. The increase in debt levels from 2010 and beyond is largely attributable to the increase in borrowing to finance the Metolong dam and the second phase of the

\footnotetext{
${ }^{3}$ For a country facing a DSF threshold of 150 percent of exports for its PV of debt, the remittance modified debt indicator should not exceed 135 percent. Similarly, where the DSF threshold is 40 percent of GDP, the remittance modified indicator should not exceed 36 percent of GDP.
} 
LHWP. ${ }^{4}$ With construction of the Metolong dam underway, an alternative scenario was performed assuming that the LHWPII does not go forward as planned. In this scenario (scenario A3), the PV of debt to GDP ratio would rise to 40 percent in 2013, but would then decline to 5 percent by 2030 .

\section{Public Sector DebT Sustainability}

\section{Baseline}

10. Domestic debt remained relatively low at the end of 2009, leaving public debt indicators very closely aligned to those of public external debt (Table 2a). Domestic debt is currently limited to Treasury bills issued by the Central Bank of Lesotho for liquidity management, and is not used for budget financing. Longer-term domestic debt issuance as a source of budget finance is expected to begin in 2010/11 and to increase over the medium term, as the government taps institutional investors that currently place almost all of their assets abroad due to the lack of domestic investment options. Borrowing rates are projected to follow interest rates in South Africa, but will depend on investors' perceptions of relative creditworthiness. Domestic debt is projected to increase to 8.2 percent of GDP by 2030, reflecting a gradual expansion of the domestic debt market as a source of budget finance.

\section{Alternative scenarios and stress tests}

\section{In the standard sensitivity tests, public sector debt ratios are most sensitive to} slower than projected growth (Table $\mathbf{2 b}$ and Figure 2). Lower long-run real GDP growth results in the PV of debt to GDP ratio rising steadily from 33 percent in 2010 to 63 percent in 2020 and 71 percent by 2030, compared with 23 percent in the baseline scenario in 2030 . This scenario illustrates the vulnerability of the debt trajectory in the event that the ongoing program of infrastructure investment and structural reforms fails to generate sufficient improvement in productivity and competitiveness needed to support the higher real GDP growth rates projected over the medium term.

\section{Country-specific scenario}

12. In a customized scenario featuring a permanent decline in SACU revenues, the ratio of public sector debt to GDP also rises. The baseline scenario already incorporates the projected decline in SACU transfers to Lesotho in 2010/11 and 2011/12 as a result of the economic downturn in South Africa and repayments owed on excess disbursements in previous years. However, the extent of the medium-term recovery in SACU transfers remains unclear, given the possibility of an adjustment to the revenue-sharing formula that could

\footnotetext{
${ }^{4}$ The staff's baseline scenario assumes disbursements related to construction of Metolong dam on the order of US\$66 million (equivalent to 3.3 percent of GDP) per year during 2011-13. In the case of LHWPII the disbursements average US\$53 million (equivalent to 2.2 percent of GDP) per year during 2013-19.
} 
reduce distributions to the BLNS or further reductions in the common external tariff that would also lower collections of the CRP. The baseline scenario assumes that Lesotho's SACU revenues recover to 20.5 percent of GDP by 2012/13 and subsequently stabilize at about 19.4 percent of GDP; the sensitivity scenario assumes that SACU revenues plateau at 15 percent of GDP, with the lower revenues only partly offset by a decline in expenditures, resulting in additional borrowing to finance the larger fiscal deficits that result. In a scenario in which SACU transfers are held at this lower level (Table 2b; scenario A4), the PV of debt to GDP ratio reaches 54 percent by 2020, and declines to 30 percent by 2030 .

\section{CONCLusion}

13. Lesotho's risk of debt distress has increased since the analysis done in February 2010 for the 2009 Article IV consultation owing to a new nonconcessional loan for construction of the Metolong dam. The risk of debt distress is magnified in the event of adverse shocks to economic growth, large exchange rate depreciation, or a lower long-run level of SACU revenues. The risks appear manageable over the medium term if the authorities implement the planned fiscal adjustments. Hence, these results underscore the critical need to realign spending with the sustainable level of SACU revenues, while accelerating structural reforms to boost productivity and competitiveness in order to accelerate medium-term growth.

\section{The significant level of gross remittances from migrant workers received by} Lesotho mitigates debt vulnerabilities substantially. When these remittances are taken into account, Lesotho remains at a moderate risk of debt distress. In particular, the PV of external debt to the sum of GDP and gross remittances would peak at 34.4 percent in 2020 (below the modified threshold of 36 percent), compared with a ratio of 43.1 percent excluding these transfers. Thus, using the remittance-modified debt indicators, Lesotho's debt ratios are well below the modified threshold. 
Table 1a. External Debt Sustainability Framework, Baseline Scenario, 2007-2030 1/ (In percent of GDP, unless otherwise indicated)

\begin{tabular}{|c|c|c|c|c|c|c|c|c|c|c|c|c|c|c|c|}
\hline & \multicolumn{3}{|c|}{ Actual } & \multicolumn{2}{|c|}{$\begin{array}{l}\text { Historical } 0 \text { Standard } \\
\text { Average } 0 \text { Deviation }\end{array}$} & \multicolumn{6}{|c|}{ Projections } & & \multirow{2}{*}{$\begin{array}{c}\text { 2016-2030 } \\
\text { Average }\end{array}$} \\
\hline & 2007 & 2008 & 2009 & & & 2010 & 2011 & 2012 & 2013 & 2014 & 2015 & $\begin{array}{c}2010-2015 \\
\text { Average }\end{array}$ & 2020 & 2030 & \\
\hline External debt (nominal) $1 /$ & 50.9 & 47.2 & 36.3 & & & 40.1 & 43.7 & 46.8 & 49.5 & 49.1 & 48.5 & & 44.7 & 16.2 & \\
\hline $\mathrm{o} / \mathrm{w}$ public and publicly guaranteed (PPG) & 50.9 & 47.2 & 36.3 & & & 40.1 & 43.7 & 46.8 & 49.5 & 49.1 & 48.5 & & 44.7 & 16.2 & \\
\hline Change in external debt & 2.0 & -3.7 & -10.9 & & & 3.8 & 3.6 & 3.1 & 2.7 & -0.4 & -0.6 & & -3.2 & -2.9 & \\
\hline Identified net debt-creating flows & -25.6 & -15.9 & -4.4 & & & 17.6 & 18.8 & 14.5 & 5.4 & 1.0 & 0.2 & & -5.3 & -5.4 & \\
\hline Non-interest current account deficit & -18.1 & -9.8 & 0.9 & 5.0 & 13.1 & 22.6 & 23.2 & 19.4 & 9.2 & 5.6 & 7.9 & & -1.2 & -1.8 & 0.5 \\
\hline Deficit in balance of goods and services & 52.8 & 53.9 & 59.9 & & & 63.3 & 58.9 & 59.6 & 50.8 & 49.5 & 52.3 & & 42.2 & 41.3 & \\
\hline Exports & 55.8 & 58.6 & 49.7 & & & 47.4 & 47.9 & 46.8 & 48.9 & 46.8 & 43.6 & & 45.8 & 47.8 & \\
\hline Imports & 108.6 & 112.5 & 109.6 & & & 110.7 & 106.9 & 106.4 & 99.7 & 96.3 & 95.9 & & 88.0 & 89.1 & \\
\hline Net current transfers (negative $=$ inflow) & -39.8 & -32.0 & -32.1 & -24.9 & 7.6 & -17.1 & -11.3 & -16.9 & -18.0 & -17.9 & -17.8 & & -17.7 & -17.4 & -17.6 \\
\hline $\mathrm{o} / \mathrm{w}$ official & -23.6 & -39.3 & -31.4 & & & -28.9 & -16.4 & -10.6 & -16.1 & -17.1 & -16.1 & & -17.1 & -16.8 & \\
\hline Other current account flows (negative $=$ net inflow) & -31.1 & -31.7 & -26.9 & & & -23.5 & -24.4 & -23.3 & -23.6 & -26.0 & -26.6 & & -25.7 & -25.6 & \\
\hline Net FDI (negative = inflow) & -6.7 & -5.8 & -5.0 & -5.0 & 1.0 & -3.6 & -3.6 & -3.4 & -3.4 & -3.4 & -3.4 & & -3.4 & -3.4 & -3.4 \\
\hline Endogenous debt dynamics 2 / & -0.8 & -0.3 & -0.3 & & & -1.5 & -0.8 & -1.5 & -0.4 & -1.3 & -4.3 & & -0.7 & -0.2 & \\
\hline Contribution from nominal interest rate & 4.2 & 0.3 & 0.5 & & & 0.4 & 0.4 & 0.7 & 0.9 & 1.1 & 1.1 & & 1.6 & 0.7 & \\
\hline Contribution from real GDP growth & -1.1 & -2.2 & -0.4 & & & -1.8 & -1.3 & -2.2 & -1.3 & -2.4 & -5.4 & & -2.3 & -0.9 & \\
\hline Contribution from price and exchange rate changes & -3.9 & 1.6 & -0.4 & & & $\ldots$ & $\ldots$ & $\ldots$ & $\ldots$ & $\ldots$ & $\ldots$ & & $\ldots$ & $\ldots$ & \\
\hline Residual (3-4) 3/ & 27.6 & 12.2 & -6.5 & & & -13.8 & -15.2 & -11.4 & -2.7 & -1.4 & -0.7 & & 2.0 & 2.5 & \\
\hline $\mathrm{o} / \mathrm{w}$ exceptional financing & 0.0 & 0.0 & 0.0 & & & 0.0 & 0.0 & 0.0 & 0.0 & 0.0 & 0.0 & & 0.0 & 0.0 & \\
\hline PV of external debt 4/ & $\ldots$ & $\ldots$ & 25.5 & & & 28.3 & 33.0 & 37.4 & 41.3 & 41.3 & 42.2 & & 43.1 & 15.3 & \\
\hline PV of PPG external debt & ... & ... & 25.5 & & & 28.3 & 33.0 & 37.4 & 41.3 & 41.3 & 42.2 & & 43.1 & 15.3 & \\
\hline In percent of exports & ... & ... & 51.2 & & & 59.6 & 68.9 & 80.0 & 84.5 & 88.2 & 96.9 & & 94.0 & 31.9 & \\
\hline In percent of government revenues & $\ldots$ & $\ldots$ & 40.2 & & & 62.0 & 84.0 & 82.0 & 87.9 & 87.3 & 91.6 & & 93.4 & 33.1 & \\
\hline Debt service-to-exports ratio (in percent) & 10.6 & 2.7 & 4.2 & & & 4.2 & 4.2 & 4.5 & 4.6 & 4.8 & 5.2 & & 7.3 & 6.3 & \\
\hline PPG debt service-to-exports ratio (in percent) & 10.6 & 2.7 & 4.2 & & & 4.2 & 4.2 & 4.5 & 4.6 & 4.8 & 5.2 & & 7.3 & 6.3 & \\
\hline PPG debt service-to-revenue ratio (in percent) & 9.5 & 2.5 & 3.3 & & & 4.4 & 5.1 & 4.6 & 4.8 & 4.8 & 4.9 & & 7.2 & 6.6 & \\
\hline Total gross financing need (Millions of U.S. dollars) & -298.1 & -224.5 & -33.1 & & & 378.8 & 404.4 & 357.6 & 166.7 & 96.8 & 160.8 & & -34.3 & -82.2 & \\
\hline Non-interest current account deficit that stabilizes debt ratio & -20.1 & -6.1 & 11.8 & & & 18.8 & 19.6 & 16.3 & 6.5 & 6.1 & 8.4 & & 2.1 & 1.2 & \\
\hline \multicolumn{16}{|l|}{ Key macroeconomic assumptions } \\
\hline Real GDP growth (in percent) & 2.4 & 4.5 & 0.9 & 3.3 & 2.0 & 5.6 & 3.3 & 5.3 & 3.0 & 5.0 & 12.1 & 5.7 & 5.0 & 5.0 & 5.0 \\
\hline GDP deflator in US dollar terms (change in percent) & 8.7 & -3.1 & 0.9 & 5.3 & 16.7 & 4.9 & 0.4 & 0.5 & 1.6 & -0.4 & -1.3 & 1.0 & -1.8 & -1.8 & -1.8 \\
\hline Effective interest rate (percent) $5 /$ & 9.6 & 0.6 & 1.1 & 2.9 & 3.7 & 1.1 & 1.1 & 1.6 & 2.0 & 2.3 & 2.4 & 1.8 & 3.5 & 3.9 & 3.5 \\
\hline Growth of exports of G\&S (US dollar terms, in percent) & 16.0 & 6.3 & -13.7 & 15.5 & 18.8 & 5.6 & 5.0 & 3.3 & 9.3 & 0.3 & 2.9 & 4.4 & 3.7 & 3.4 & 3.7 \\
\hline Growth of imports of G\&S (US dollar terms, in percent) & 17.2 & 4.8 & -0.9 & 8.7 & 13.8 & 11.9 & 0.2 & 5.3 & -2.0 & 1.1 & 10.1 & 4.4 & -1.6 & 3.2 & 2.6 \\
\hline Grant element of new public sector borrowing (in percent) & & $\ldots$ & $\ldots$ & $\ldots$ & ... & 31.6 & -0.2 & -2.0 & -3.8 & 14.6 & -5.3 & 5.8 & 291.8 & 121.9 & 44.6 \\
\hline Government revenues (excluding grants, in percent of GDP) & 61.9 & 62.3 & 63.4 & & & 45.6 & 39.3 & 45.6 & 47.0 & 47.3 & 46.1 & & 46.1 & 46.1 & 46.1 \\
\hline Aid flows (in Millions of US dollars) $7 /$ & 22.3 & 25.4 & 61.9 & & & 123.0 & 189.4 & 173.6 & 123.9 & 86.0 & 82.7 & & 96.3 & 130.6 & \\
\hline $\mathrm{o} / \mathrm{w}$ Grants & 22.3 & 25.4 & 61.9 & & & 123.0 & 189.4 & 173.6 & 123.9 & 86.0 & 82.7 & & 96.3 & 130.6 & \\
\hline $\mathrm{o} / \mathrm{w}$ Concessional loans & 0.0 & 0.0 & 0.0 & & & 0.0 & 0.0 & 0.0 & 0.0 & 0.0 & 0.0 & & 0.0 & 0.0 & \\
\hline Grant-equivalent financing (in percent of GDP) $8 /$ & $\ldots$ & $\ldots$ & $\ldots$ & & & 7.8 & 10.1 & 8.6 & 5.8 & 4.4 & 3.2 & & 3.5 & 3.5 & 3.3 \\
\hline Grant-equivalent financing (in percent of external financing) 8/ & $\ldots$ & $\ldots$ & $\ldots$ & & & 79.5 & 60.0 & 55.4 & 48.0 & 64.6 & 36.2 & & 100.0 & 100.0 & 85.6 \\
\hline PV of PPG external debt (in Millions of US dollars) & & & 474.6 & & & 493.9 & 601.9 & 722.1 & 833.9 & 870.7 & 986.0 & & 1171.0 & 562.3 & \\
\hline (PVt-PVt-1)/GDPt-1 (in percent) & & & & & & 1.2 & 6.0 & 6.4 & 5.7 & 1.8 & 5.3 & 4.4 & -1.6 & -2.4 & -0.7 \\
\hline Gross remittances (Millions of US dollars) & 437.4 & 426.0 & 381.4 & & & 383.2 & 428.6 & 441.8 & 463.8 & 530.1 & 605.3 & & 696.2 & 925.3 & \\
\hline PV of PPG external debt (in percent of GDP + remittances) & $\ldots$ & $\ldots$ & 20.6 & & & 23.3 & 26.9 & 30.6 & 33.7 & 33.2 & 33.7 & & 34.4 & 12.3 & \\
\hline PV of PPG external debt (in percent of exports + remittances) & $\ldots$ & $\ldots$ & 34.8 & & & 41.1 & 46.6 & 54.1 & 57.9 & 57.9 & 61.3 & & 60.8 & 21.1 & \\
\hline Debt service of PPG external debt (in percent of exports + remittances) & ... & $\ldots$ & 2.9 & & & 2.9 & 2.8 & 3.0 & 3.2 & 3.2 & 3.3 & & 4.7 & 4.2 & \\
\hline
\end{tabular}

Sources: Country authorities; and staff estimates and projections.

$1 /$ Includes both public and private sector external debt.

2/ Derived as $[\mathrm{r}-\mathrm{g}-\rho(1+\mathrm{g})](1+\mathrm{g}+\rho+\mathrm{g} \rho)$ times previous period debt ratio, with $\mathrm{r}=$ nominal interest rate; $\mathrm{g}=$ real GDP growth rate, and $\rho=$ growth rate of GDP deflator in U.S. dollar terms.

3/ Includes exceptional financing (i.e., changes in arrears and debt relief); changes in gross foreign assets; and valuation adjustments. For projections also includes contribution from price and exchange rate changes.

4/ Assumes that PV of private sector debt is equivalent to its face value.

$5 /$ Current-year interest payments divided by previous period debt stock.

6/ Historical averages and standard deviations are generally derived over the past 10 years, subject to data availability.

7/ Defined as grants, concessional loans, and debt relief.

8/ Grant-equivalent financing includes grants provided directly to the government and through new borrowing (difference between the face value and the PV of new debt). 
Table 1b. Lesotho: Sensitivity Analysis for Key Indicators of Public and Publicly Guaranteed

External Debt, 2010-2030

(In percent)

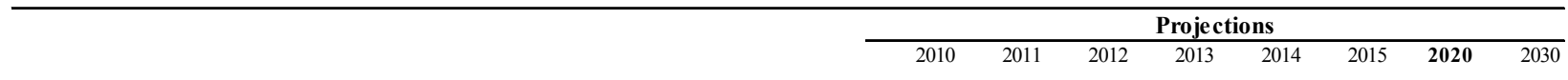

PV of debt-to GDP ratio

Baseline

A. Alternative Scenarios

A1. Key variables at their historical averages in 2010-2030 1/

A2. New public sector loans on less favorable terms in 2010-20302

A3. Alternative Scenario :No LHWP2

B. Bound Tests

B1. Real GDP growth at historical average minus one standard deviation in 2011-2012 B2. Export value growth at historical average minus one standard deviation in 2011-2012 3/ B3. US dollar GDP deflator at historical average minus one standard deviation in 2011-2012 B4. Net non-debt creating flows at historical average minus one standard deviation in 2011-2012 4/ B5. Combination of B1-B4 using one-half standard deviation shocks

B6. One-time 30 percent nominal depreciation relative to the baseline in $20115 /$
28

$28 \quad 15$

28

\section{PV of debt-to-exports ratio}

Baseline

\section{A. Alternative Scenarios}

A1. Key variables at their historical averages in 2010-2030 1/

A2. New public sector loans on less favorable terms in 2010-2030 2

A3. Alternative Scenario :No LHWP2

B. Bound Tests

B1. Real GDP growth at historical average minus one standard deviation in 2011-2012 B2. Export value growth at historical average minus one standard deviation in 2011-2012 3/ B3. US dollar GDP deflator at historical average minus one standard deviation in 2011-2012 B4. Net non-debt creating flows at historical average minus one standard deviation in 2011-2012 4/ B5. Combination of B1-B4 using one-half standard deviation shocks

B6. One-time 30 percent nominal depreciation relative to the baseline in $20115 /$

69

\section{9}

80

$\begin{array}{lll}60 & 32 & 15\end{array}$

$60 \quad 66$

79

$\begin{array}{rrrrr}12 & 9 & 9 & \mathbf{0} & 29 \\ 85 & 91 & 100 & \mathbf{1 0 2} & 40 \\ 81 & 82 & 80 & 51 & 11\end{array}$

PV of debt-to-revenue ratio

Baseline

A. Alternative Scenarios

A1. Key variables at their historical averages in 2010-2030 1/

A2. New public sector loans on less favorable terms in 2010-2030 2

A3. Alternative Scenario :No LHWP2

$\begin{array}{llllrrrr}62 & 39 & 15 & 13 & 9 & 9 & \mathbf{0} & 30 \\ 62 & 80 & 81 & 89 & 90 & 95 & \mathbf{1 0 2} & 41 \\ 62 & 84 & 80 & 85 & 81 & 76 & 51 & 12\end{array}$

B. Bound Tests

B1. Real GDP growth at historical average minus one standard deviation in 2011-2012 B2. Export value growth at historical average minus one standard deviation in 2011-2012 3/

B3. US dollar GDP deflator at historical average minus one standard deviation in 2011-2012

B4. Net non-debt creating flows at historical average minus one standard deviation in 2011-2012 4/

B5. Combination of B1-B4 using one-half standard deviation shocks

B6. One-time 30 percent nominal depreciation relative to the baseline in 2011 5/

$\begin{array}{rrrrrrrr}62 & 84 & 85 & 91 & 90 & 95 & \mathbf{9 6} & 33 \\ 62 & 90 & 98 & 103 & 102 & 105 & \mathbf{1 0 7} & 44 \\ 62 & 93 & 103 & 111 & 110 & 115 & \mathbf{1 1 7} & 40 \\ 62 & 69 & 67 & 74 & 73 & 78 & \mathbf{7 9} & 23 \\ 62 & 65 & 62 & 69 & 69 & 75 & \mathbf{7 6} & 16 \\ 62 & 117 & 114 & 122 & 121 & 127 & \mathbf{1 2 9} & 45\end{array}$


Table 1b. Lesotho: Sensitivity Analysis for Key Indicators of Public and Publicly Guaranteed

External Debt, 2010-2030 (concluded)

(In percent)

\section{Debt service-to-exports ratio}

Baseline

A. Alternative Scenarios

A1. Key variables at their historical averages in 2010-2030 1/

A2. New public sector loans on less favorable terms in 2010-2030 2

A3. Alternative Scenario :No LHWP2

B. Bound Tests

B1. Real GDP growth at historical average minus one standard deviation in 2011-2012

B2. Export value growth at historical average minus one standard deviation in 2011-2012 3/

B3. US dollar GDP deflator at historical average minus one standard deviation in 2011-2012

B4. Net non-debt creating flows at historical average minus one standard deviation in 2011-2012 4/

B5. Combination of B1-B4 using one-half standard deviation shocks

B6. One-time 30 percent nominal depreciation relative to the baseline in 20115 /

$\begin{array}{ll}4 & 4 \\ & \\ 4 & 4 \\ 4 & 4 \\ 4 & 4\end{array}$

$\begin{array}{llllllll}4 & 4 & 5 & 5 & 5 & 5 & 7 & 6 \\ 4 & 5 & 5 & 6 & 6 & 7 & 9 & 8 \\ 4 & 4 & 5 & 5 & 5 & 5 & 7 & 6 \\ 4 & 4 & 4 & 4 & 4 & 5 & 7 & 6 \\ 4 & 4 & 4 & 4 & 4 & 4 & 6 & 5 \\ 4 & 4 & 5 & 5 & 5 & 5 & 7 & 6\end{array}$

\section{Debt service-to-revenue ratio}

Baseline

4

A. Alternative Scenarios

A1. Key variables at their historical averages in 2010-2030 1/

A2. New public sector loans on less favorable terms in 2010-2030 2

A3. Alternative Scenario :No LHWP2

$\begin{array}{llllllll}4 & 5 & 3 & 3 & 2 & 2 & \mathbf{3} & 2 \\ 4 & 5 & 4 & 5 & 5 & 5 & 9 & 6 \\ 4 & 5 & 5 & 5 & 5 & 5 & 5 & 3\end{array}$

B. Bound Tests

B1. Real GDP growth at historical average minus one standard deviation in 2011-2012

B2. Export value growth at historical average minus one standard deviation in 2011-2012 3/

B3. US dollar GDP deflator at historical average minus one standard deviation in 2011-2012

B4. Net non-debt creating flows at historical average minus one standard deviation in 2011-2012 4/

B5. Combination of B1-B4 using one-half standard deviation shocks

B6. One-time 30 percent nominal depreciation relative to the baseline in 2011 5/

Memorandum item:

Grant element assumed on residual financing (i.e., financing required above baseline) $6 /$

$\begin{array}{rrrrrrrr}4 & 5 & 5 & 5 & 5 & 5 & \mathbf{8} & 7 \\ 4 & 5 & 5 & 5 & 5 & 5 & \mathbf{8} & 7 \\ 4 & 6 & 6 & 6 & 6 & 6 & \mathbf{9} & 8 \\ 4 & 5 & 4 & 4 & 4 & 5 & 7 & 6 \\ 4 & 5 & 5 & 5 & 5 & 5 & \mathbf{7} & 6 \\ 4 & 7 & 7 & 7 & 7 & 7 & \mathbf{1 0} & 9 \\ & & & & & & & \\ 21 & 21 & 21 & 21 & 21 & 21 & \mathbf{2 1} & 21\end{array}$

Sources: Country authorities; and staff estimates and projections.

1/ Variables include real GDP growth, growth of GDP deflator (in U.S. dollar terms), non-interest current account in percent of GDP, and non-debt creating flows.

2/ Assumes that the interest rate on new borrowing is by 2 percentage points higher than in the baseline., while grace and maturity periods are the same as in the baseline.

3/ Exports values are assumed to remain permanently at the lower level, but the current account as a share of GDP is assumed to return to its baseline level after the shock

(implicitly assuming an offsetting adjustment in import levels).

4/ Includes official and private transfers and FDI.

5/ Depreciation is defined as percentage decline in dollar/local currency rate, such that it never exceeds 100 percent.

6/ Applies to all stress scenarios except for A2 (less favorable financing) in which the terms on all new financing are as specified in footnote 2. 
Table 2a. Lesotho: Public Sector Debt Sustainability Framework, Baseline Scenario, 2007-2030

(In percent of GDP, unless otherwise indicated)

\begin{tabular}{|c|c|c|c|c|c|c|c|c|c|c|c|c|c|c|c|}
\hline & \multicolumn{3}{|c|}{ Actual } & \multirow[b]{2}{*}{ Average } & \multirow[b]{2}{*}{$\begin{array}{c}\text { Standard } \\
\text { Deviation } \\
\end{array}$} & \multicolumn{4}{|l|}{ Estimate } & \multicolumn{3}{|c|}{ Projections } & \multirow[b]{2}{*}{2020} & \multirow[b]{2}{*}{2030} & \multirow[b]{2}{*}{$\begin{array}{l}2016-30 \\
\text { Average }\end{array}$} \\
\hline & 2007 & 2008 & 2009 & & & 2010 & 2011 & 2012 & 2013 & 2014 & 2015 & $\begin{array}{l}2010-15 \\
\text { Average }\end{array}$ & & & \\
\hline $\begin{array}{l}\text { Public sector debt 1/ } \\
\text { o/w foreign-currency denominated }\end{array}$ & $\begin{array}{l}56.7 \\
50.9\end{array}$ & $\begin{array}{l}52.1 \\
47.2\end{array}$ & $\begin{array}{l}40.2 \\
36.3\end{array}$ & & & $\begin{array}{l}45.2 \\
40.1\end{array}$ & $\begin{array}{l}49.8 \\
43.7\end{array}$ & $\begin{array}{l}53.1 \\
46.8\end{array}$ & $\begin{array}{l}55.3 \\
49.5\end{array}$ & $\begin{array}{l}54.4 \\
49.1\end{array}$ & $\begin{array}{l}52.4 \\
48.5\end{array}$ & & $\begin{array}{l}51.3 \\
44.7\end{array}$ & $\begin{array}{l}24.4 \\
16.2\end{array}$ & \\
\hline Change in public sector debt & 0.5 & -4.6 & -11.9 & & & 5.0 & 4.6 & 3.3 & 2.2 & -0.9 & -1.9 & & -2.9 & -2.9 & \\
\hline Identified debt-creating flows & -19.3 & 3.7 & -10.1 & & & 17.2 & 16.2 & 6.3 & 0.8 & -1.4 & -6.3 & & -2.5 & -1.8 & \\
\hline Primary deficit & -14.6 & -3.5 & 1.1 & -3.4 & 7.0 & 14.5 & 17.6 & 8.4 & 2.1 & -0.3 & -2.2 & 6.7 & -2.6 & -1.9 & -2.3 \\
\hline Revenue and grants & 63.3 & 63.9 & 67.2 & & & 52.4 & 49.5 & 54.4 & 53.0 & 51.3 & 49.6 & & 49.6 & 49.6 & \\
\hline of which: grants & 1.4 & 1.6 & 3.8 & & & 6.8 & 10.1 & 8.8 & 6.0 & 4.0 & 3.5 & & 3.5 & 3.5 & \\
\hline Primary (noninterest) expenditure & 48.8 & 60.4 & 68.2 & & & 66.9 & 67.1 & 62.8 & 55.1 & 51.0 & 47.3 & & 47.0 & 47.7 & \\
\hline Automatic debt dynamics & -4.7 & 7.2 & -11.1 & & & 2.8 & -1.4 & -2.0 & -1.3 & -1.1 & -4.1 & & 0.1 & 0.1 & \\
\hline Contribution from interest rate/growth differential & -3.7 & -8.6 & -3.3 & & & -3.7 & -3.3 & -4.1 & -2.8 & -3.6 & -6.8 & & -3.0 & -1.2 & \\
\hline of which: contribution from average real interest rate & -2.4 & -6.2 & -2.8 & & & -1.5 & -1.8 & -1.6 & -1.3 & -1.0 & -0.9 & & -0.4 & 0.1 & \\
\hline of which: contribution from real GDP growth & -1.3 & -2.4 & -0.4 & & & -2.1 & -1.4 & -2.5 & -1.5 & -2.6 & -5.9 & & -2.6 & -1.3 & \\
\hline Contribution from real exchange rate depreciation & -1.0 & 15.8 & -7.9 & & & 6.4 & 1.9 & 2.1 & 1.5 & 2.6 & 2.7 & & $\ldots$ & $\ldots$ & \\
\hline Other identified debt-creating flows & 0.0 & 0.0 & 0.0 & & & 0.0 & 0.0 & 0.0 & 0.0 & 0.0 & 0.0 & & 0.0 & 0.0 & \\
\hline Privatization receipts (negative) & 0.0 & 0.0 & 0.0 & & & 0.0 & 0.0 & 0.0 & 0.0 & 0.0 & 0.0 & & 0.0 & 0.0 & \\
\hline Recognition of implicit or contingent liabilities & 0.0 & 0.0 & 0.0 & & & 0.0 & 0.0 & 0.0 & 0.0 & 0.0 & 0.0 & & 0.0 & 0.0 & \\
\hline Debt relief (HIPC and other) & 0.0 & 0.0 & 0.0 & & & 0.0 & 0.0 & 0.0 & 0.0 & 0.0 & 0.0 & & 0.0 & 0.0 & \\
\hline Other (specify, e.g. bank recapitalization) & 0.0 & 0.0 & 0.0 & & & 0.0 & 0.0 & 0.0 & 0.0 & 0.0 & 0.0 & & 0.0 & 0.0 & \\
\hline Residual, including asset changes & 19.8 & -8.3 & -1.9 & & & -12.2 & -11.5 & -3.0 & 1.3 & 0.5 & 4.4 & & -0.4 & -1.2 & \\
\hline \multicolumn{16}{|l|}{ Other S ustainability Indicators } \\
\hline PV of public sector debt & 5.8 & 4.9 & 29.3 & & & 33.3 & 39.1 & 43.8 & 47.1 & 46.6 & 46.2 & & 49.7 & 23.5 & \\
\hline $\mathrm{o} / \mathrm{w}$ foreign-currency denominated & 0.0 & 0.0 & 25.5 & & & 28.3 & 33.0 & 37.4 & 41.3 & 41.3 & 42.2 & & 43.1 & 15.3 & \\
\hline $\mathrm{o} / \mathrm{w}$ external & $\ldots$ & $\ldots$ & 25.5 & & & 28.3 & 33.0 & 37.4 & 41.3 & 41.3 & 42.2 & & 43.1 & 15.3 & \\
\hline $\mathrm{PV}$ of contingent liabilities (not included in public sector debt) & $\ldots$ & $\ldots$ & & & & $\ldots$ & ... & ... & $\ldots$ & $\ldots$ & $\ldots$ & & $\ldots$ & $\ldots$ & \\
\hline Gross financing need $2 /$ & -8.7 & -1.9 & 3.2 & & & 16.8 & 19.8 & 10.8 & 4.8 & 2.4 & 0.4 & & 1.2 & 1.7 & \\
\hline $\mathrm{PV}$ of public sector debt-to-revenue and grants ratio (in percent) & 9.2 & 7.7 & 43.7 & & & 63.6 & 79.1 & 80.5 & 89.0 & 90.9 & 93.1 & & 100.3 & 47.4 & \\
\hline $\mathrm{PV}$ of public sector debt-to-revenue ratio (in percent) & 9.4 & 7.9 & 46.3 & & & 73.1 & 99.5 & 95.9 & 100.3 & 98.6 & 100.1 & & 107.8 & 51.0 & \\
\hline o/w external $3 /$ & & $\ldots$ & 40.2 & & & 62.0 & 84.0 & 82.0 & 87.9 & 87.3 & 91.6 & & 93.4 & 33.1 & \\
\hline Debt service-to-revenue and grants ratio (in percent) 4/ & 9.3 & 2.5 & 3.1 & & & 4.5 & 4.6 & 4.5 & 5.0 & 5.2 & 5.3 & & 7.5 & 7.2 & \\
\hline Debt service-to-revenue ratio (in percent) $4 /$ & 9.5 & 2.5 & 3.3 & & & 5.1 & 5.8 & 5.3 & 5.6 & 5.7 & 5.7 & & 8.1 & 7.8 & \\
\hline Primary deficit that stabilizes the debt-to-GDP ratio & -15.1 & 1.2 & 13.0 & & & 9.4 & 12.9 & 5.0 & 0.0 & 0.6 & -0.3 & & 0.3 & 1.1 & \\
\hline \multicolumn{16}{|l|}{ Key macroeconomic and fiscal assumptions } \\
\hline Real GDP growth (in percent) & 2.4 & 4.5 & 0.9 & 3.3 & 2.0 & 5.6 & 3.3 & 5.3 & 3.0 & 5.0 & 12.1 & 5.7 & 5.0 & 5.0 & 5.0 \\
\hline Average nominal interest rate on forex debt (in percent) & 9.6 & 0.6 & 1.1 & 2.9 & 3.7 & 1.1 & 1.1 & 1.6 & 2.0 & 2.3 & 2.4 & 1.8 & 3.5 & 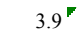 & 3.5 \\
\hline Average real interest rate on domestic debt (in percent) & $\ldots$ & & & $\ldots$ & $\ldots$ & 3.5 & -0.8 & 0.7 & 0.5 & 3.5 & $4.2^{r}$ & 1.9 & 3.8 & $4.2^{\mathrm{F}}$ & 4.1 \\
\hline Real exchange rate depreciation (in percent,+ indicates depreciation) & -2.1 & 36.5 & -17.8 & 6.4 & 29.3 & 19.7 & $\ldots$ & $\ldots$ & $\ldots$ & $\ldots$ & $\ldots$ & $\ldots$ & $\ldots$ & $\ldots$ & $\ldots$ \\
\hline Inflation rate (GDP deflator, in percent) & 13.1 & 13.6 & 3.4 & 7.7 & 4.5 & 6.0 & 6.3 & 5.4 & 6.4 & 4.3 & 3.7 & 5.3 & 3.0 & 3.0 & 3.0 \\
\hline Growth of real primary spending (deflated by GDP deflator, in percent) & 0.0 & 0.3 & 0.1 & 0.0 & 0.1 & 0.0 & 0.0 & 0.0 & -0.1 & 0.0 & 0.0 & 0.0 & 0.0 & 0.1 & 0.1 \\
\hline Grant element of new external borrowing (in percent) & $\ldots$ & $\ldots$ & $\ldots$ & $\ldots$ & $\ldots$ & 31.6 & -0.2 & -2.0 & -3.8 & 14.6 & -5.3 & 5.8 & 291.8 & 121.9 & \\
\hline
\end{tabular}

Sources: Country authorities; and staff estimates and projections.

[Indicate coverage of public sector, e.g., general government or nonfinancial public sector. Also whether net or gross debt is used.]

2/ Gross financing need is defined as the primary deficit plus debt service plus the stock of short-term debt at the end of the last period.

$3 /$ Revenues excluding grants.

4/ Debt service is defined as the sum of interest and amortization of medium and long-term debt.

$5 /$ Historical averages and standard deviations are generally derived over the past 10 years, subject to data availability. 
Table 2b. Lesotho: Sensitivity Analysis for Key Indicators of Public Debt 2010-2030

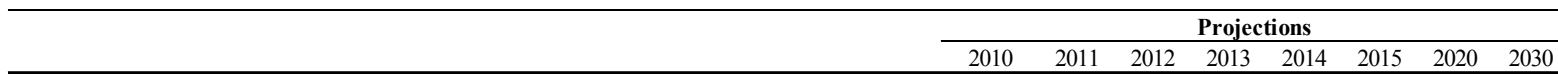

PV of Debt-to-GDP Ratio

Baseline

A. Alternative scenarios

A1. Real GDP growth and primary balance are at historical averages

A2. Primary balance is unchanged from 2010

A3. Permanently lower GDP growth 1/

A4. Alternative Scenario : Permanent decline in SACU revenue

\section{B. Bound tests}

B1. Real GDP growth is at historical average minus one standard deviations in 2011-2012

B2. Primary balance is at historical average minus one standard deviations in 2011-2012

B3. Combination of B1-B2 using one half standard deviation shocks

B5. 10 percent of GDP increase in other debt-creating flows in 2011

PV of Debt-to-Revenue Ratio 2/

Baseline

64

A. Alternative scenarios

A1. Real GDP growth and primary balance are at historical averages

A2. Primary balance is unchanged from 2010

A3. Permanently lower GDP growth $1 /$

A4. Alternative Scenario : Permanent decline in SACU revenue

\section{B. Bound tests}

B1. Real GDP growth is at historical average minus one standard deviations in 2011-2012

B2. Primary balance is at historical average minus one standard deviations in 2011-2012

B3. Combination of B1-B2 using one half standard deviation shocks

B4. One-time 30 percent real depreciation in 2011

B5. 10 percent of GDP increase in other debt-creating flows in 2011
B4. One-time 30 percent real depreciation in 2011

$\begin{array}{llllllll}33 & 39 & 44 & 47 & 47 & 46 & 50 & 23\end{array}$

$\begin{array}{rrrrrrrr}33 & 18 & 12 & 10 & 6 & 8 & 8 & -39 \\ 33 & 36 & 47 & 62 & 77 & 91 & 183 & 345 \\ 33 & 39 & 45 & 49 & 49 & 50 & 63 & 71 \\ 33 & 39 & 44 & 48 & 47 & 47 & 54 & 30\end{array}$

$\begin{array}{rrrrrrrr}33 & 41 & 50 & 56 & 59 & 61 & 79 & 84 \\ 33 & 25 & 25 & 29 & 28 & 29 & 32 & 4 \\ 33 & 22 & 19 & 25 & 26 & 29 & 41 & 32 \\ 33 & 52 & 57 & 61 & 61 & 60 & 70 & 53 \\ 33 & 49 & 54 & 57 & 56 & 55 & 59 & 34\end{array}$

$\begin{array}{llllllll}64 & 79 & 80 & 89 & 91 & 93 & 100 & 47\end{array}$

$\begin{array}{llllllll}64 & 37 & 22 & 18 & 12 & 17 & 15 & -77\end{array}$

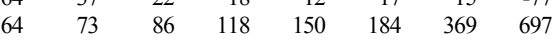

$\begin{array}{llllllll}64 & 80 & 82 & 92 & 96 & 101 & 127 & 143\end{array}$

$\begin{array}{llllllll}64 & 79 & 81 & 90 & 93 & 95 & 109 & 131\end{array}$

$\begin{array}{rrrrrrrr}64 & 82 & 91 & 106 & 114 & 122 & 160 & 169 \\ 64 & 51 & 47 & 54 & 55 & 58 & 64 & 7 \\ 64 & 44 & 35 & 46 & 51 & 58 & 82 & 65 \\ 64 & 106 & 105 & 115 & 119 & 122 & 142 & 107 \\ 64 & 99 & 99 & 107 & 110 & 112 & 120 & 69\end{array}$

Baseline

Debt Service-to-Revenue Ratio 2/

\section{A. Alternative scenarios}

A1. Real GDP growth and primary balance are at historical averages

A2. Primary balance is unchanged from 2010

A3. Permanently lower GDP growth 1/

A4. Alternative Scenario : Permanent decline in SACU revenue

\section{B. Bound tests}

B1. Real GDP growth is at historical average minus one standard deviations in 2011-2012

B2. Primary balance is at historical average minus one standard deviations in 2011-2012

B3. Combination of B1-B2 using one half standard deviation shocks

B4. One-time 30 percent real depreciation in 2011

B5. 10 percent of GDP increase in other debt-creating flows in 2011 
Figure 1. Lesotho: Indicators of Public and Publicly Guaranteed External Debt under Alternatives Scenarios, 2010-2030 1/
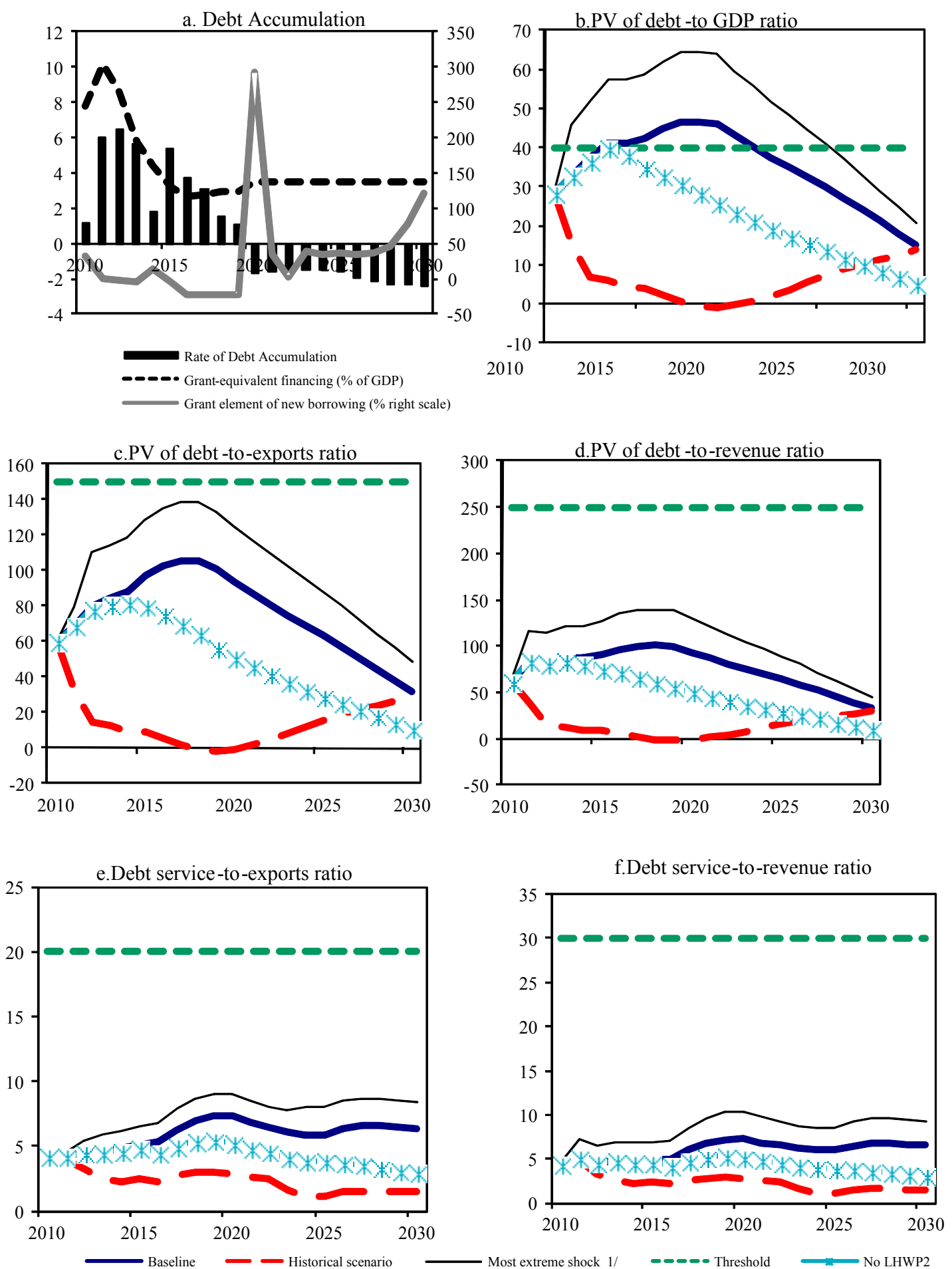

Sources: Country authorities; and staff estimates and projections.

1/ The most extreme stress test is the test that yields the highest ratio in 2020. In figure b. it corresponds to a One-time depreciation shock; in c. to a Exports shock; in d. to a One-time depreciation shock; in e. to a Exports shock and in figure f. to a One-time depreciation shock 
Figure 2. Lesotho: Indicators of Public Debt Under Alternative Scenarios, 2010-2030 1/
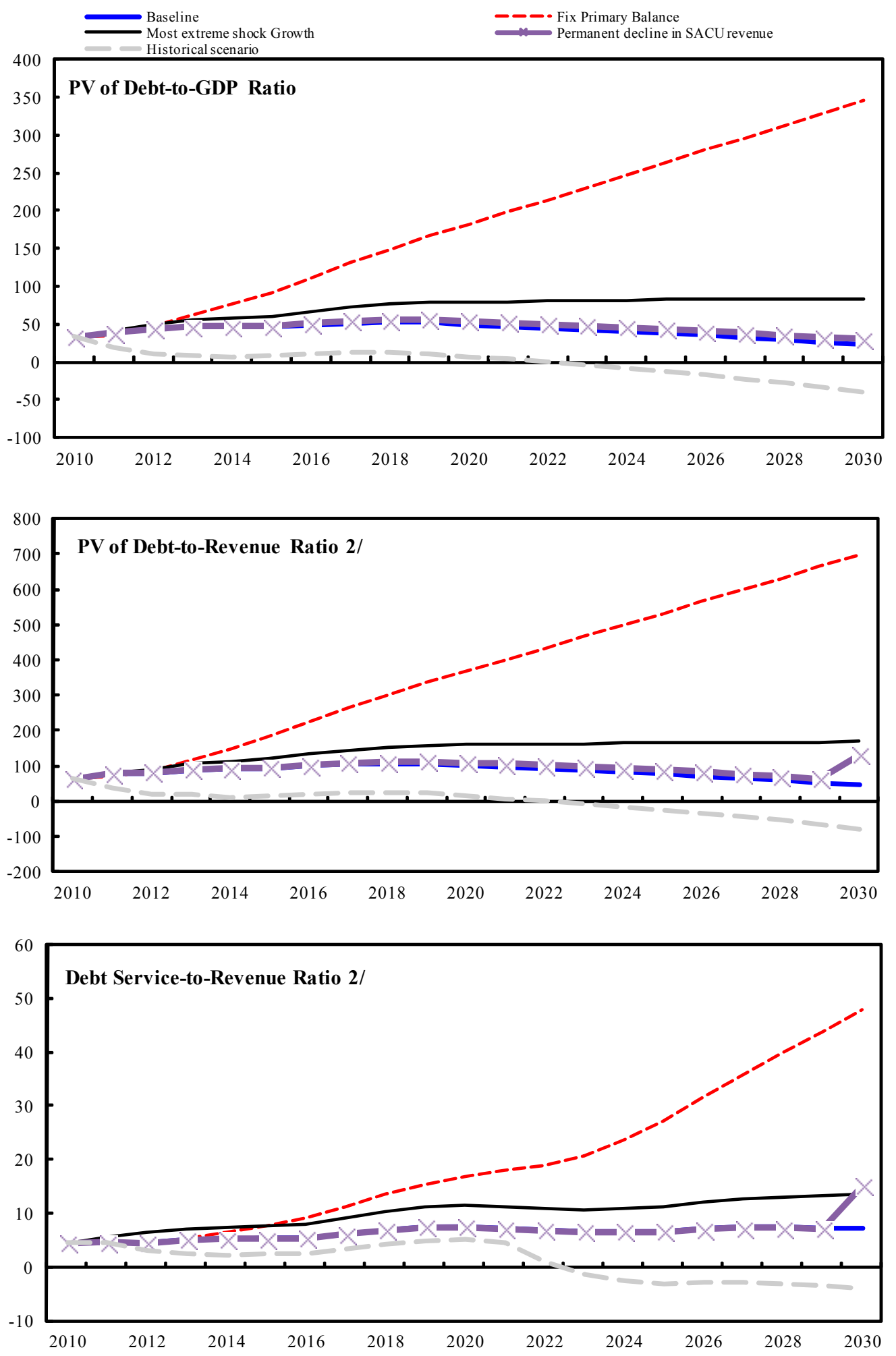

Sources: Country authorities; and staff estimates and projections.

1/ The most extreme stress test is the test that yields the highest ratio in 2020.

2/ Revenues are defined inclusive of grants. 
Figure 3. Lesotho: Indicators of Public and Publicly Guaranteed External Debt under Alternatives Scenarios, 2010-2030 1/

a. Debt Accumulation

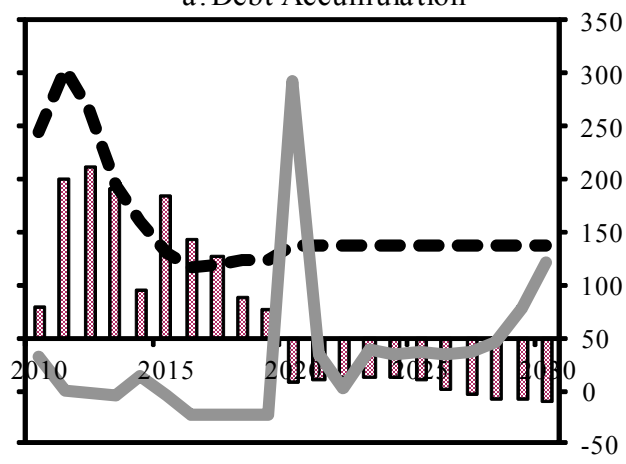

Rate of Debt Accumulation

- Grant-equivalent financing (\% of GDP)

Grant element of new borrowing (\% right scale)
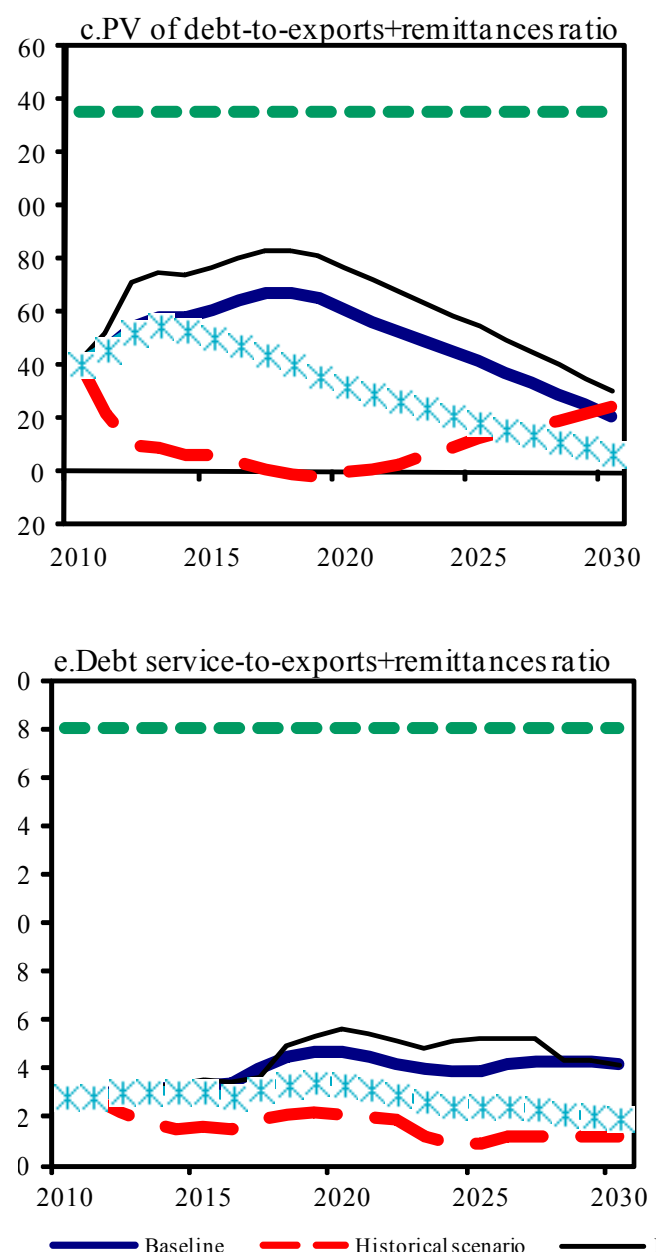
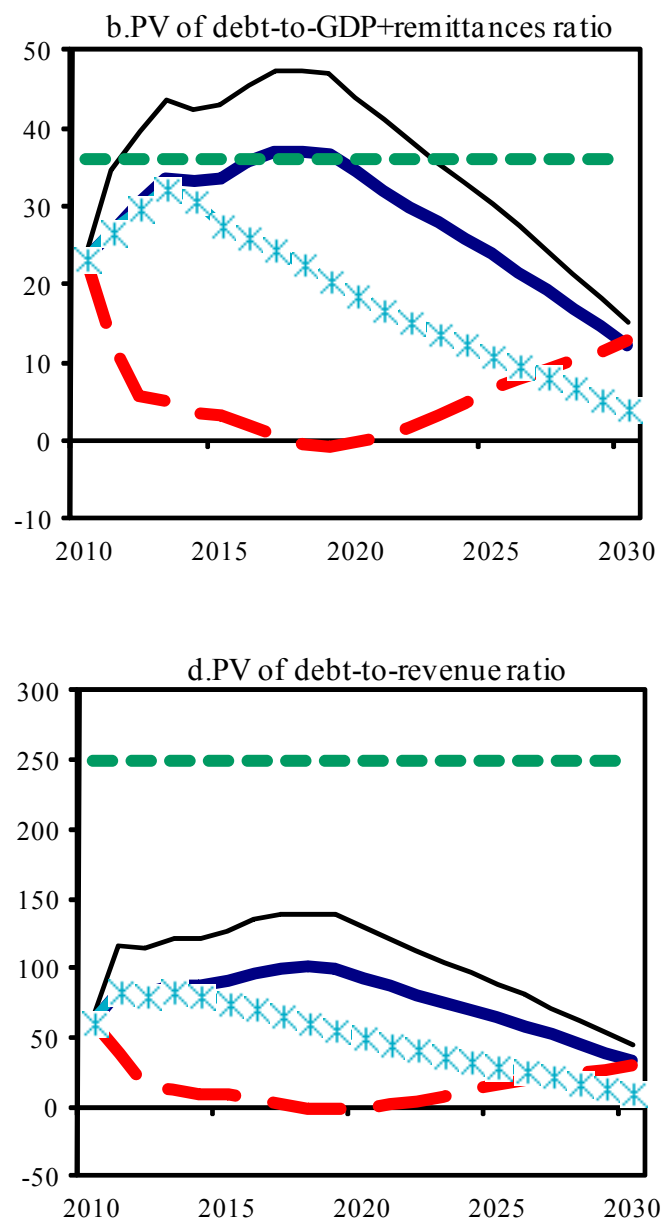

Sources: Country authorities; and staff estimates and projections.

$1 /$ The most extreme stress test is the test that yields the highest ratio in 2020. In figure b. it corresponds to a One-time depreciation shock; in c. to a Exports shock; in d. to a One-time depreciation shock; in e. to a Terms shock and in figure f. to a One-time depreciation shock 
Figure 4.Lesotho: Indicators of Public Debt Under Alternative Scenarios, 2010-2030 1/
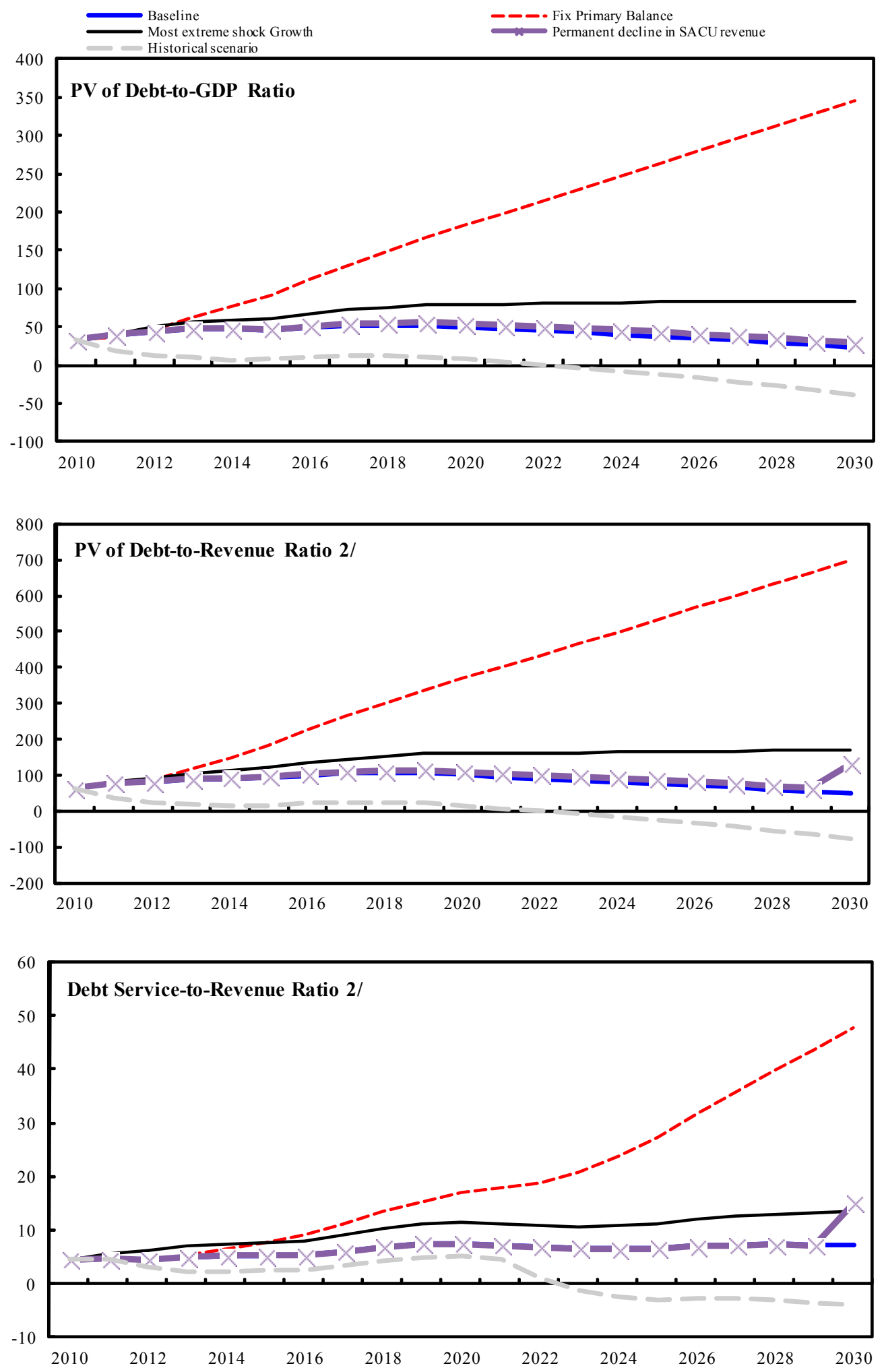

Sources: Country authorities; and staff estimates and projections.

1/ The most extreme stress test is the test that yields the highest ratio in 2020.

2/ Revenues are defined inclusive of grants. 
June 2, 2010

\section{IMF Executive Board Approves US\$61.4 Million Three-year ECF Arrangement for the Kingdom of Lesotho}

The Executive Board of the International Monetary Fund (IMF) today approved an amount equivalent to SDR 41.88 million (about US\$61.4 million) three-year arrangement for the Kingdom of Lesotho under the Extended Credit Facility (ECF). The arrangement will support the authorities' medium-term adjustment program and help reduce balance of payments risks.

Lesotho has achieved several years of macroeconomic stability, but economic performance deteriorated in 2009 as economic growth slowed due to reduced demand for its exports of textiles and diamonds, and a significant decline in workers' remittances from South Africa. The authorities' economic program focuses on fiscal consolidation and structural reforms to restore macroeconomic stability, encourage economic growth and reduce poverty.

Following the Board's decision, a sum of an amount equivalent to SDR 7.80 million (about US\$11.4 million) is available for immediate disbursement. The three-year ECF arrangement represents 120 percent of Lesotho's SDR 34.90 million IMF quota. Lesotho joined the Fund in July 1968.

Following the Executive Board's discussion of Lesotho, Mr. Naoyuki Shinohara, Deputy Managing Director and Acting Chair, made the following statement:

"The global economic crisis has had an adverse impact on Lesotho. Demand for exports has fallen, economic growth has declined, and the projected sharp reduction in Southern African Customs Union (SACU) revenues is likely to result in a significant deterioration in both the fiscal and external deficits. Strong adjustment measures and an acceleration of structural reforms are needed to restore fiscal and external sustainability, and provide the foundation for sustained broad-based growth and poverty reduction. The ambitious economic program for 2010-13, supported by the Fund's Extended Credit Facility, demonstrates the authorities' commitment to tackling these challenges. 
“The authorities' medium-term economic program appropriately focuses on fiscal consolidation. The prudent fiscal stance of the 2010/11 budget is an important first step as it seeks to contain growth in recurrent expenditure, which has risen sharply in recent years, while protecting social spending on vulnerable groups. Spending efficiency will be enhanced by further strengthening of public financial management and improving the quality of capital spending, while tax administration reform will help mobilize more non-SACU revenues. A cautious external borrowing strategy that focuses on grants and concessional loans and a strengthening of debt management capacity will be critical for debt sustainability.

"The authorities have committed to accelerating structural reforms to support economic growth and underpin fiscal consolidation. These reforms are aimed at improving the business climate and boosting productivity and external competitiveness to stimulate private sector-led growth and foster economic diversification.

“Lesotho's financial sector has remained resilient to the global crisis, and planned regulatory reforms will strengthen the sector and facilitate financial deepening. Key measures include amending the Financial Institutions Act to strengthen the supervisory role of the central bank over non-bank financial institutions, and measures to deepen the securities market and increase access to financial services, especially in the rural areas," Mr Shinohara added. 


\section{ANNEX}

\section{Recent Economic Developments}

After several years of economic stability, Lesotho's economic performance deteriorated in 2009. Economic growth slowed due to reduced export demand, while persistent increases in public expenditure in the past three years have led to a deterioration of the fiscal balance. The fiscal balance shifted into deficit in 2009/10 after five consecutive years of surpluses, and the external current account also shifted into deficit, reflecting lower export earnings and income receipts.

The global crisis has resulted in a significant decline in revenues from the Southern African Customs Union (SACU), which account for around 60 percent of the country's tax revenue. SACU revenues are projected to decline by some 23 percent of GDP during 2010/112011/12, mainly due to lower imports by South Africa. As a result, Lesotho's fiscal and external balances will deteriorate significantly, and remain vulnerable to downside risks. While SACU revenues are projected to recover somewhat, the authorities expect that these revenues will stabilize at levels well below trend.

\section{Program Summary}

The authorities' comprehensive medium-term economic program aims to respond to the impact of the reduction in SACU revenues through fiscal adjustments and structural reforms to restore fiscal and external sustainability, promote sustainable growth, a stronger financial sector and poverty reduction.

Key reform objectives include:

- Containing expenditures to levels consistent with sustainable revenue flows, while safeguarding social spending for the poor and vulnerable groups;

- Further strengthening of public expenditure and financial management;

- Improving the business climate to facilitate private sector development;

- Strengthening the regulatory framework for the financial sector and deepening financial intermediation. 
Table 1. Lesotho: Selected Economic and Financial Indicators, 2006-2015 1/

\begin{tabular}{|c|c|c|c|c|c|c|c|c|c|c|}
\hline & \multirow[b]{2}{*}{2006} & \multicolumn{3}{|c|}{ Est. } & \multicolumn{3}{|c|}{ Projections } & \multirow[b]{2}{*}{2013} & \multirow[b]{2}{*}{2014} & \multirow[b]{2}{*}{2015} \\
\hline & & 2007 & $2008^{-}$ & 2009 & 2010 & 2011 & 2012 & & & \\
\hline \multicolumn{11}{|c|}{ (Annual percentage change, unless otherwise indicated) } \\
\hline \multicolumn{11}{|l|}{ National income and prices } \\
\hline Real GDP & 6.5 & 2.4 & 4.5 & 0.9 & 5.6 & 3.3 & 5.3 & 3.0 & 5.0 & 12.1 \\
\hline Consumer price index (period average) & 6.1 & 8.0 & 10.7 & 7.2 & 6.3 & 6.0 & 5.7 & 5.0 & 5.0 & 5.0 \\
\hline Consumer price index (end of period) & 6.4 & 10.5 & 0.6 & 4.2 & 5.8 & 5.6 & 5.5 & 4.6 & 4.6 & 5.0 \\
\hline GDP (millions of maloti) & 9,587 & 11,125 & 13,185 & 13,756 & 15,402 & 16,914 & 18,770 & 20,559 & 22,529 & 26,184 \\
\hline GNP (millions of maloti) & 12,162 & 14,125 & 17,326 & 17,396 & 18,964 & 20,991 & 23,070 & 25,283 & 27,742 & 31,864 \\
\hline \multicolumn{11}{|l|}{ External sector } \\
\hline Exports, f.o.b. 2/ & 7.5 & 14.9 & 8.2 & -15.0 & 4.9 & 5.4 & 3.3 & 10.0 & 0.1 & 3.2 \\
\hline Imports, f.o.b. $2 /$ & 4.6 & 17.3 & 5.2 & -1.8 & 11.9 & 0.1 & 5.4 & -2.2 & 1.1 & 10.8 \\
\hline Nominal effective exchange rate $3 /$ & -3.4 & 0.6 & -10.3 & $\ldots$ & $\ldots$ & $\ldots$ & $\ldots$ & $\ldots$ & $\ldots$ & $\ldots$ \\
\hline Real effective exchange rate $3 /$ & -2.0 & 3.2 & -7.1 & $\ldots$ & $\ldots$ & $\ldots$ & $\ldots$ & $\ldots$ & $\ldots$ & $\ldots$ \\
\hline \multicolumn{11}{|l|}{ Money and credit } \\
\hline Net foreign assets $4 /$ & 73.2 & 66.0 & 66.6 & -8.1 & 3.2 & -2.5 & $\ldots$ & $\ldots$ & $\ldots$ & $\ldots$ \\
\hline Net domestic assets $4 /$ & -37.9 & -49.7 & -46.9 & 25.8 & 18.5 & 17.5 & $\ldots$ & $\ldots$ & $\ldots$ & $\ldots$ \\
\hline Credit to the government & -22.7 & -52.0 & -15.9 & -0.5 & 3.3 & 25.6 & $\ldots$ & $\ldots$ & $\ldots$ & $\ldots$ \\
\hline Credit to the rest of the economy & 4.3 & 9.4 & 6.2 & 7.2 & 4.8 & 4.2 & $\ldots$ & $\ldots$ & $\ldots$ & $\ldots$ \\
\hline Broad money & 35.3 & 16.4 & 19.7 & 17.7 & 21.7 & 15.0 & $\ldots$ & $\ldots$ & $\ldots$ & $\ldots$ \\
\hline Velocity (GNP/average broad money) & 4.0 & 3.7 & 3.9 & 3.3 & 3.0 & 2.8 & $\ldots$ & $\ldots$ & $\ldots$ & $\ldots$ \\
\hline Interest rate $5 /$ & 6.9 & 7.8 & 9.8 & 10.8 & $\ldots$ & $\ldots$ & $\ldots$ & $\ldots$ & $\ldots$ & $\ldots$ \\
\hline \multicolumn{11}{|c|}{ (In percent of GDP; unless otherwise indicated) } \\
\hline Investment & 24.8 & 27.8 & 30.4 & 33.2 & 39.3 & 43.4 & 41.5 & 37.1 & 34.8 & 33.4 \\
\hline Public & 7.6 & 10.4 & 13.0 & 15.7 & 21.8 & 25.9 & 23.7 & 18.0 & 14.7 & 13.3 \\
\hline Private 6/ & 17.2 & 17.4 & 17.4 & 17.5 & 17.5 & 7.5 & 17.8 & 19.1 & 20.1 & 20.1 \\
\hline Gross national savings (including remittances) & 29.4 & 41.8 & 39.9 & 32.9 & 17.3 & 20.9 & 22.7 & 28.2 & 27.2 & 21.4 \\
\hline Public $7 /$ & 21.1 & 27.8 & 23.2 & 17.8 & 8.5 & 7.6 & 14.3 & 14.4 & 13.2 & 13.9 \\
\hline Private & 8.3 & 14.0 & 16.7 & 15.1 & 8.8 & 13.3 & 8.4 & 13.8 & 14.0 & 7.5 \\
\hline \multicolumn{11}{|l|}{ Government budget $v$} \\
\hline Revenue and grants & 65.0 & 62.1 & 66.2 & 66.0 & 48.5 & 49.5 & 56.0 & 52.0 & 50.1 & 50.1 \\
\hline Grants & 0.9 & 1.5 & 17 & 4.4 & 7.6 & 10.9 & 8.2 & 5.3 & 3.5 & 3.5 \\
\hline Total expenditure and net lending & 48.7 & 53.3 & 64.6 & 68.7 & 67.5 & 67.5 & 62.7 & 54.3 & 51.0 & 48.8 \\
\hline Overall balance (excluding grants) & 15.3 & 7.2 & -0.1 & -7.1 & -26.6 & -28.8 & -14.9 & -7.7 & -4.3 & -2.2 \\
\hline Overall balance (including grants) & 16.2 & 8.7 & 16 & -2.7 & -19.0 & -17.9 & -6.8 & -2.4 & -0.8 & 1.3 \\
\hline Overall balance (excluding M etolong, after grants) & $\ldots$ & $\ldots$ & $\ldots$ & $\ldots$ & -15.3 & -14.2 & -3.1 & -1.8 & -0.4 & 1.7 \\
\hline Non-SACU overall balance (including grants) & -23.3 & -26.5 & -35.2 & -37.4 & -32.7 & -29.3 & -27.3 & -21.7 & -20.2 & -18.1 \\
\hline Primary balance & 16.2 & 11.2 & 2.5 & -1.9 & -18.1 & -16.9 & -5.5 & -1.0 & 0.5 & 2.5 \\
\hline Government debt $v$ & 54.1 & 56.7 & 52.1 & 40.2 & 45.2 & 49.8 & 53.1 & 55.3 & 54.4 & 52.4 \\
\hline Domestic debt & 7.0 & 5.8 & 4.9 & 3.9 & 5.1 & 6.1 & 6.4 & 5.8 & 5.3 & 3.9 \\
\hline External debt & 47.0 & 50.9 & 47.2 & 36.3 & 40.1 & 43.7 & 46.8 & 49.5 & 49.1 & 48.5 \\
\hline External debt-service ratio 8/ & 5.6 & 10.5 & 3.9 & 4.3 & 4.4 & 4.5 & 4.7 & 4.8 & 4.6 & 4.5 \\
\hline \multicolumn{11}{|l|}{ NVP of external debt } \\
\hline in percent of GDP & $\ldots$ & $\ldots$ & $\ldots$ & 25.5 & 28.3 & 33.0 & 37.4 & 41.3 & 41.3 & 42.2 \\
\hline in percent of GDP plus remittances & & & $\ldots$ & 20.6 & 23.3 & 26.9 & 30.6 & 33.7 & 33.2 & 33.7 \\
\hline in percent of exports of goods and services & $\ldots$ & $\ldots$ & $\ldots$ & 51.2 & 59.6 & 68.9 & 80.0 & 84.5 & 88.2 & 96.9 \\
\hline \multicolumn{11}{|l|}{ Balance of payments } \\
\hline Current acco unt balance (excl. official transfers) & -21.6 & -25.7 & -22.4 & -32.3 & -39.1 & -33.7 & -35.6 & -26.8 & -25.4 & -29.7 \\
\hline Current account balance (incl. official transfers) & 4.7 & 14.0 & 9.5 & -0.3 & -22.0 & -22.5 & -18.8 & -8.9 & -7.6 & -12.0 \\
\hline Current acco unt balance (incl. official transfers, excluding M etolong) & $\ldots$ & $\ldots$ & $\ldots$ & $\ldots$ & -20.2 & -19.8 & -15.9 & -7.4 & -6.5 & -11.1 \\
\hline \multicolumn{11}{|l|}{ Gross official reserves (end of period) } \\
\hline Millions of U.S. do llars & 664 & 946 & 923 & 1,131 & 854 & 651 & 639 & 701 & 740 & 776 \\
\hline Months of imports of goods and services & 5.4 & 6.6 & 6.2 & 7.7 & 5.2 & 4.0 & 3.7 & 4.1 & 4.3 & 4.5 \\
\hline Months of imports of goods and services excl. LHWP & 5.5 & 6.7 & 6.2 & 7.7 & 5.2 & 4.0 & 3.7 & 4.2 & 4.4 & 4.9 \\
\hline Ratio of reserves/M2 & 1.3 & 1.6 & 1.8 & 1.5 & 1.1 & 0.8 & 0.7 & 0.7 & 0.7 & 0.6 \\
\hline Ratio of reserves/M 1 & 1.7 & 2.2 & 2.2 & 2.0 & 1.5 & 1.0 & 1.0 & 1.0 & 0.9 & 0.9 \\
\hline
\end{tabular}

Sources: Lesotho authorities; and IMF staff estimates and projections.

$\checkmark$ Fiscal data are reported on a fiscal year basis; the fiscal year begins in A pril.

2/ U.S. dollars.

3/ Based on partner-country data, new trade weights from 2004; a minus sign indicates a depreciation.

4 / Change in percent of broad money at the beginning of the period.

$5 /$ The average effective rate on three-month treasury bills.

6/ Includes changes in inventories and gross fixed capital formation by the Lesotho Highlands Development Authority (LHDA).

7/ For 2008-10, government current expenditure has been reduced by the actual and prospective transfer to the pension fund.

8/ Percent of exports of goods and nonfactor services. 


\section{Statement by Mr. Majoro on Kingdom of Lesotho June 2, 2010}

We thank staff for their cooperation and constructive policy dialogue with the authorities during the negotiation of the ECF program. We also thank the Executive Board and Management for their continued support. The authorities are in general agreement with the thrust of the staff reports.

The recent economic crisis has presented a serious setback to the significant progress that Lesotho had achieved in attaining macroeconomic stability. It has led to a slowdown in economic growth as well as a very large and unprecedented decline in SACU revenues, with significant adverse implications on fiscal and external stability. The authorities fully acknowledge that, in this challenging environment, preserving stability would require a significantly large fiscal adjustment. However, care should be taken to safeguard the foundations for economic growth and to protect the poor and vulnerable. In this regard, the authorities have adopted a program of economic and financial policies aimed at bringing the economy back to a sustainable and high growth path and are requesting a Fund ECF program support to reinforce their policies. The ECF is an appropriate instrument given that the SACU shock is not considered a short-term phenomenon but is expected to have protracted effects on the economy. The ECF would therefore support the medium-term adjustment needed to bring the economy back to stability and growth.

\section{Macroeconomic economic developments and outlook}

Over the past years fiscal and external stability had been facilitated by the prudent use of fiscal surpluses to reduce public debt through early repayment of non-concessional debt and to build a comfortable cushion of external reserves. Recently however, economic performance has been adversely affected through reductions in textile exports as a result of lower external demand--especially in the US, as well as the fall in mining output due to unfavorable developments in international markets for rough diamonds. Economic growth was estimated to decline to 0.9 percent in 2009 compared with 4.5 percent in 2008. Inflation decelerated to around 4 percent at the end of 2009, in line with regional developments.

Fiscal and external positions were also negatively impacted through the large and unprecedented decline in SACU revenues as a result of the slowdown in private consumption in South Africa. Following five consecutive years of surpluses, the fiscal balance deteriorated into a deficit equivalent to 2.7 percent of GDP as a result of the large drop in SACU revenues. The drop in SACU revenues coupled with reduced remittances from South Africa also led to the shift in the current account balance from surplus, which averaged more than 9 percent of GDP over the previous 3 years, into a deficit of 0.3 percent of GDP in 2009. However, the level of international reserves rose to 7.7 months of import coverage at the end of 2009 as capital flows more than offset the deterioration in the current account balance.

SACU revenues are expected to decline sharply by a total of 23 percent of GDP during 2010/11-2011/12, due to the contraction in the South African economy. This is expected to have a very large adverse impact on Lesotho's fiscal and external positions. Although SACU 
revenues are expected to recover somewhat with economic recovery, they are expected to stabilize at levels below trend as the recovery in South Africa is not expected to be sufficient to restore SACU revenues to previous levels.

\section{Macroeconomic policies}

The authorities' macroeconomic policies are intended to adjust the economy in light of lower SACU revenues and restore macro-economic stability while limiting the adverse impact of fiscal consolidation on the poor and vulnerable. The policies are consistent with Lesotho's Interim National Development Framework (INDF) which outlines the country's mediumterm development objectives for growth and poverty reduction. The program focuses on restoring fiscal and external sustainability, achieving broad-based growth for sustained poverty reduction and strengthening the financial sector and enhancing access to financial services.

\section{Fiscal policies}

Starting with the 2010/11 budget, the authorities will be taking concrete fiscal consolidation steps geared towards regaining macroeconomic stability. They plan to take measures to significantly reduce expenditures and enhance domestic revenue collection to levels that are consistent with medium-term fiscal sustainability while protecting critical investment and social spending. To this end, they will contain growth in recurrent spending by restraining the wage bill, rationalizing spending on goods and services as well as putting in place measures to enhance the efficiency of spending. Containing the wage bill will entail the restriction of new recruitment to high-priority areas, freezing the number of existing positions, eliminating those that have remained vacant over an extended period of time and controlling general wage increases during the program period. A comprehensive review of the public service to identify non-core functions of the government and facilitate the reduction of non-essential operations will also be carried out.

With regard to capital spending, the reduction in recurrent spending is expected to create some fiscal space to protect some essential public investment. To improve the effectiveness and efficiency of capital expenditure, the authorities have resuscitated the Project Appraisal Committee, which is responsible for the assessment and recommendation of new projects to ensure that only projects that meet the criteria for enhancing broad-based economic growth, poverty reduction, and attaining the MDGs are included in the budget. The authorities also intend to conduct a comprehensive review of on-going capital projects with a view to assessing their desirability and making recommendations to Cabinet on which projects are no longer needed or effective. The results of this review will be submitted to Cabinet by September 2010.

On the revenue side the authorities are cognizant of the limited scope they have in increasing domestic revenues given that, at 27 percent, they are already high relative to GDP. However, they will continue to make efforts to strengthen tax revenues by improving tax administration 
and compliance. They will also undertake a review of nontax revenues to establish areas that can potentially contribute more revenue.

The authorities will also continue building on the progress made in reforming the Public Financial Management (PFM) system. This will include addressing the deficiencies in the functioning of the Integrated Financial Management Information System (IFMIS), guided by the recommendations of the recent IMF Technical Assistance mission on PFM, in order to enhance its effectiveness. Improving the functionality of the IFMIS will include actions to strengthen commitment and internal controls. The authorities will also address the issue of domestic payments arrears that arose due to some IFMIS problems and clear them. It must be noted that the arrears did not stem from any shortage of funds but from technical problems with the system.

Even with the above measures in place, the decline in SACU revenues is projected to widen the fiscal deficit (excluding outlays for the Metolong dam) to an average of 15 percent of GDP in 2010/11-2011/12. However, it must be noted that this would have resulted from a very significant fiscal consolidation effort and without these measures the deficit for the 2010/11 fiscal year would be as high as 23 percent of GDP. The government plans to finance the deficit mainly by drawing down its deposits at the Central Bank of Lesotho (CBL) and by issuing domestic bonds. The drawdown of government deposits will be executed cautiously, bearing in mind the need to preserve a favorable balance of payments position and to maintain the exchange rate peg. In addition, budgetary grants and concessional loans have been secured from some development partners. The authorities expect to attain a sustainable fiscal position over the medium term.

Prudent debt management remains high on the authorities' list of priorities and they will continue to strengthen their debt management capacity and as much as possible limit new debt to concessional borrowing. However, non-concessional borrowing will be considered, in the absence of adequate concessional funding, in cases where the government projects are considered significantly important for supporting growth. For example, the authorities have allowed non-concessional loans for the construction of the Metolong Dam and the phase II of the Lesotho Highlands Water Project because of the considerable contribution the two projects are expected to make to economic growth and government revenues in the medium to long term.

The authorities are also working on strengthening governance and public accountability. To this end they are preparing a Statement Affairs as at end March 2008 to facilitate the preparation and audit of financial accounts for financial years starting with 2008/09. The legal framework for budget execution is also being developed and the draft Public Financial Management Bill will be submitted for cabinet consideration in June 2010. 


\section{Strengthening the financial sector and enhancing access to financial services}

A strong financial sector and financial market is important to support the financing of economic activity and development. Thus the authorities continue to place great emphasis on strengthening financial institutions and deepening the money and capital markets. To this end the authorities are developing a domestic bond market to help deepen the securities market, and provide government with an alternative source of financing for priority projects. The first bond issue is expected by December 2010 .

The authorities will continue their efforts to improve prudential supervision and regulation of the financial sector. To this end the Financial Institutions Act (FIA) is being reviewed to provide for the strengthening of prudential framework for supervision of nonbank financial institutions and the prevention and resolution of future emergence of illegal financial schemes. Following parliamentary approval of the Anti-Money Laundering Act in 2008, the Financial Intelligence Unit has been established with the assistance of development partners. To operationalize the unit, regulations for the mandate and broader AML regime are being prepared with the technical assistance from the US treasury.

The government, with the support of $\mathrm{MCC}$ and the International Fund for Agricultural Development (IFAD), is pursing efforts to improve access to financial services for rural areas. In this regard, the provision of micro-lending products to individuals through the Postbank has started. The plan is to expand this service to small and medium-size enterprises in the near future. In this regard, the government is in the process of designing a loan guarantee scheme, building on experience with such schemes in the past.

\section{External stability and growth policies}

Achieving external sustainability will be facilitated by fiscal consolidation, implementation of key structural reforms to enhance productivity growth in the export sector and improvement of infrastructure. In using reserves to finance the deficit, the authorities will remain vigilant to maintain a sufficient level of reserves to support the exchange rate peg and avert balance of payments problems.

The authorities continue to give precedence to the acceleration of shared and sustainable economic growth to reduce poverty and inequality. In the medium-term, growth will be supported by the implementation of macroeconomic and structural reforms aimed at increasing competitiveness and achieving diversification. An expansion in private sector activities, complemented by public investment in infrastructure, including the construction of the Metolong Dam, the start up of the second phase of the Lesotho Highlands Water Project (LHWP II) and development of further mining opportunities will help to underpin growth. In this regard, the authorities are pursuing efforts, with the support of World Bank, MCC and IFAD to improve the investment climate.

The MCC's private sector component, the World Bank's ongoing Private Sector Competitiveness and Economic Diversification project and IFAD's rural Financial 
Intermediation Program are expected to help promote investment by, among others, helping address the legal and regulatory constraints to business as well as improving private sector access to financial services. The authorities also plan to improve the legal framework for doing business through the Companies and the Industrial licensing bills that will be submitted to Parliament by September 2010.

\section{Conclusion}

The authorities are committed to the successful implementation of the program and are prepared to take additional measures in this regard should the need arise. The continued support of the Fund and the international community to help them achieve their economic development goals is greatly appreciated. 